\title{
CLUSTERING IN BLOCK MARKOV CHAINS
}

\author{
By Jaron Sanders ${ }^{*} \dagger$ Alexandre Proutière* And Se-Young Yun ${ }^{\ddagger}$ \\ KTH Royal Institute of Technology*, Sweden \\ Delft University of Technology ${ }^{\dagger}$, The Netherlands \\ Korea Advanced Institute of Science and Technology $y^{\ddagger}$, South Korea
}

\begin{abstract}
This paper considers cluster detection in Block Markov Chains (BMCs). These Markov chains are characterized by a block structure in their transition matrix. More precisely, the $n$ possible states are divided into a finite number of $K$ groups or clusters, such that states in the same cluster exhibit the same transition rates to other states. One observes a trajectory of the Markov chain, and the objective is to recover, from this observation only, the (initially unknown) clusters. In this paper we devise a clustering procedure that accurately, efficiently, and provably detects the clusters. We first derive a fundamental information-theoretical lower bound on the detection error rate satisfied under any clustering algorithm. This bound identifies the parameters of the BMC, and trajectory lengths, for which it is possible to accurately detect the clusters. We next develop two clustering algorithms that can together accurately recover the cluster structure from the shortest possible trajectories, whenever the parameters allow detection. These algorithms thus reach the fundamental detectability limit, and are optimal in that sense.
\end{abstract}

1. Introduction. The ability to accurately discover all hidden relations between items that share similarities is of paramount importance to a wide range of disciplines. Clustering algorithms in particular are employed throughout social sciences, biology, computer science, economics, and physics. The reason these techniques have become prevalent is that once clusters of similar items have been identified, any subsequent analysis or optimization procedure benefits from a powerful reduction in dimensionality.

The canonical Stochastic Block Model (SBM), originally introduced in [1], has become the benchmark to investigate the performance of cluster detection algorithms. This model generates random graphs that contain groups of similar vertices. Vertices within the same group are similar in that they share the same average edge densities to the other vertices. More precisely, if the set of $n$ vertices $\mathcal{V}$ is for example partitioned into two groups $\mathcal{V}_{1}$ and $\mathcal{V}_{2}$, an edge is drawn between two vertices $x, y \in \mathcal{V}$ with probability

MSC 2010 subject classifications: Primary 62H30, 60J10, 60J20

Keywords and phrases: clustering, Markov chains, mixing times, community detection, change of measure, asymptotic analysis, information theory

imsart-aos ver. 2014/10/16 file: JS_AP_SY__CBMC__arXiv.tex date: July 31, 2019 
$p \in(0,1)$ if they belong to the same group, and with probability $q \in(0,1)$, $p \neq q$, if they belong to different groups. Edges are drawn independently of all other edges. Within the context of the SBM and its generalizations, the problem of cluster detection is to infer the clusters from observations of a realization of the random graph with the aforementioned structure.

This paper deviates by considering the problem of cluster detection when the observation is instead the sample path of a Markov chain over the set of vertices. Specifically, we introduce the Block Markov Chain (BMC), which is a Markov chain characterized by a block structure in its transition matrix. States that are in the same cluster are similar in the sense that they have the same transition rates. The goal is to detect the clusters from an observed sample path $X_{0}, X_{1}, \ldots, X_{T}$ of the Markov chain (Figure 1). This new clustering problem is mathematically more challenging because consecutive samples of the random walk are not independent: besides noise, there is bias in a sample path. Intuitively though there is hope for accurate cluster detection if the Markov chain can get close to stationarity within $T$ steps. Indeed, as we will show, the mixing time [2] of the BMC plays a crucial role in the detectability of the clusters.

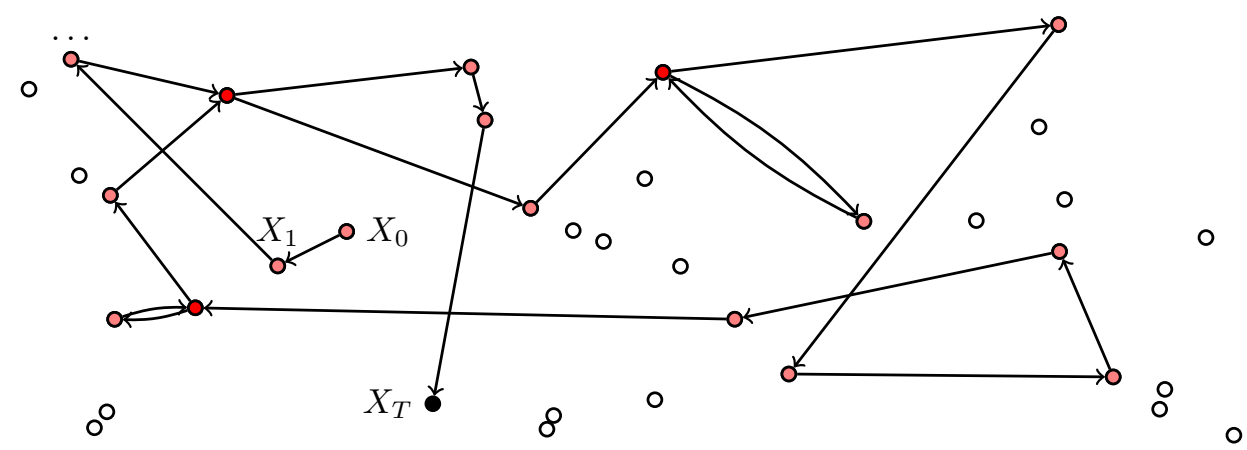

Fig 1: The goal of this paper is to infer the hidden cluster structure underlying a Markov chain $\left\{X_{t}\right\}_{t \geq 0}$, from one observation of a sample path $X_{0}, X_{1}, \ldots, X_{T}$ of length $T$.

Clustering in BMCs is motivated by Reinforcement Learning (RL) problems [3] with large state spaces. These problems have received substantial attention recently due to the wide spectrum of their applications in robotics, games, medicine, finance, etc. RL problems are concerned with the control of dynamical systems modeled as Markov chains whose transition kernels are initially unknown. The objective is to identify an optimal control policy as early as possible by observing the trajectory of a Markov chain generated under some known policy. The time it takes to learn efficient policies using

imsart-aos ver. 2014/10/16 file: JS_AP_SY_CBMC__arXiv.tex date: July 31, 2019 
standard algorithms such as Q-learning dramatically increases with the number of possible states, so that these algorithms become useless when the state space is prohibitively large. In most practical problems however, different states may yield similar reward and exhibit similar transition probabilities to other states, i.e., states can be grouped into clusters. In this scenario it becomes critical to learn and leverage this structure in order to speed up the learning process. In this paper we consider uncontrolled Markov chains, and we aim to identify clusters of states as quickly as possible. In the future we hope to extend the techniques developed here for an uncontrolled BMC to the more general case of controlled Markov chains, and hence to devise reinforcement learning algorithms that will efficiently exploit an underlying cluster structure. The idea of clustering states in reinforcement learning to speed up the learning process has been investigated in [4] and [5], but no theoretical guarantees were provided in these early papers.

This paper answers two important questions for the problem of cluster detection on BMCs. First, we derive a fundamental information-theoretical clustering error lower bound. The latter allows us to identify the parameters of the BMC and the sample path lengths $T$ for which it is theoretically impossible to accurately detect the underlying cluster structure. Second, we develop two clustering algorithms that when combined, are able to accurately detect the underlying cluster structure from the shortest possible sample paths, whenever the parameters of the BMC allow detection, and that provably work as $n \rightarrow \infty$. These algorithms thus reach the fundamental detectability limit, and are optimal in that sense.

1.1. Related work. Clustering in the SBM and the BMCs may be seen as similar problems: the objective in both cases is to infer the cluster structure from random observations made on the relationships between pairs of vertices. However, the way these observations are gathered differ significantly in the SBM and the BMCs. In the SBM, these observations are independent random variables, which allows the use of theoretical developments in random matrices with independent entries. In the BMCs on the contrary, observations are successive states of a Markov chain and hence are not independent. Furthermore, observed edges in the SBM are scattered and undirected, whereas in a BMC, the observed path is a concatenation of directed edges. Generally the probabilities to move from state $x$ to state $y$ and from $y$ to $x$ are different. Finally, the sparsity of the observations in the BMC is controlled by the length $T$ of the observed sample path, while it is hard-coded in the SBM. For all these reasons, it is difficult to quantitatively compare or relate the recovery rates in the two models. Nevertheless, techniques as those used

imsart-aos ver. 2014/10/16 file: JS_AP_SY_CBMC__arXiv.tex date: July 31, 2019 
in the SBM can be exploited in the analysis of the BMC if they are properly extended to handle the differences between the two models. For this reason, we now provide a brief survey of the techniques and results available for the SBM.

Significant advances have been made on cluster recovery within the context of the SBM and its generalizations. We defer the reader to [6] for an extensive overview. Substantial focus has in particular been on characterizing the set of parameters for which some recovery objectives can be met.

In the sparse regime, i.e., when the average degree of vertices is $O(1)$, necessary and sufficient conditions on the parameters have been identified under which it is possible to extract clusters that are positively correlated with the true clusters [7-9]. More precisely, for example if $p=a / n$ and $q=b / n$ and in the case of two clusters of equal sizes, it was conjectured in [7] that $a-b \geq \sqrt{2(a+b)}$ is a necessary and sufficient condition for the existence of algorithms that can detect the clusters (in the sense that they perform better than a random assignment of items to clusters). This result was established in [9] (necessary condition) and in [8] (sufficient condition).

In the dense regime, i.e., when the average degree is $\omega(1)$, it is possible to devise algorithms under which the proportion of misclassified vertices vanishes as the size of the graph grows large [10]. In this case, one may actually characterize the minimal asymptotic (as $n$ grows large) classification error, and develop clustering algorithms achieving this fundamental limit [11]. We may further establish conditions under which asymptotic exact cluster recovery is possible [11-18].

This paper draws considerable inspiration from [10-12]. Over the course of these papers, the authors consider the problem of clustering in the Labeled Stochastic Block Model (LSBM), which is a generalization of the SBM. They identify the set of LSBM-parameters for which the clusters can be detected using change-of-measure arguments, and develop algorithms based on spectral methods that achieve this fundamental performance limit. Our contributions in this paper include the extension of the approaches to the context of Markov chains. This required us in particular to design novel changes-of-measure, carefully incorporate the effect of mixing, deal with new and non-convex log-likelihood functions, and widen the applicability of spectral methods to random matrices with bias. Note that we restrict the analysis in this paper to the case that the number of clusters $K$ is known. This reduces the complexity of the analysis. Based on the findings in [10-12] however, we are confident that this assumption can be relaxed in future work.

imsart-aos ver. 2014/10/16 file: JS_AP_SY__CBMC_-arXiv.tex date: July 31, 2019 
1.2. Methodology. Similar to the extensive efforts for the SBM, we will first identify parameters of the BMC for which it is theoretically possible to detect the clusters. To this aim, we use techniques from information theory to derive a lower bound on the number of misclassified states that holds for any classification algorithm. This relies on a powerful change-of-measure argument, originally explored in [19] in the context of online stochastic optimization. First, we relate the probability of misclassifying a state in the BMC to a log-likelihood ratio that the sample path was generated by a perturbed Markov chain instead. Then, given any BMC, we show how to construct a perturbed Markov chain that assigns a nonzero probability to the event that all clustering algorithms misclassify at least one particular state. Finally, we maximize over all possible perturbations to get the best possible lower bound that holds for any algorithm.

We will further provide a clustering algorithm that achieves this fundamental limit. Specifically, the algorithm consists of two steps. The first step consists in applying a classical Spectral Clustering Algorithm. This algorithm essentially creates a rank- $K$ approximation of a random matrix corresponding to the empirical transition rates between any pair of states, and then uses a $K$-means algorithm [20] to cluster all states. We show that this first step clusters the majority of states roughly correctly. Next, we introduce the Cluster Improvement Algorithm. This algorithm uses the rough structure learned from the Spectral Clustering Algorithm, together with the sample path, to move each individual state into the cluster the state most likely belongs to. This is achieved through a recursive, local maximization of a log-likelihood ratio.

The key difference between clustering in SBMs and clustering in BMCs is that instead of observing (the edges of) a random graph, we here try to infer the cluster structure from an as short as possible sample path of the Markov chain. This necessitates a careful analysis of the mixing time of the Markov chain [2], for which we use a rate of convergence result in terms of Dobrushin's ergodicity coefficient [21]. The observed sample path will be inherently noisy and biased by construction. The noise and bias within the sample path have to first be related to the spectrum of the random matrix recording the number of times transitions between any two states have been observed. This is done by using techniques from [22]. The spectrum of this random matrix has then to be analyzed which constitutes a major challenge. Indeed, most results investigating the spectrum of random matrices hold for matrices with independent and weakly dependent entries [23-28], or when the transition matrix of the Markov chain itself is random [29, 30]. Our random matrix has dependent entries, but by taking proof inspiration from

imsart-aos ver. 2014/10/16 file: JS_AP_SY_CBMC__arXiv.tex date: July 31, 2019 
[31], using concentration results from [32], and smartly leveraging the way it is constructed from the observed sample path and Markov property, the analysis of its spectrum can be conducted.

1.3. Overview. This paper is structured as follows. We introduce the BMC in Section 2. Section 3 provides an overview of our results and our algorithms. We assess the performance of both algorithms, i.e., we quantify their asymptotic error rates. Section 4 discusses several numerical experiments designed to test the algorithms. We subsequently prove our results by first deriving an information lower bound and developing an optimal changeof-measure in Section 5, and then by developing the Spectral Clustering Algorithm in Section 6 and the Cluster Improvement Algorithm in Section 7.

Notation. For any two sets $\mathcal{A}, \mathcal{B} \subseteq \mathcal{V} \triangleq\{1, \ldots, n\}$ we define their symmetric difference by $\mathcal{A} \triangle \mathcal{B}=\{\mathcal{A} \backslash \mathcal{B}\} \cup\{\mathcal{B} \backslash \mathcal{A}\}$. For any two numbers $a, b \in \mathbb{R}$ we introduce the shorthand notations $a \wedge b=\min \{a, b\}$ and $a \vee b=$ $\max \{a, b\}$. For any $n$-dimensional vector $x=\left(x_{1}, \ldots, x_{n}\right)^{\mathrm{T}} \in \mathbb{R}^{n}$, we define its $l_{p}$ norms by

$$
\|x\|_{p}=\left(\sum_{r=1}^{n}\left|x_{i}\right|^{p}\right)^{1 / p} \quad \text { where } \quad p \in[1, \infty) .
$$

The $n$-dimensional unit vector of which the $r$-th component equals 1 will be denoted by $e_{n, r}$, and the $n$-dimensional vector for which all elements $r \in \mathcal{A} \subseteq\{1, \ldots, n\}$ equal 1 will be denoted by $1_{\mathcal{A}}$. For any $m \times n$ matrix $A \in \mathbb{R}^{m \times n}$, we indicate its rows by $A_{r}$. for $r=1, \ldots, m$ and its columns by $A_{,, c}$ for $c=1, \ldots, n$. We also introduce the short-hand notation $A_{\mathcal{A}, \mathcal{B}}=$ $\sum_{x \in \mathcal{A}} \sum_{y \in \mathcal{B}} A_{x, y}$ for all subsets $\mathcal{A}, \mathcal{B} \subseteq \mathcal{V}$. Its Frobenius norm and spectral norm are defined by

$$
\|A\|_{\mathrm{F}}=\sqrt{\sum_{r=1}^{m} \sum_{c=1}^{n} A_{r, c}^{2}}, \quad\|A\|=\sup _{b \in \mathbb{S}^{n-1}}\left\{\|A b\|_{2}\right\},
$$

respectively. Here, $\mathbb{S}^{n-1}=\left\{x=\left(x_{1}, \ldots, x_{n}\right) \in(0,1)^{n}:\|x\|_{2}=1\right\}$ denotes the $n$-dimensional unit sphere. We define the probability simplex of dimension $n-1$ by $\Delta^{n-1}=\left\{x \in(0,1)^{n}:\|x\|_{1}=1\right\}$ as well as the set of leftstochastic matrices by $\Delta^{n \times(n-1)}=\left\{\left(\left(x_{1,1}, \ldots, x_{1, n}\right), \ldots,\left(x_{n, 1}, \ldots, x_{n, n}\right)\right) \in\right.$ $[0,1]^{n \times n}: \sum_{c=1}^{n} x_{r, c}=1$ for $\left.r=1, \ldots, n\right\}$ similarly.

In our asymptotic analyses, we write $f(n) \sim g(n)$ if $\lim _{n \rightarrow \infty} f(n) / g(n)=1$, $f(n)=o(g(n))$ if $\lim _{n \rightarrow \infty} f(n) / g(n)=0$ and $f(n)=O(g(n))$ if $\limsup _{n \rightarrow \infty}$

imsart-aos ver. 2014/10/16 file: JS_AP_SY__CBMC_-arXiv.tex date: July 31, 2019 
$f(n) / g(n)<\infty$. Whenever $\left\{X_{n}\right\}_{n=1}^{\infty}$ is a sequence of real-valued random variables and $\left\{a_{n}\right\}_{n=1}^{\infty}$ a deterministic sequence, we write

$$
\begin{gathered}
X_{n}=o_{\mathbb{P}}\left(a_{n}\right) \Leftrightarrow \mathbb{P}\left[\left|\frac{X_{n}}{a_{n}}\right| \geq \delta\right] \rightarrow 0 \forall_{\delta>0} \Leftrightarrow \forall_{\varepsilon, \delta} \exists_{N_{\varepsilon, \delta}}: \mathbb{P}\left[\left|\frac{X_{n}}{a_{n}}\right| \geq \delta\right] \leq \varepsilon \forall_{n>N_{\varepsilon, \delta}}, \\
\text { and } X_{n}=O_{\mathbb{P}}\left(a_{n}\right) \Leftrightarrow \forall_{\varepsilon} \exists_{\delta_{\varepsilon}, N_{\varepsilon}}: \mathbb{P}\left[\left|\frac{X_{n}}{a_{n}}\right| \geq \delta_{\varepsilon}\right] \leq \varepsilon \forall_{n>N_{\varepsilon}} .
\end{gathered}
$$

Similarly, $X_{n}=\Omega_{\mathbb{P}}\left(a_{n}\right)$ denotes $\forall_{\varepsilon} \exists_{\delta_{\varepsilon}, N_{\varepsilon}}: \mathbb{P}\left[\left|X_{n} / a_{n}\right| \leq \delta_{\varepsilon}\right] \leq \varepsilon \forall_{n>N_{\varepsilon}}$, and $X_{n} \asymp \mathbb{P}\left(a_{n}\right)$ means $\forall_{\varepsilon} \exists_{\delta_{\varepsilon}^{-}, \delta_{\varepsilon}^{+}, N_{\varepsilon}}: \mathbb{P}\left[\delta_{\varepsilon}^{-} \leq\left|X_{n} / a_{n}\right| \leq \delta_{\varepsilon}^{+}\right] \geq 1-\varepsilon \forall_{n>N_{\varepsilon}}$.

2. Block Markov Chains (BMCs). We assume that we have $n$ states $\mathcal{V}=\{1, \ldots, n\}$, each of which is associated to one of $K$ clusters. This means that the set of states is partitioned so that $\mathcal{V}=\cup_{k=1}^{K} \mathcal{V}_{k}$ with $\mathcal{V}_{k} \cap \mathcal{V}_{l}=\emptyset$ for all $k \neq l$. Let $\sigma(v)$ denote the cluster of a state $v \in \mathcal{V}$. We also assume that there exist constants $\alpha \in \Delta^{K-1}$ so that $\lim _{n \rightarrow \infty}\left|\mathcal{V}_{k}\right| /\left(n \alpha_{k}\right)=1$.

For any $\alpha \in \Delta^{K-1}$ and $p \in \Delta^{K \times(K-1)}$, we define the BMC $\left\{X_{t}\right\}_{t \geq 0}$ as follows. Its transition matrix $P \in \Delta^{n \times(n-1)}$ will be defined as

$$
P_{x, y} \triangleq \frac{p_{\sigma(x), \sigma(y)}}{\left|\mathcal{V}_{\sigma(y)}\right|-\mathbb{1}[\sigma(x)=\sigma(y)]} \mathbb{1}[x \neq y] \quad \text { for all } \quad x, y \in \mathcal{V} .
$$

Note that this Markov chain is not necessarily reversible. Furthermore, note that in this paper we assume that $K, \alpha, p$ are fixed, and that we study the asymptotic regime $n \rightarrow \infty$. We assume that the smallest cluster has a size linearly growing with $n$ : $\alpha_{\min } \triangleq \min _{k} \alpha_{k}>0$. Finally, since we are interested in clustering of the states, we will assume that $\exists_{1<\eta}: \max _{a, b, c}\left\{p_{b, a} / p_{c, a}\right.$, $\left.p_{a, b} / p_{a, c}\right\} \leq \eta$, which guarantees a minimum level of separability of the parameters.

2.1. Equilibrium behavior. We assume that the stochastic matrix $p$ is such that the equilibrium distribution of $\left\{X_{t}\right\}_{t \geq 0}$ exists, and we will denote it by $\Pi_{x}$ for $x \in \mathcal{V}$. By symmetry, $\Pi_{x}=\Pi_{y} \triangleq \bar{\Pi}_{k}$ for any two states $x, y \in \mathcal{V}_{k}$ for all $k=1, \ldots, K$. Consider the scaled quantity

$$
\pi_{k} \triangleq \lim _{n \rightarrow \infty} \sum_{x \in \mathcal{V}_{k}} \Pi_{x}=\lim _{n \rightarrow \infty}\left|\mathcal{V}_{k}\right| \bar{\Pi}_{k} \quad \text { for } \quad k=1, \ldots, K .
$$

Proposition 1's proof can be found in §SM2.1, and follows from the symmetries between the states within the same clusters and the specific scalings of $P$ 's elements.

Proposition 1. The quantity $\pi$ solves $\pi^{\mathrm{T}} p=\pi^{\mathrm{T}}$, and is therefore the equilibrium distribution of a Markov chain with transition matrix $p$ and state space $\Omega=\{1, \ldots, K\}$.

imsart-aos ver. 2014/10/16 file: JS_AP_SY_CBMC__arXiv.tex date: July 31, 2019 
2.2. Mixing time. Proposition 2 gives a bound on the mixing time $t_{\text {mix }} \in(0, \infty)$, which is defined by $d(t) \triangleq \sup _{x \in \mathcal{V}}\left\{d_{\mathrm{TV}}\left(P_{x,}^{t}, \Pi\right)\right\}$ and $t_{\text {mix }}(\varepsilon) \triangleq$ $\min \{t \geq 0: d(t) \leq \varepsilon\}$, where

$$
d_{\mathrm{TV}}(\mu, \nu) \triangleq \frac{1}{2} \sum_{x \in \mathcal{V}}\left|\mu_{x}-\nu_{x}\right| .
$$

The proof of Proposition 2 is deferred to §SM2.2. The result follows after bounding Dobrushin's ergodicity coefficient [21] using $P$ 's structure, and invoking a convergence rate result in terms of Dobrushin's coefficient.

Proposition 2. For any BMC with $n \geq 4 / \alpha_{\text {min }}, t_{\text {mix }}(\varepsilon) \leq-c_{\text {mix }} \ln \varepsilon$, where $c_{\text {mix }}=-1 / \ln (1-1 / 2 \eta)$.

Proposition 2 implies that the mixing times are short enough so that our results will hold irrespective of whether we assume that the Markov chain is initially in equilibrium. We will show in Section 5.4 that what is important is that the chain reaches stationarity within $T$ steps (the length of the observed trajectory), and consequentially, $T$ needs to be chosen sufficiently large with respect to $n$ to ensure that this occurs. Throughout this paper we therefore assume for simplicity that the chain is started from equilibrium. This eliminates the need of tracking higher order correction terms.

Examples. Figure 2 illustrates the structure of a BMC when there are $K=2$ groups. We find after solving the balance equations that the limiting equilibrium behavior is given by $\pi_{1}=p_{21} /\left(p_{12}+p_{21}\right)$ and $\pi_{2}=p_{12} /\left(p_{12}+p_{21}\right)$.

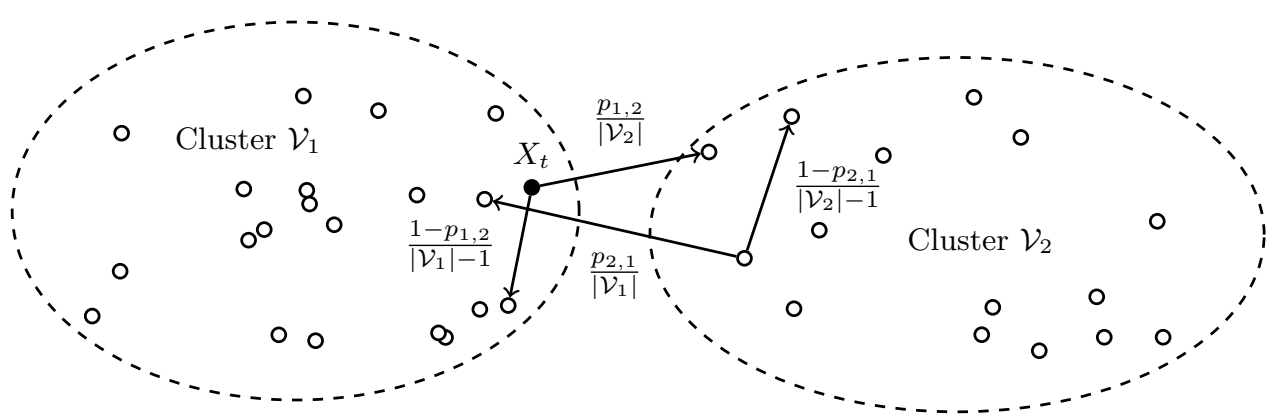

Fig 2: In the BMC with $K=2$ groups $\mathcal{V}_{1} \cup \mathcal{V}_{2}=\mathcal{V}$, whenever the Markov chain is at some state $X_{t} \in \mathcal{V}_{1}$, it will next jump with probability $p_{1,2}$ to cluster $\mathcal{V}_{2}$, and with probability $1-p_{1,2}$ to some other state in cluster $\mathcal{V}_{1}$ Similarly, if $X_{t} \in \mathcal{V}_{2}$, it would next jump to cluster $\mathcal{V}_{1}$ with probability $p_{2,1}$, or stay within its own cluster with probability $1-p_{2,1}$.

imsart-aos ver. 2014/10/16 file: JS_AP_SY__CBMC_-arXiv.tex date: July 31, 2019 
For $K=3$, we find after solving the balance equations that the limiting equilibrium behavior is given by

$$
\pi_{1}=\frac{p_{23} p_{31}+p_{21}\left(p_{31}+p_{32}\right)}{Z(p)}, \quad \pi_{2}=\frac{p_{13} p_{32}+p_{12}\left(p_{31}+p_{32}\right)}{Z(p)},
$$

$\pi_{3}=1-\pi_{1}-\pi_{2}$, with $Z(p)=\left(p_{21}+p_{23}\right)\left(p_{13}+p_{31}\right)+\left(p_{13}+p_{21}\right) p_{32}+p_{12}\left(p_{23}+\right.$ $\left.p_{31}+p_{32}\right)$. Let us also illustrate the structure of the transition matrix when $\alpha=(2 / 10,3 / 10,5 / 10)$ and $n=10$ :

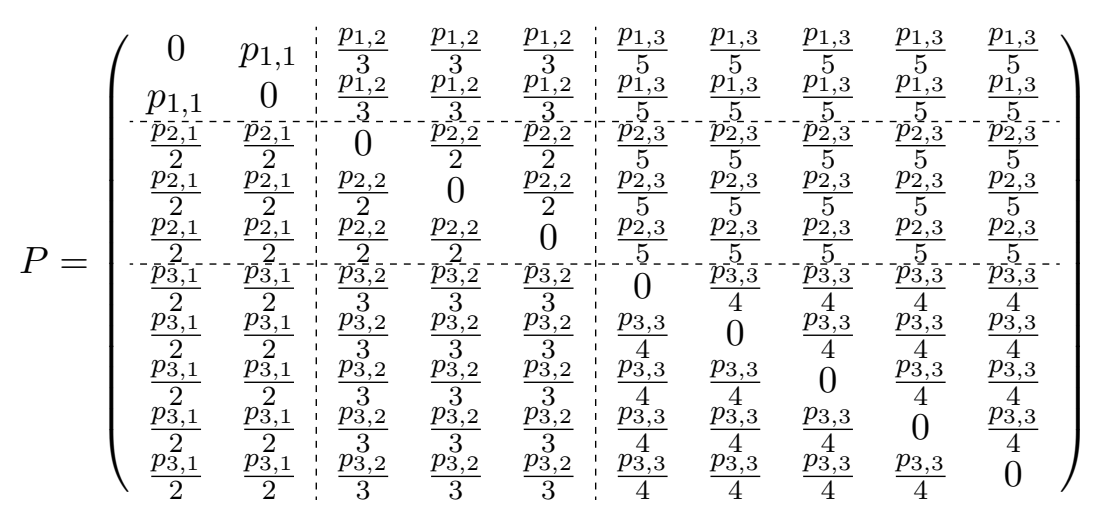

3. Main results. In this paper we obtain quantitative statements on the set of misclassified states,

$$
\mathcal{E} \triangleq \bigcup_{k=1}^{K} \hat{\mathcal{V}}_{\gamma^{\mathrm{opt}}(k)} \backslash \mathcal{V}_{k} \quad \text { where } \quad \gamma^{\mathrm{opt}} \in \arg \min _{\gamma \in \operatorname{Perm}(K)}\left|\bigcup_{k=1}^{K} \hat{\mathcal{V}}_{\gamma(k)} \backslash \mathcal{V}_{k}\right|
$$

Here, the sets $\hat{\mathcal{V}}_{1}, \ldots, \hat{\mathcal{V}}_{K}$ will always denote an approximate cluster assignment obtained from some clustering algorithm. For notational convenience we will always number the approximate clusters so as to minimize the number of misclassifications, allowing us to forego defining it formally via a permutation.

3.1. Information theoretical lower bound. Our results identify an important information quantity $I(\alpha, p) \geq 0$ that measures how difficult it is to cluster in a BMC. Its role will become clear in Theorem 1. The reason we call it an information quantity stems from fact that we have derived it as the leading coefficient in an asymptotic expansion of a log-likelihood function. Note that while it resembles one, this information quantity is not a Kullback-Leibler divergence. The individual terms are weighted according to the equilibrium distribution $\pi$, and there are two extra terms.

imsart-aos ver. 2014/10/16 file: JS_AP_SY_CBMC__arXiv.tex date: July 31, 2019 
Definition. For $\alpha \in \Delta^{K-1}$ and $p \in \Delta^{(K-1) \times K}$, let

$$
I(\alpha, p) \triangleq \min _{a \neq b} I_{a, b}(\alpha, p),
$$

where $I_{a, b}(\alpha, p) \triangleq\left\{\sum_{k=1}^{K} \frac{1}{\alpha_{a}}\left(\pi_{a} p_{a, k} \ln \frac{p_{a, k}}{p_{b, k}}+\pi_{k} p_{k, a} \ln \frac{p_{k, a} \alpha_{b}}{p_{k, b} \alpha_{a}}\right)+\left(\frac{\pi_{b}}{\alpha_{b}}-\frac{\pi_{a}}{\alpha_{a}}\right)\right\}$. Here $\pi$ denotes the solution to $\pi^{\mathrm{T}} p=\pi^{\mathrm{T}}$.

THEOREM 1. An algorithm is $(\varepsilon, c)$-locally good at $(\alpha, p)$ if it satisfies $\mathbb{E}_{P}[|\mathcal{E}|] \leq \varepsilon$ for all BMC models constructed from the given $p$ and partitions satisfying || $\mathcal{V}_{k}\left|-\alpha_{k} n\right| \leq c$ for all $k$. Assume that $T=\omega(n)$. Then there exists a strictly positive and finite constant $C$ independent of $n$ such that: there exists no $(\varepsilon, 1)$-locally good clustering algorithm at $(\alpha, p)$ when

$$
\varepsilon<C n \exp \left(-I(\alpha, p) \frac{T}{n}(1+o(1))\right) .
$$

Theorem 1 allows us to state necessary conditions for the existence of $(\varepsilon, 1)$-locally good clustering algorithms at $(\alpha, p)$ that either detect clusters asymptotically accurately, namely with $\mathbb{E}_{P}[|\mathcal{E}|]=o(n)$, or recover clusters asymptotically exactly, i.e., with $\mathbb{E}_{P}[|\mathcal{E}|]=o(1)$.

Conditions for asymptotically accurate detection. In view of the lower bound in Theorem 1 , there may exist asymptotically accurate $(\varepsilon, 1)$-locally good clustering algorithms at $(\alpha, p)$ only if $I(\alpha, p)>0$ and $T=\omega(n)$.

Conditions for asymptotically exact detection. Necessary conditions for the existence of an asymptotically exact algorithm are $I(\alpha, p)>0$ and $T-\frac{n \ln (n)}{I(\alpha, p)}=\omega(1)$. In particular, $T$ must be larger than $n \ln (n)$. We refer to the scenario where $T$ is of the order $n \ln (n)$ as the critical regime. In this regime when $T=n \ln (n)$, the necessary condition for exact recovery is $I(\alpha, p)>1$.

Note that qualitatively, the above conditions on the number of observations for accurate and exact recovery are similar to those in the SBM. In the latter, the average degrees of vertices should be such that the average total number of edges is $\omega(n)$ [10] for accurate detection, whereas this average must be at least $c n \ln (n)$ for exact recovery[13]. Here, $c$ is known and depends on the parameters of the SBM.

The information quantity $I(\alpha, p)$ for $K=2$ clusters. In the case of two clusters, we study the set of parameters $(\alpha, p)$ of the BMCs for which $I(\alpha, p)>0$ and $I(\alpha, p)>1$, the latter condition being necessary in the critical regime when $T=n \ln (n)$. 
A system with two clusters can be specified entirely with three parameters: $\alpha_{2}, p_{1,2}$, and $p_{2,1}$. Examining the explicit expression for (10) in this case, we can conclude that $I(\alpha, p)=0$ if and only if $\alpha_{2}=p_{1,2}=1-p_{2,1}$. Asymptotic accurate (resp. exact) recovery seems thus possible as soon as $T=\omega(n)$ (resp. $T=\omega(n \ln (n)))$ for almost any BMC with two clusters - the only exception are BMCs with parameters on this line. Note that if we did not have the information quantity at our disposal, it would be challenging to give a heuristic argument whether a specific BMC allows for asymptotic exact recovery. Consider for instance a BMC with $\alpha_{1}=\alpha_{2}=\frac{1}{2}$ and $p_{1,2}=1-p_{2,1} \neq \frac{1}{2}$ and $p_{1,2}>p_{2,1}$ w.l.o.g. In this scenario, $P_{x, z}=P_{y, z}$ for all $x, y, z \in \mathcal{V}$, that is, every row of the kernel is identical to any other row. Looking at this kernel, we would not expect to be able to cluster. However here $\pi_{2}>\pi_{1}$, and we could cluster based on the equilibrium distribution as $T \rightarrow \infty$. The information quantity takes the fact that we are dealing with a Markov chain appropriately into account, and correctly asserts for this case that asymptotic recovery is possible.

Figure 3 illustrates for which parameters one can possibly recover the two clusters asymptotically exactly when $T=n \ln n$. Specifically, it depicts all parameters $\alpha_{2}, p_{1,2}, p_{2,1} \in(0,1)$ for which $I(\alpha, p)>1$. If we fix $\alpha_{2}$, note that when $p_{1,2}, p_{2,1} \downarrow 0$ (bottom left), the Markov chain tends to stay within the current cluster for a substantial time. Similarly when $p_{1,2}, p_{2,1} \uparrow 1$ (top right), the Markov chain tends to jump into the other cluster every time. In both scenarios, the states are relatively easy to cluster. This draws parallels with the SBM. When either $p_{1,2} \downarrow 0$ (left) or $p_{2,1} \downarrow 0$ (bottom), clustering is again doable: in these scenarios, the Markov chain tends to stay in the cluster of the starting state - and the fact that you never see the other vertices suggests that they have other transitions rates and therefore belong to the other cluster.

3.2. Procedure for cluster recovery. Theorem 1 has established necessary conditions for asymptotically accurate and exact recovery, and has identified performance limits satisfied by any $(\varepsilon, 1)$-locally good clustering algorithm at $(\alpha, p)$. In this section, we devise a $(\varepsilon, 1)$-locally good clustering algorithm at $(\alpha, p)$ that reaches these limits order-wise. The proposed algorithm proceeds in two steps: The first step performs a spectral decomposition of the random matrix $\hat{N}$ corresponding to the empirical transition rates between any pair of states, and defined by

$$
\hat{N}_{x, y} \triangleq \sum_{t=0}^{T-1} \mathbb{1}\left[X_{t}=x, X_{t+1}=y\right] \quad \text { for } \quad x, y \in \mathcal{V}
$$

imsart-aos ver. 2014/10/16 file: JS_AP_SY__CBMC__arXiv.tex date: July 31, 2019 


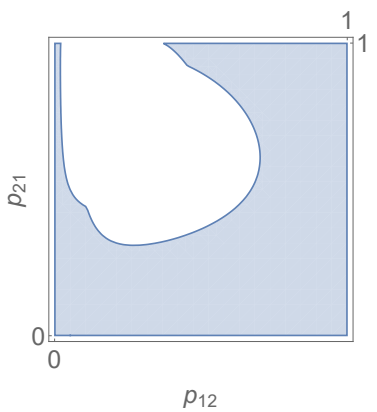

$\alpha_{2}=1 / 4$

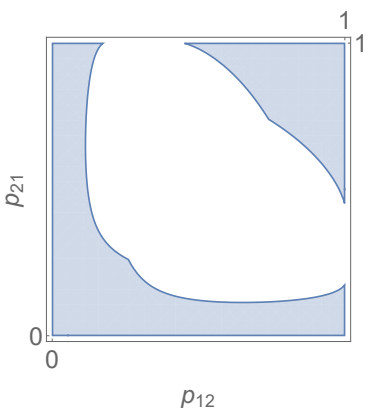

$\alpha_{2}=1 / 2$

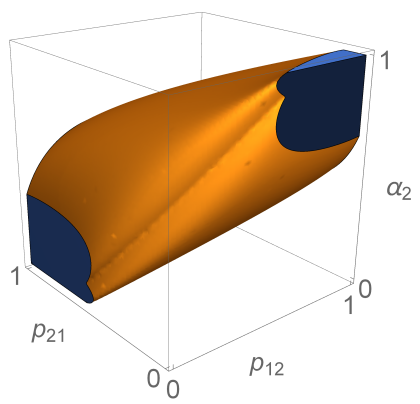

Region where $I(\alpha, p)<1$.

Fig 3: (left, middle) The parameters $\left(p_{1,2}, p_{2,1}\right)$ in blue for which asymptotic exact recovery should be possible in the critical regime $T=n \ln n$ for $K=2$ clusters. (right) The parameters $\left(\alpha_{2}, p_{1,2}, p_{2,1}\right)$ for which asymptotic exact recovery is likely not possible, i.e., $I(\alpha, p)<1$.

The rank- $K$ approximation of $\hat{N}$ is used to get initial estimates of the clusters. The second step sequentially improves the cluster estimates. In each iteration, the parameters of the BMCs are inferred from the previous cluster estimates, and states are re-assigned to clusters based on these estimated parameters and the observed trajectory (by maximizing a log-likelihood).

3.2.1. Spectral Clustering Algorithm. The first step of our procedure is the Spectral Clustering Algorithm, presented in Algorithm 1. It leverages the spectral decomposition of $\hat{N}$ to estimate the clusters.

Before applying a singular value decomposition (SVD) to $\hat{N}$, we first need to trim the matrix so as to remove states that have been visited abnormally often. These states would namely perturb the spectral decomposition of $\hat{N}$. More precisely, we define the set $\Gamma$ of states obtained from $\mathcal{V}$ by removing the $\lfloor n \exp (-(T / n) \ln (T / n))\rfloor$ states with the highest numbers of visits in the observed sample path of length $T$. The spectral decomposition is applied to the matrix $\hat{N}_{\Gamma}$ obtained from $\hat{N}$ by setting all entries on the rows and columns corresponding to states not in $\Gamma$ to zero.

The SVD of $\hat{N}_{\Gamma}$ is $U \Sigma V^{\mathrm{T}}$, from which we deduce $\hat{R}$ the best rank-K approximation of $\hat{N}_{\Gamma}: \hat{R} \triangleq \sum_{k=1}^{K} \sigma_{k} U_{\cdot, k} V_{\cdot, k}^{\mathrm{T}}$, where the values $\sigma_{1} \geq \sigma_{2} \geq$ $\cdots \geq \sigma_{n} \geq 0$ denote the singular values of $\hat{N}_{\Gamma}$ in decreasing order. We apply a clustering algorithm to the rows and columns of $\hat{R}$ to determine the clusters. While in practice you may choose to use a different algorithm, for the analysis 
we use the following: first we calculate the neighborhoods

$$
\mathcal{N}_{x} \triangleq\left\{y \in \mathcal{V} \mid \sqrt{\left\|\hat{R}_{x, \cdot}-\hat{R}_{y, \cdot}\right\|_{2}^{2}+\left\|\hat{R}_{\cdot, x}-\hat{R}_{\cdot, y}\right\|_{2}^{2}} \leq \frac{1}{n} \cdot\left(\frac{T}{n}\right)^{3 / 2}\left(\ln \frac{T}{n}\right)^{4 / 3}\right\}
$$

for $x \in \mathcal{V}$. Then we initialize $\hat{\mathcal{V}}_{k} \leftarrow \emptyset$ for $k=1, \ldots, K$ and sequentially select $K$ centers $z_{1}^{*}, \ldots, z_{K}^{*} \in \mathcal{V}$ from which we construct approximate clusters. Specifically, we iterate for $k=1, \ldots K$ :

$$
\hat{\mathcal{V}}_{k} \leftarrow \mathcal{N}_{z_{k}^{*}} \backslash\left\{\cup_{l=1}^{k-1} \hat{\mathcal{V}}_{l}\right\} \quad \text { where } \quad z_{k}^{*} \triangleq \arg \max _{x \in \mathcal{V}}\left|\mathcal{N}_{x} \backslash\left\{\cup_{l=1}^{k-1} \hat{\mathcal{V}}_{l}\right\}\right|
$$

Any remaining state is finally associated to the center closest to it, i.e., we iterate for $y \in\left\{\cup_{k=1}^{K} \hat{\mathcal{V}}_{k}\right\}^{\mathrm{c}}$

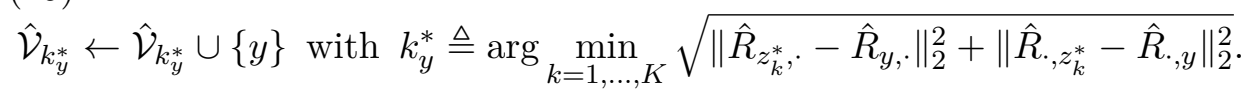

Finally, the Spectral Clustering Algorithm outputs $\hat{\mathcal{V}}_{k}$ for $k=1, \ldots, K$. Theorem 2 provides an upper bound on the number of misclassified states after executing the algorithm.

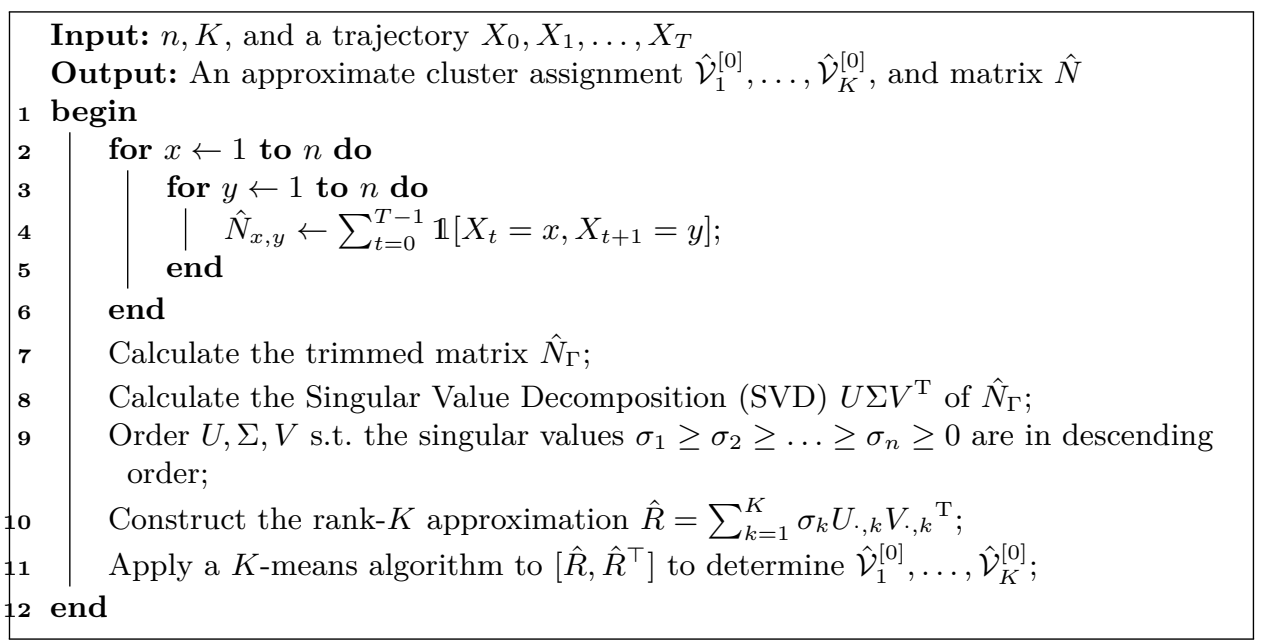

Algorithm 1: Pseudo-code for the Spectral Clustering Algorithm.

Theorem 2. Assume that $T=\omega(n)$ and $I(\alpha, p)>0$. Then the proportion of misclassified states after the Spectral Clustering Algorithm satisfies:

$$
\frac{|\mathcal{E}|}{n}=O_{\mathbb{P}}\left(\frac{n}{T} \ln \frac{T}{n}\right)=o_{\mathbb{P}}(1)
$$

imsart-aos ver. 2014/10/16 file: JS_AP_SY_CBMC__arXiv.tex date: July 31, 2019 
From the above theorem, we conclude that the first step of our recovery procedure (i.e., the Spectral Clustering Algorithm) alone achieves an asymptotically accurate detection whenever this is at all possible, say when $I(\alpha, p)>0$ and $T=\omega(n)$. However, it fails at ensuring asymptotic exact recovery, even in certain cases of $T=\omega(n \ln (n))$, and we cannot guarantee that its recovery rate approaches the fundamental limit identified in Theorem 1.

3.2.2. Cluster Improvement Algorithm. The second step of our clustering procedure, referred to as the Cluster Improvement Algorithm, aims at sequentially improving the cluster estimates obtained from the Spectral Clustering Algorithm until the recovery rate approaches the limits predicted in Theorem 1. The pseudo-code of the Cluster Improvement Algorithm is presented in Algorithm 2.

The Cluster Improvement Algorithm works as follows. Given a cluster assignment $\left\{\hat{\mathcal{V}}_{k}^{[t]}\right\}_{k=1, \ldots, K}$ obtained after the $t$-th iteration, it first calculates the estimates

$$
\begin{aligned}
& \hat{p}_{a, b}=\hat{N}_{\hat{\mathcal{V}}_{a}^{[t]}, \hat{\mathcal{V}}_{b}^{[t]}} / \hat{N}_{\hat{\mathcal{V}}_{a}^{[t]}, \mathcal{V}} \quad \text { for } \quad a, b=1, \ldots, K, \\
& \hat{\pi}_{k}=\frac{1}{T} \sum_{x \in \hat{\mathcal{V}}_{k}^{[t]}} \sum_{y \in \mathcal{V}} \hat{N}_{x, y} \quad \text { and } \quad \hat{\alpha}_{k}=\frac{\left|\hat{\mathcal{V}}_{k}^{[t]}\right|}{n} \quad \text { for } \quad k=1, \ldots, K \text {. }
\end{aligned}
$$

It then initializes $\hat{\mathcal{V}}_{k}^{[t+1]}=\emptyset$ for $k=1, \ldots, K$, and assigns each state $x=$ $1, \ldots, n$ to $\mathcal{V}_{c_{x}^{\text {opt }}}^{[t+1]} \leftarrow \mathcal{V}_{c_{x}^{\text {opt }}}^{[t+1]} \cup\{x\}$, where $c_{x}^{\text {opt }} \triangleq \arg \max _{c=1, \ldots, K} u_{x}^{[t]}(c)$, and

$$
u_{x}^{[t]}(c) \triangleq\left\{\sum_{k=1}^{K}\left(\hat{N}_{x, \hat{\mathcal{V}}_{k}^{[t]}} \ln \hat{p}_{c, k}+\hat{N}_{\hat{\mathcal{V}}_{k}^{[t]}, x} \ln \frac{\hat{p}_{k, c}}{\hat{\alpha}_{c}}\right)-\frac{T}{n} \cdot \frac{\hat{\pi}_{c}}{\hat{\alpha}_{c}}\right\} .
$$

This results in a new cluster assignment $\left\{\hat{\mathcal{V}}_{k}^{[t+1]}\right\}_{k=1, \ldots, K}$. Note that the algorithm works by placing each state in the cluster it most likely belongs to, based on the known structure and the sample path. This can be seen by noting that the objective function in (18) is the difference between two log-likelihood functions.

The second step of our clustering procedure applies the Cluster Improvement Algorithm several times, using as the initial input the cluster assignment $\left\{\hat{\mathcal{V}}_{k}^{[0]}\right\}_{k=1, \ldots, K}$ obtained from the Spectral Clustering Algorithm. We denote by $\mathcal{E}^{[t]}$ the set of misclassified state after the $t$-th iteration of the Clustering Improvement Algorithm. The overall performance of the clustering procedure is quantified in Theorem 3.

imsart-aos ver. 2014/10/16 file: JS_AP_SY_CBMC__arXiv.tex date: July 31, 2019 


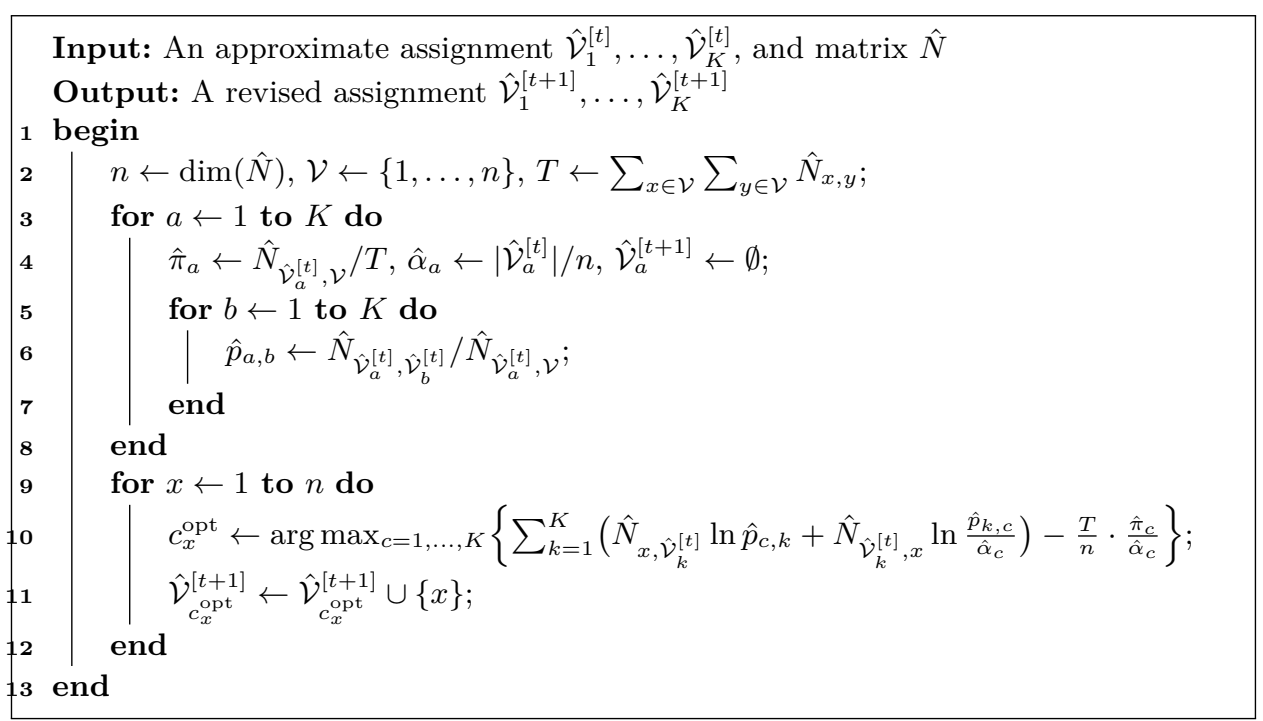

Algorithm 2: Pseudo-code for the Cluster Improvement Algorithm.

Theorem 3. Assume that $T=\omega(n)$ and $I(\alpha, p)>0$. Then for any $t \geq 1$, after $t$ iterations of the Clustering Improvement Algorithm, initially applied to the output of the Spectral Clustering Algorithm, we have:

$$
\frac{\left|\mathcal{E}^{[t]}\right|}{n}=O_{\mathbb{P}}\left(\mathrm{e}^{-t\left(\ln \frac{T}{n}-\ln \ln \frac{T}{n}\right)}+\mathrm{e}^{-\frac{\alpha_{\min }^{2}}{720 \eta^{3} \alpha_{\max }^{2}} \frac{T}{n} I(\alpha, p)}\right) .
$$

Observe that for $t=\ln n$, the number of misclassified states after applying $t$ times the Clustering Improvement Algorithm is at most of the order $n \exp (-C(T / n) I(\alpha, p))$ with $C \triangleq \frac{\alpha_{\min }^{2}}{720 \eta^{3} \alpha_{\max }^{2}}$. Up to the constant $C$, this corresponds to the fundamental recovery rate limit identified in Theorem 1 . In particular, our clustering procedure achieves asymptotically exact detection under the following nearly tight sufficient condition: $I(\alpha, p)>0$ and $T-$ $\frac{n \ln n}{C \cdot I(\alpha, p)}=\omega(1)$.

4. Numerical experiments. In this section, we numerically assess the performance of our algorithms. We first investigate a simple illustrative example. Then we study the sensitivity of the error rate of the Spectral Clustering Algorithm w.r.t. the number of states and the length of the observed trajectory. Finally we show the performance of the Cluster Improvement Algorithm depending on the number of times it is applied to the output of the Spectral Clustering Algorithm. 
4.1. An example. Consider $n=300$ states grouped into three clusters of respective relative sizes $\alpha=(0.15,0.35,0.5)$, i.e., the cluster sizes are cluster sizes $\left|\mathcal{V}_{1}\right|=48,\left|\mathcal{V}_{2}\right|=93$ and $\left|\mathcal{V}_{3}\right|=159$. The transition rates between these clusters are defined by: $p=(0.9200,0.0450,0.0350 ; 0.0125,0.8975,0.0900$; $0.0175,0.0200,0.9625)$.

We generate a sample path of the Markov chain of length $T=n^{1.025} \ln n \approx$ 1973 and calculate $\hat{N}$. A density plot of a typical sample of $\hat{N}$ is shown in Figure 4a. The same density plot is presented in Figure $4 \mathrm{~b}$ where the states have been sorted so as states in the same cluster are neighbors. It is important to note that the algorithms are of course not aware of the structure initially sorting states constitutes their objective. Next in Figure 4c, we show a color representation of the kernel $P$ with sorted rows and columns, in which we can clearly see the groups. Note that the specific colors have no meaning, except for the fact that within the same image two entries with the same color have the same numerical value.

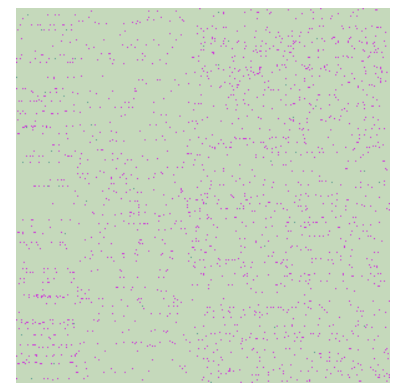

(a) $\hat{N}$, unsorted

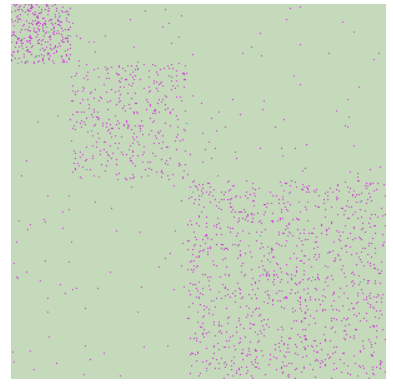

(b) $\hat{N}$, sorted

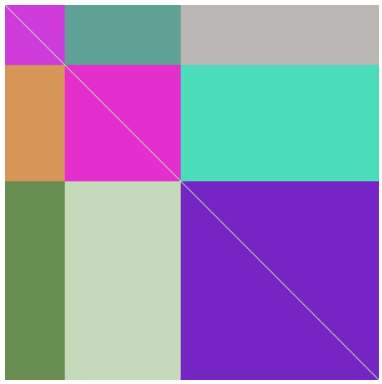

(c) $P$, sorted

Fig 4: A sample path of length $T=n^{1.025} \ln n \approx 1973$ was generated, from which $\hat{N}$ is calculated. If we sort the states according to the clusters they belong to, we can see that states within the same cluster share similar dynamics.

Next we apply the Spectral Clustering Algorithm. This generates an initial approximate clustering $\hat{\mathcal{V}}_{1}^{[0]}, \hat{\mathcal{V}}_{2}^{[0]}, \hat{\mathcal{V}}_{3}^{[0]}$ of the states. We generate a visual representation of this clustering by constructing $\hat{P}^{[0]}$ from the approximate cluster structure and the estimate $\hat{p}^{[0]}$. This represents the belief that the algorithm has at this point of the true BMC kernel $P$. A color representation of this kernel is shown in Figure 5a. We finally execute the Cluster Improvement Algorithm. After 3 iterations, it has settled on a final clustering. We generate a color representation of the clustering similar to before, resulting in Figure 5b. The algorithms achieved a 99.7\% accuracy: all but one state have been 
accurately clustered.

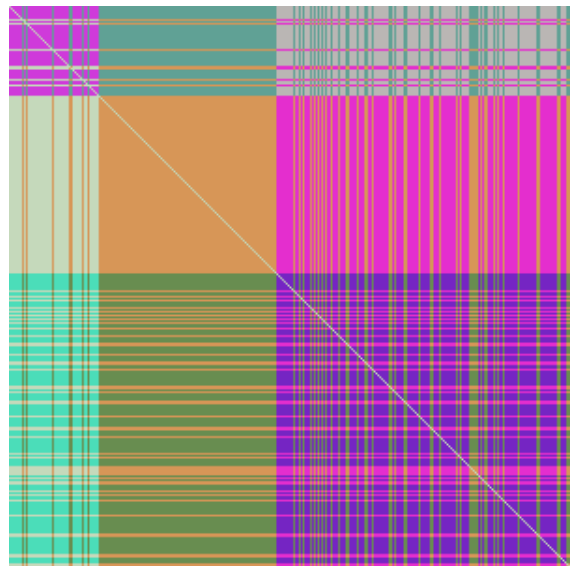

(a) Initial clustering.

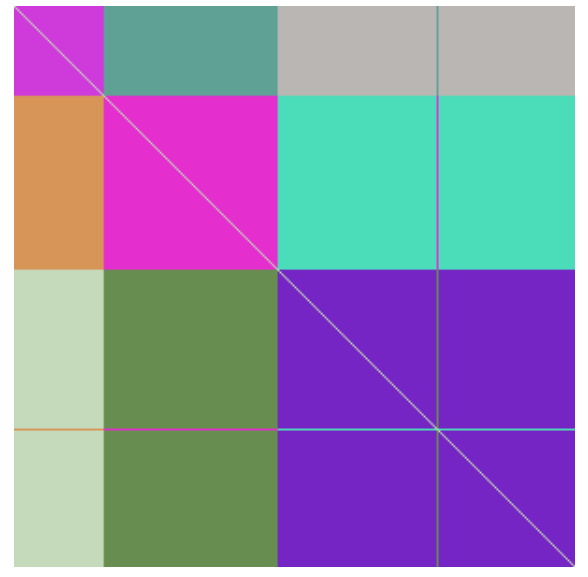

(b) Final clustering.

Fig 5: (a) Result after applying the Spectral Clustering Algorithm to the approximation $\hat{N}$. (b) Result after applying 3 iterations of the Cluster Improvement Algorithm. 99.7\% of all states were accurately clustered.

4.2. Performance sensitivity of the Spectral Clustering Algorithm. In this section, we examine the dependency of the number of misclassified states on the size of the kernel $n$, when we only apply the Spectral Clustering Algorithm. We choose $\alpha=(0.15,0.35,0.5)$, and set $p=(0.50,0.20,0.30$; $0.10,0.70,0.20 ; 0.35,0.05,0.60)$. These parameters imply that $I(\alpha, p) \approx 0.88$ $>0$. This value for $I(\alpha, p)$ is lower than in the case examined in Section 4.1, so we expect clustering to be more difficult. We have selected a more challenging model so that the initial number of misclassified states will be large and the asymptotics clear.

Figure 6 displays the error rate of the Spectral Clustering algorithm as a function of $n$, for different trajectory lengths $T$. As benchmarks, we include a dashed line that indicates the error rate obtained by assigning states to clusters uniformly at random, i.e., $\mathbb{P}\left[v \notin \mathcal{V}_{\sigma(v)}\right]=\sum_{k=1}^{K} \mathbb{P}\left[v \notin \mathcal{V}_{k} \mid \sigma(v)=\right.$ $k] \alpha_{k}=1-1 / K$, as well as a dotted line that indicates the error rate when assigning all states to the smallest cluster, i.e., $1-\min _{k}\left\{\alpha_{k}\right\}$. For the $K$ means step of the algorithms, we use Mathematica's default implementation for convenience. Observe that when $T=n \ln n$, the fraction of misclassified states hardly decrease as a function of $n$. This is in line with our lower bound. When $T$ gets larger, the error converges to zero faster. Note that the Spectral 
Clustering Algorithm recovers the clusters exactly when the sample path is sufficiently long.

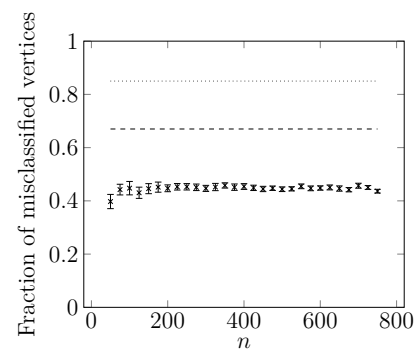

(a) $T=n \ln n$

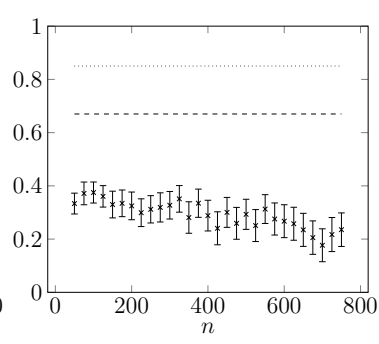

(b) $T=n(\ln n)^{3 / 2}$

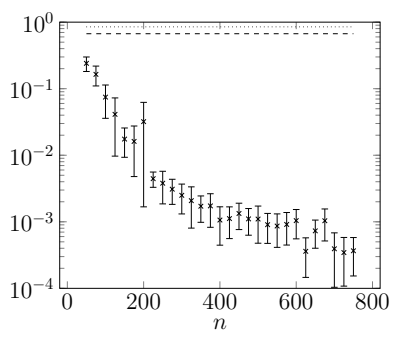

(c) $T=n(\ln n)^{2}$

Fig 6: The error rate of the Spectral Clustering Algorithm as function of $n$, for different scalings of $T$. Every point is the average result of 40 simulations, and the bars indicate a 95\%-confidence interval.

4.3. Performance sensitivity of the Cluster Improvement algorithm. We now examine the number of misclassified states as a function of $T$, when we apply the Spectral Clustering Algorithm and a certain number of iterations of the Cluster Improvement Algorithm. We choose $\alpha=(1 / 3,1 / 3,1 / 3)$, and set $p=(0.1,0.4,0.5 ; 0.7,0.1,0.2 ; 0.6,0.3,0.1)$. Different from the previous experiments, the clusters are now of equal size and the off-diagonal entries of $p$ are dominant. These parameters imply that $I(\alpha, p) \approx 0.27>0$, so the cluster algorithms should work, but the situation is again more challenging than in Section 4.1 and Section 4.2.

Figure 7 depicts the error after applying the Spectral Clustering Algorithm and subsequently the Cluster Improvement Algorithm up to two times, as a function of $T$. We have chosen both $n, T$ relatively small so that the inputs are significantly noisy. For short sample paths, $T \lesssim 15000$, the data is so noisy that the Cluster Improvement Algorithm does not provide any improvement over the Spectral Clustering Algorithm. For $T \gtrsim 15000$, the Spectral Clustering Algorithm provides a sufficiently accurate initial clustering for the Cluster Improvement Algorithm to work. Because marks 1 and 2 overlap in almost all cases, we can conclude that there is (on average, and in the present situation) no benefit in running the Clustering Improvement Algorithm more than once. There is no mark 2 at $T=30000$ in this logarithmic plot, because the Cluster Improvement Algorithm achieved $100 \%$ accurate detection after 2 iterations in all 200 simulations. 
4.4. Critical regime where $T=n \ln (n)$. We now study how well our clustering procedure performs in the critical regime $T=n \ln n$. Here, we will consider $K=2$ clusters of equal size: $\alpha_{1}=\alpha_{2}=\frac{1}{2}$. Recall that every such BMC can then be completely parameterized by $\left(p_{1,2}, p_{2,1}\right) \in(0,1)^{2}$. Our goal in this section is to numerically evaluate $\hat{\mathcal{F}}_{1}(\varepsilon)=\left\{\left(p_{1,2}, p_{2,1}\right) \in\right.$ $\left.(0,1)^{2} \mid \mathbb{E}_{P}\left[\left|\mathcal{E}^{[t]}\right| / n\right] \geq \varepsilon\right\}$ as a proxy estimate to the region $\left\{\left(p_{1,2}, p_{2,1}\right) \mid I(\alpha, p)\right.$ $\leq 1\}$. We rasterized $(0,1)^{2}$ and ran our clustering procedure for $n=300$ with $t=6$ improvement steps for each parameter pair $\left(p_{1,2}, p_{2,1}\right)$. The results are shown in Figure 8. Note that the sample mean at each rasterpoint was calculated from 10 independent runs.

5. Lower bounds via change-of-measure arguments. In this section, we prove Theorem 1 using change-of-measure arguments such as those frequently used to identify information-theoretical limits in online stochastic optimization [19]. We sketch the proof below, and provide a detailed description of its steps later in this section. We distinguish two cases:

(i) If $I(\alpha, p)=0$, we prove that there are two clusters whose states cannot be distinguished from any set of observations. Hence, the number of misclassified states grows linearly with $n$, which corresponds to the statement of the theorem when $I(\alpha, p)=0$.

(ii) If $I(\alpha, p)>0$, we use a change-of-measure argument. We denote by $\Phi$ the true stochastic model under which the observations $X_{0}, \ldots, X_{T}$ are generated, and by $\mathbb{P}_{\Phi}$ (resp. $\mathbb{E}_{\Phi}$ ) the corresponding probability measure (resp. expectation). The true model is characterized by the transition matrix $P$. In a change-of-measure argument, we pretend that the observations are instead generated under a slightly different stochastic model $\Psi$ (denote by $\mathbb{P}_{\Psi}$ and $\mathbb{E}_{\Psi}$ the corresponding probability measure and expectation). The modified stochastic model $\Psi$ is constructed as follows. We pick a state $V^{*}$ randomly from clusters $\mathcal{V}_{a}$ and $\mathcal{V}_{b}$ with $a \neq b$, and place this state in its own cluster, indexed by 0 . We further construct a transition matrix $Q$ depending on $V^{*}$ and slightly different than $P . \Psi$ is hence characterized by both $V^{*}$ and the transition matrix $Q$. Next, we introduce the log-likelihood ratio of the observations $X_{0}, \ldots, X_{T}$ under the transition matrices $P$ and $Q$ :

$$
L \triangleq \ln \frac{\mathbb{P}_{Q}\left[X_{0}, X_{1}, \ldots, X_{T}\right]}{\mathbb{P}_{P}\left[X_{0}, X_{1}, \ldots, X_{T}\right]},
$$

where

$\mathbb{P}_{P}\left[X_{0}, X_{1}, \ldots, X_{T}\right]=\prod_{t=1}^{T} P_{X_{t-1}, X_{t}}$, and $\mathbb{P}_{Q}\left[X_{0}, X_{1}, \ldots, X_{T}\right]=\prod_{t=1}^{T} Q_{X_{t-1}, X_{t}}$.

imsart-aos ver. 2014/10/16 file: JS_AP_SY__CBMC__arXiv.tex date: July 31, 2019 


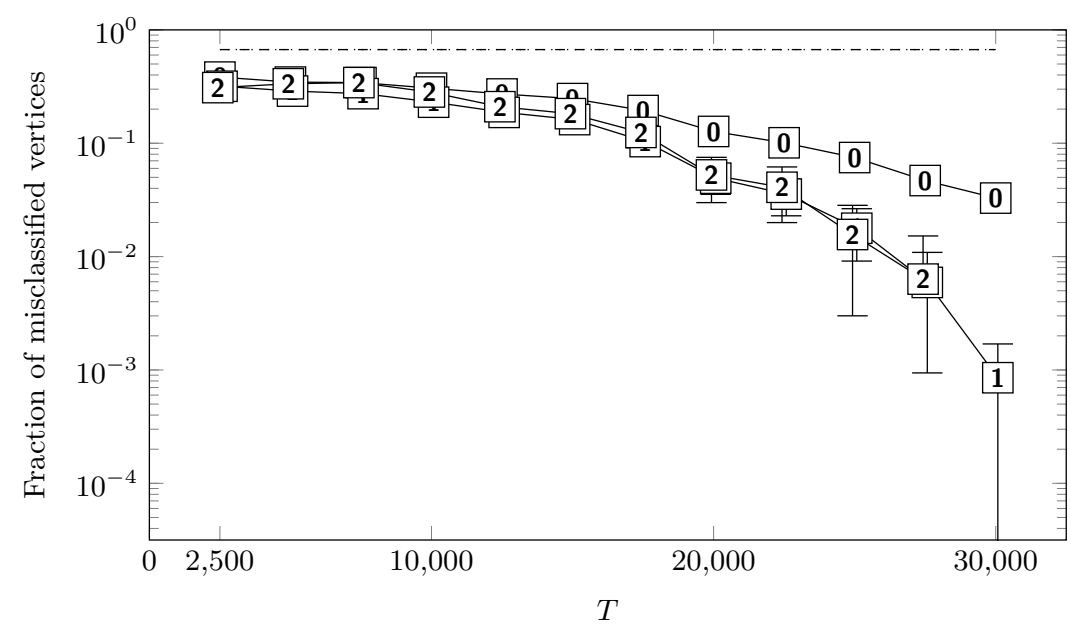

Fig 7: The error after applying the Spectral Clustering Algorithm (mark 0), and subsequently the Cluster Improvement Algorithm (marks 1,2) several times, as a function of $T$. Each number represents the number of improvement steps. Here, $n=240$. Every point is the average result of 200 simulations, and the bars indicate a 95\%-confidence interval. We have minorly offset marks 1,2 to the right and left for readability, respectively. At $T=30000$, the Cluster Improvement Algorithm achieved 100\% accurate detection after 2 iterations in all 200 instances.

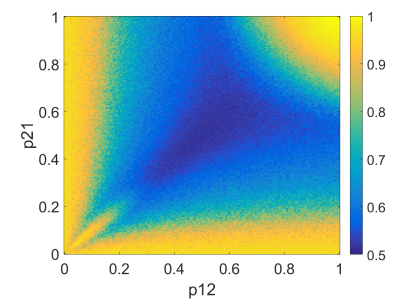

After the SCA.

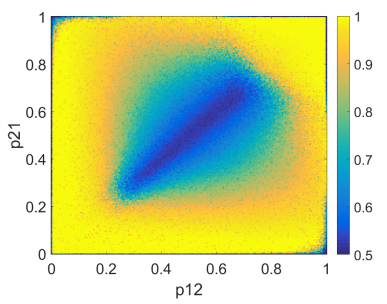

After the CIA.

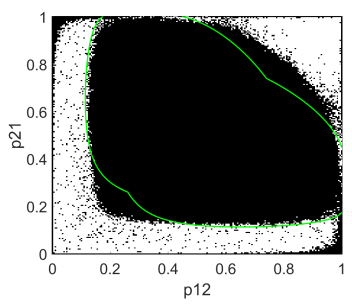

$\hat{\mathcal{F}}_{1}(\varepsilon=0.027)$

Fig 8: The average proportion of well-classified states for each rasterpoint $\left(p_{1,2}, p_{2,1}\right) \in(0,1)^{2}$ after the Spectral Clustering Algorithm (left) and Cluster Improvement Algorithm (middle), and numerical feasibility region of our clustering procedure (right), both in the critical regime $T=n \ln n$. The line outlines the theoretical region $I(\alpha, p) \leq 1$ within which no algorithm exists able to asymptotically recover the clusters exactly. 
Note that $L$ is random because it depends on the observations, but also on $V^{*}$. We then establish that there exist constants $\delta, C>0$ (independent of $n$ ) such that under any clustering algorithm the expected number of misclassified states satisfies:

$$
\mathbb{E}_{\Phi}[|\mathcal{E}|] \geq C n \exp \left(-\mathbb{E}_{\Psi}[L]-\sqrt{\frac{2}{\delta}} \sqrt{\operatorname{Var}_{\Psi}[L]}\right) .
$$

Finally for particular choices of $a, b$ and the transition matrix $Q$, the analysis of $\mathbb{E}_{\Psi}[L]$ and $\operatorname{Var}_{\Psi}[L]$ reveals that: for $T=\omega(n), \mathbb{E}_{\Psi}[L] \leq(T / n) I(\alpha, p)+$ $o(T / n)$ and $\operatorname{Var}_{\Psi}[L]=o\left(T^{2} / n^{2}\right)$. Combining this with (30) completes the proof of Theorem 1.

\subsection{Necessary and sufficient condition for $I(\alpha, p)=0$.}

Lemma 1. For any $B M C, I(\alpha, p)=0$ if and only if there exists $i \neq j$ such that $p_{i, c}=p_{j, c}$ and $p_{c, i} / \alpha_{i}=p_{c, j} / \alpha_{j}$ for all $c \in\{1, \ldots, K\}$.

Lemma 1 implies that observing a trajectory of the Markov chain does not provide any information allowing us to distinguish between states in $\mathcal{V}_{i}$ and $\mathcal{V}_{j}$. As a consequence, any clustering algorithm will misclassify at least a fixed proportion of states. The proof of Lemma 1 is provided in §SM3.

5.2. Change-of-measure argument. In the remainder of this section, we assume that $I(\alpha, p)>0$. The argument consists in considering that the observations $X_{0}, \ldots, X_{T}$ are generated by a slightly different stochastic model than the true model defined by the clusters and the transition matrix $P$. As mentioned above, the modified model is obtained by randomly choosing a state $V^{*}$ from two clusters $\mathcal{V}_{a}$ and $\mathcal{V}_{b}$, and by constructing a transition matrix $Q$ depending on $V^{*}$ that is slightly different from $P$. Under the new model, $V^{*}$ is placed in its own cluster indexed by 0 . The matrix $Q$ is parametrized by $q$, a $2(K+1)$-dimensional vector in

$$
\mathcal{Q} \triangleq\left\{\left(q_{k, 0}, q_{0, k}\right)_{k=0, \ldots, K} \in(0, \infty) \mid q_{0,0}=0, \sum_{l=1}^{K} q_{0, l}=1\right\}
$$

where $q_{k, 0}$ (resp. $\left.q_{0, k}\right)$ will define the probability to move from a state in cluster $\mathcal{V}_{k}$ to $V^{*}$ (resp. from state $V^{*}$ to a state in $\mathcal{V}_{k}$ ) under the new model $\Psi$. We also define:

$$
q_{k, l} \triangleq p_{k, l}-\frac{q_{k, 0}}{K n} \quad \text { for } \quad k, l=1, \ldots, K
$$

imsart-aos ver. 2014/10/16 file: JS_AP_SY__CBMC_-arXiv.tex date: July 31, 2019 
and assume that $n>\left\lceil\max _{k, l=1, \ldots, K}\left\{q_{k, 0} /\left(K p_{k, l}\right)\right\}\right\rceil$ so that the entries of (24) are strictly positive. Note that the collection $\left\{q_{k, l}\right\}_{k, l \in\{0,1, \ldots, K\}}$ does not constitute a stochastic matrix, but does resemble the transition matrix $p$ for sufficiently large $n$. We are now ready to define $Q$ component-wise:

$$
Q_{x, y} \triangleq \frac{q_{\omega(x), \omega(y)} \mathbb{1}[x \neq y]}{\left|\mathcal{W}_{\omega(y)}\right|-\mathbb{1}[\omega(x)=\omega(y)]}, \quad Q_{x, V^{*}} \triangleq \frac{q_{\omega(x), 0}}{n} \quad \text { for } \quad x \in \mathcal{V}, y \neq V^{*},
$$

where

$$
\omega(x) \triangleq\left\{\begin{array} { l l } 
{ 0 } & { \text { if } x = V ^ { * } , } \\
{ \sigma ( x ) } & { \text { if } x \neq V ^ { * } }
\end{array} , \quad \text { and } \quad \mathcal { W } _ { k } \triangleq \left\{\begin{array}{ll}
\left\{V^{*}\right\} & \text { if } k=0, \\
\mathcal{V}_{k} \backslash\left\{V^{*}\right\} & \text { if } k=1, \ldots, K,
\end{array}\right.\right.
$$

for notational convenience. This has the added benefit of giving (25) a similar form as (4).

$Q$ is by construction a stochastic matrix (see §SM3.1). Note furthermore that because $Q$ is constructed from $P$, which by assumption describes an irreducible Markov chain, and because the entries $\left\{q_{k, 0}, q_{0, k}\right\}_{k=1, \ldots, K}$ are all strictly positive, $Q$ also describes an irreducible Markov chain. Next, we highlight other basic properties of this chain, and provide an example of matrix $Q$.

Equilibrium distribution. Let $\Pi^{(Q)}$ denote the equilibrium distribution of a Markov chain with transition matrix $Q$, i.e., the solution to $\Pi^{(Q) \mathrm{T}} Q=\Pi^{(Q) \mathrm{T}}$. By symmetry of states in the same cluster $\Pi_{x}^{(Q)}=\Pi_{y}^{(Q)} \triangleq \bar{\Pi}_{k}^{(Q)}$ for any two states $x, y \in \mathcal{W}_{k}$ and all $k \in\{0,1, \ldots, K\}$. Define

$$
\gamma_{k}^{[0]} \triangleq \lim _{n \rightarrow \infty} \sum_{x \in \mathcal{W}_{k}} \Pi_{x}^{(Q)}=\lim _{n \rightarrow \infty}\left|\mathcal{W}_{k}\right| \bar{\Pi}_{k}^{(Q)} \quad \text { for } \quad k \in\{0,1, \ldots, K\} .
$$

We can expect $\gamma_{0}^{[0]}$ to be zero, because by our construction of $Q$ we can expect that $\Pi_{x}^{(Q)}=O(1 / n)$ for all $x \in \mathcal{V}$ (including $V^{*}$ ). We therefore also define its higher order statistic $\gamma_{0}^{[1]} \triangleq \lim _{n \rightarrow \infty} n \Pi_{V^{*}}^{(Q)}$. The following proposition relates these scaled quantities to the parameters of our BMC $\left\{X_{t}\right\}_{t \geq 0}$. The proof is deferred to §SM3.2, and relies on several applications of the balance equations and a subsequent asymptotic analysis.

Proposition 3. For $k=1, \ldots, K, \gamma_{k}^{[0]}=\pi_{k}$. Furthermore $\gamma_{0}^{[0]}=0$ and $\gamma_{0}^{[1]}=\sum_{k=1}^{K} \pi_{k} q_{k, 0}$.

Mixing time. It is important to note that Proposition 2 holds for a Markov chain with $Q$ as its transition matrix as well. This follows when applying the exact same proof.

imsart-aos ver. 2014/10/16 file: JS_AP_SY_CBMC__arXiv.tex date: July 31, 2019 
Example. It is illustrative to explicitly write down at least one example kernel $Q$. For $K=3, \alpha=(2 / 10,3 / 10,5 / 10)$ and $n=10, V^{*}=7$, it is given by

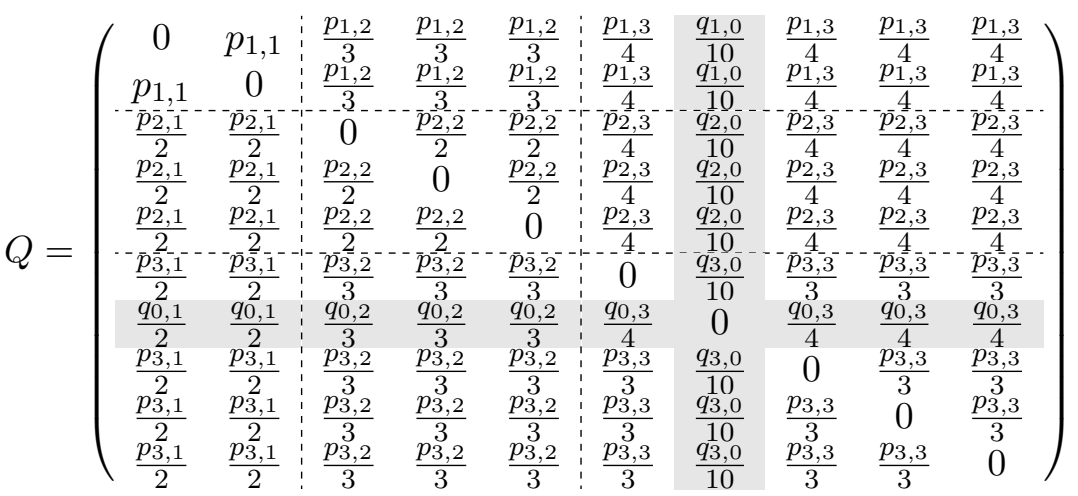

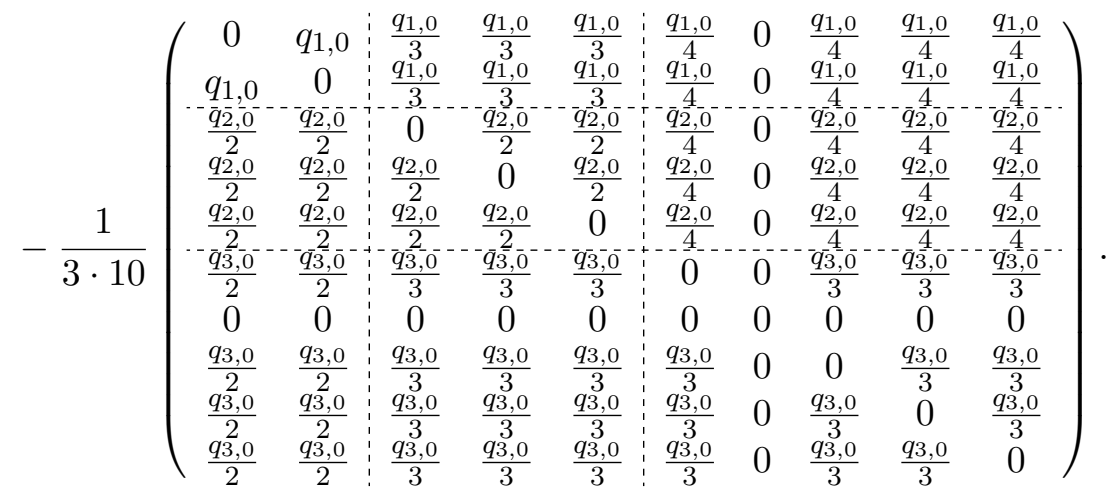

Here, we have indicated the original cluster structure in dashed lines, and we have colored the row and column corresponding to the modified cluster behavior of state $V^{*}$. Comparing (28) to (8) helps understanding how $Q$ is constructed and how $Q$ compares to $P$. Note in particular the minor changes to the normalizations of all entries.

5.3. Log-likelihood ratio and its connection to the error rate. To describe how the log-likelihood ratio $L$ relates to the error rate, we first introduce the following notations. For any $q \in \mathcal{Q}$, and any cluster index $a$, we define:

$I_{a}(q \| p) \triangleq \sum_{k=1}^{K}\left(\left(\sum_{l=1}^{K} \pi_{l} q_{l, 0}\right) q_{0, k} \ln \frac{q_{0, k}}{p_{a, k}}+\pi_{k} q_{k, 0} \ln \frac{q_{k, 0} \alpha_{a}}{p_{k, a}}\right)+\left(\frac{\pi_{a}}{\alpha_{a}}-\sum_{k=1}^{K} \pi_{k} q_{k, 0}\right)$.

As shown later in this section, $I_{a}(q \| p)$ is the leading order of the expected $\log$-likelihood ratio $L$ under $\Psi$ and given that $V^{*}$ is extracted from $\mathcal{V}_{a}$. We further define for any pair of cluster indexes $a, b: \mathcal{Q}(a, b) \triangleq\left\{q \in \mathcal{Q} \mid I_{a}(q \| p)=\right.$ $\left.I_{b}(q \| p)\right\}$. These sets are not empty as stated below.

imsart-aos ver. 2014/10/16 file: JS_AP_SY_CBMC__arXiv.tex date: July 31, 2019 
Lemma 2. For any two cluster indexes $a \neq b, \mathcal{Q}(a, b) \neq \emptyset$.

Next, Proposition 4 states that choosing $q \in \mathcal{Q}(a, b)$, one may obtain lower bounds on the error rate by analyzing the log-likelihood ratio $L$.

Proposition 4. Assume that $V^{*}$ is chosen uniformly at random from two different clusters $\mathcal{V}_{a}$ and $\mathcal{V}_{b}$, that $Q$ is constructed from $q \in \mathcal{Q}(a, b)$, and that there exists a $(\varepsilon, 1)$-locally good clustering algorithm at $(\alpha, p)$. Then:

(i) There exists a constant $\delta>0$ independent of $n$ s.t. $\mathbb{P}_{\Psi}\left[V^{*} \in \mathcal{E}\right] \geq \delta>0$.

(ii) There exists a constant $C>0$ independent of $n$ such that

$$
\mathbb{E}_{\Phi}[|\mathcal{E}|] \geq C n \exp \left(-\mathbb{E}_{\Psi}[L]-\sqrt{\frac{2}{\delta}} \sqrt{\operatorname{Var}_{\Psi}[L]}\right) .
$$

5.4. Analysis of the log-likelihood ratio. Proposition 5 characterizes the leading term of $\mathbb{E}_{\Psi}[L]$ when the cluster from which $V^{*}$ originates is fixed.

Proposition 5. For any given cluster index a, and $q \in \mathcal{Q}, \mathbb{E}_{\Psi}\left[L \mid \sigma\left(V^{*}\right)=\right.$ $a]=(T / n) I_{a}(q \| p)+o(T / n)$.

With Proposition 6, we establish that $\sqrt{\operatorname{Var}_{\Psi}\left[L \mid \sigma\left(V^{*}\right)=a\right]}$ is asymptotically negligible when compared to $\mathbb{E}_{\Psi}\left[L \mid \sigma\left(V^{*}\right)\right]$ whenever $T=\omega(n)$. The proof relies on relating the covariances between the $T$ transitions in the sample path $\left\{X_{0}, X_{1}, X_{2}, \ldots, X_{T}\right\}$ to the mixing time of the underlying Markov chain. Proposition 2 then allows us to bound variance.

Proposition 6. For any given cluster index $a$, and $q \in \mathcal{Q}$, if $T=\omega(1)$, then $\operatorname{Var}_{\Psi}\left[L \mid \sigma\left(V^{*}\right)=a\right]=O(T / n)$. As a consequence if $T=\omega(n)$, then $\operatorname{Var}_{\Psi}\left[L \mid \sigma\left(V^{*}\right)=a\right]=o\left(T^{2} / n^{2}\right)$.

Combining Propositions 4, 5, and 6, we obtain Corollary 1.

Corollary 1. There exists a constant $C>0$ such that for any two different cluster indexes $a$ and $b$, and for any $q \in \mathcal{Q}(a, b)$, if $T=\omega(n)$, then $\mathbb{E}_{\Psi}[|\mathcal{E}|] \geq C n \exp \left(-(T / n) I_{a}(q \| p)+o(T / n)\right)$.

By varying $a, b$, and $q \in \mathcal{Q}(a, b)$ in Corollary 1 , we conclude that if $T=\omega(n): \mathbb{E}_{\Phi}[|\mathcal{E}|] \geq C n \exp (-(T / n) J(\alpha, p)+o(T / n))$, where $J(\alpha, p) \triangleq$ $\min _{a \neq b} \min _{q \in \mathcal{Q}(a, b)} I_{a}(q \| p)$.

5.5. Connecting $I(\alpha, p)$ and $J(\alpha, p)$. Finally, to complete the proof of Theorem 1 , we relate $I(\alpha, p)$ to $J(\alpha, p)$ by proving that:

Lemma 3. For any $B M C$, we have $J(\alpha, p) \leq I(\alpha, p)$.

imsart-aos ver. 2014/10/16 file: JS_AP_SY_CBMC__arXiv.tex date: July 31, 2019 
6. Performance of the Spectral Clustering Algorithm. This section is devoted to the proof of Theorem 2. The Spectral Clustering Algorithm relies on a spectral decomposition of the trimmed matrix $\hat{N}_{\Gamma}$, gathering the empirical transition rates between states. The proof of Theorem 2 hence leverages concentration inequalities for Markov chains, provided in Appendix $\S \mathrm{SM} 1$, and a spectral analysis of $\hat{N}$ or $\hat{N}_{\Gamma}$.

6.1. Spectral analysis. The main ingredient in the proof of Theorem 2 is a concentration bound on the spectral norm of the matrix $\hat{N}_{\Gamma}$ centered around its mean, or more precisely on $\left\|\hat{N}_{\Gamma}-N\right\|$. The tighter such a bound is, the tighter our performance analysis of the Spectral Clustering Algorithm will be. Note that the concentration of the spectral norm holds for the trimmed matrix, i.e., a matrix based on $\hat{N}$ in which the rows and columns that correspond to states that are visited too often are set to 0.

Proposition 7. For any $B M C,\left\|\hat{N}_{\Gamma}-N\right\|=O_{\mathbb{P}}\left(\sqrt{\frac{T}{n} \ln \frac{T}{n}}\right)$.

The above concentration result, proved in §SM4.1, is sufficiently tight for the purposes of this paper, but can be improved up to logarithmic terms. The primary challenge one encounters in establishing this bound is that $\hat{N}$ is a random matrix with stochastically dependent entries, as explained in the introduction. The concentration of the entire spectrum of $\hat{N}_{\Gamma}$ would be an intriguing topic for future study.

6.2. Proof of Theorem 2. Throughout the proof, we use the following notation: $N_{x, y} \triangleq \mathbb{E}_{\Phi}\left[\hat{N}_{x, y}\right]=T \Pi_{x} P_{x, y}$ for $x, y \in \mathcal{V}, \hat{R}^{0}=\left[\hat{R}, \hat{R}^{\top}\right], N^{0}=$ $\left[N, N^{\top}\right]$, and $\hat{N}^{0}=\left[\hat{N}_{\Gamma}, \hat{N}_{\Gamma}^{\top}\right]$. We further introduce the quantity

$$
D(\alpha, p) \triangleq \min _{a, b: a \neq b} \sum_{k=1}^{K}\left(\left(\frac{\pi_{a} p_{a, k}}{\alpha_{k} \alpha_{a}}-\frac{\pi_{b} p_{b, k}}{\alpha_{k} \alpha_{b}}\right)^{2}+\left(\frac{\pi_{k} p_{k, a}}{\alpha_{k} \alpha_{a}}-\frac{\pi_{k} p_{k, b}}{\alpha_{k} \alpha_{b}}\right)^{2}\right) .
$$

Note that $D(\alpha, p)=0$ if and only if there exist $a, b$ such that $(\mathrm{C} 1) p_{a, k}=p_{b, k}$ for all $k,(\mathrm{C} 2) p_{k, a} / \alpha_{a}=p_{k, b} / \alpha_{b}$ for all $k$, and (C3) $\pi_{a} / \alpha_{a}=\pi_{b} / \alpha_{b}$. Under (C1)-(C3), $I(\alpha, p)=0$. Thus, $D(\alpha, p)>0$ when $I(\alpha, p)>0$.

The proof of Theorem 2 consists of four steps.

Step 1. We show that $N^{0}$ satisfies a separability property: i.e., if two states $x, y \in \mathcal{V}$ do not belong to the same cluster, the $l_{2}$-distance between

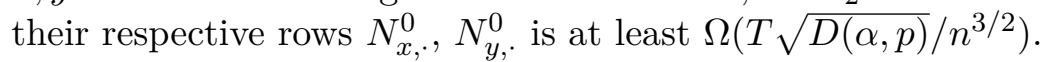

Step 2. We upper bound the error $\left\|\hat{R}^{0}-N^{0}\right\|_{\text {F }}$ using $\left\|\hat{N}_{\Gamma}-N\right\|$.

imsart-aos ver. 2014/10/16 file: JS_AP_SY_CBMC__arXiv.tex date: July 31, 2019 
Step 3. We prove that if $\left\|\hat{N}_{\Gamma}-N\right\|$ is small enough as $n$ grows large, then for any state $x$ misclassified under the Spectral Clustering Algorithm, $\left\|\hat{R}_{x, \cdot}^{0}-N_{x, \cdot}^{0}\right\|_{2}$ is $\Omega_{\mathbb{P}}\left(T \sqrt{D(\alpha, p)} / n^{3 / 2}\right)$. In other words, $\hat{R}^{0}$ inherites the separability property.

Step 4. Proposition 7 ensures that the statement derived in Step 3 holds. From there and using the result of Step 2, we conclude that the number of misclassified states satisfies Theorem 2.

Step 1. This is formalized in Lemma 4, and proven in §SM4.2. It is a consequence of the block structure of matrix $N$.

Lemma 4. For $x, y \in \mathcal{V}$ s.t. $\sigma(x) \neq \sigma(y),\left\|N_{x, \cdot}^{0}-N_{y, \cdot}^{0}\right\|_{2}=\Omega\left(\frac{T \sqrt{D(\alpha, p)}}{n^{3 / 2}}\right)$.

Step 2. Lemma 5 shows that the error $\left\|\hat{R}^{0}-N^{0}\right\|_{\mathrm{F}}$ is asymptotically bounded by $\left\|\hat{N}_{\Gamma}-N\right\|$, and is proven in $\S \mathrm{SM} 4.3$. The proof relies on a powerful bound relating decompositions of random matrices and their spectra [22].

LEMma 5. $\left\|\hat{R}^{0}-N^{0}\right\|_{\mathrm{F}} \leq \sqrt{16 K}\left\|\hat{N}_{\Gamma}-N\right\|$.

Step 3. The result obtained in this step is stated in Lemma 6. Its proof, presented in $\S \mathrm{SM} 4.4$, requires a bound on the spectral concentration rate of $\hat{N}_{\Gamma}$ 's noise matrix, relies on Lemmas 4,5 , and exploits the design of the $K$-means algorithm used after the spectral decomposition.

LEMMA 6. If $\left\|\hat{N}_{\Gamma}-N\right\|=o \mathbb{P}\left(f_{n}\right)$ for some sequence $f_{n}=o(T / n)$ and there exists a sequence $h_{n}$ such that $\omega\left(f_{n} / \sqrt{n}\right)=h_{n}=o\left(T \sqrt{D(\alpha, p)} / n^{3 / 2}\right)$, then

$$
\left\|\hat{R}_{x, \cdot}^{0}-N_{x, \cdot}^{0}\right\|_{2}=\Omega_{\mathbb{P}}\left(\frac{T D^{1 / 2}(\alpha, p)}{n^{3 / 2}}\right) \quad \text { for any misclassified state } \quad x \in \mathcal{E} .
$$

Step 4. In view of Proposition 7, the conditions of Lemma 6 are satisfied for e.g. $f_{n}=((T / n) \ln (T / n))^{1 / 2+\zeta}$ and $h_{n}=\left(f_{n} / \sqrt{n}\right)(T / n)^{\zeta}$ with $0<\zeta<1 / 4$. The final step is then almost immediate. Indeed, we have because of Lemma 6 $\left\|\hat{R}^{0}-N^{0}\right\|_{\mathrm{F}}^{2} \geq \sum_{x \in \mathcal{E}}\left\|\hat{R}_{x, \cdot}^{0}-N_{x, .}^{0}\right\|_{2}^{2}=|\mathcal{E}| \Omega_{\mathbb{P}}\left(T^{2} D(\alpha, p) / n^{3}\right)$. Hence, using Lemma $5,(1 /|\mathcal{E}|)\left\|\hat{N}_{\Gamma}-N\right\|^{2}=\Omega_{\mathbb{P}}\left(T^{2} D(\alpha, p) / n^{3}\right)$, or equivalently, since $I(\alpha, p)>0$ and hence $D(\alpha, p)>0,(n /|\mathcal{E}|)\left\|\hat{N}_{\Gamma}-N\right\|^{2}=\Omega_{\mathbb{P}}\left(T^{2} / n^{2}\right)$. We also have from Proposition 7 that $\left\|\hat{N}_{\Gamma}-N\right\|^{2}=O_{\mathbb{P}}\left(\frac{T}{n} \ln \frac{T}{n}\right)$. Combining the two previous equalities, we conclude that: $|\mathcal{E}| / n=O_{\mathbb{P}}\left(\frac{n}{T} \ln \frac{T}{n}\right)$. This is obtained by applying Lemma 22 presented in $\S$ SM6.5 (with $X_{n}=\left\|\hat{N}_{\Gamma}-N\right\|^{2}$, $Y_{n}=|\mathcal{E}| / n, y_{n}=T^{2} / n^{2}$, and $\left.x_{n}=(T / n) \ln (T / n)\right)$. 


\section{Performance of the Cluster Improvement Algorithm.}

7.1. Intuition behind the algorithm. Let us first briefly explain the intuition behind the Cluster Improvement Algorithm: given a cluster assignment, the algorithm inspects for each state the cluster assignment that makes the observed sample path the most likely. It then assigns the state accordingly.

Note that such a greedy assignment based on maximizing a likelihood function is generally not guaranteed to converge to a good minimum. Indeed; first, if the initial cluster assignment is far from the true assignment, the approximated BMC parameters $\hat{p}, \hat{\pi}$, and $\hat{\alpha}$ will also be far from the true parameters. The cluster improvement algorithm will then weigh the sample paths according to an incorrect model and may assign states to incorrect clusters. Second, the induced dependencies between consecutive updates of our improvement algorithm threaten our chances of deriving a performance upper bound that gets tighter with the number of iterations.

7.2. Proof of Theorem 3. Our proof tackles both concerns - the concerns of assigning vertices according to an incorrect model, and of having strong dependencies between consecutive updates - by considering the precise asymptotic concentration rates of the BMC. Tying the first concern to concentration is straightforward: as long as the initial cluster assignment provides sufficiently good estimates of the BMC parameters, the sample path will be weighted according to a model that is close to the ground truth. The method with which we overcome the second concern is more refined. Specifically, in the proof of Theorem 3, we split the set of all states into a set of well-behaved states $\mathcal{H}$ and a set of forlorn states $\mathcal{H}^{\mathrm{c}}$.

Definition. The set of well-behaved states $\mathcal{H}$ is the largest set of states $x \in \Gamma$ that satisfy the following two properties:

(H1) When $x \in \mathcal{V}_{i}$, for all $j \neq i$,

$$
\sum_{k=1}^{K}\left(\hat{N}_{x, \mathcal{V}_{k}} \ln \frac{p_{i, k}}{p_{j, k}}+\hat{N}_{\mathcal{V}_{k}, x} \ln \frac{p_{k, i} \alpha_{j}}{p_{k, j} \alpha_{i}}\right)+\left(\frac{\hat{N}_{\mathcal{V}_{j}, \mathcal{V}}}{\alpha_{j} n}-\frac{\hat{N}_{\mathcal{V}_{i}, \mathcal{V}}}{\alpha_{i} n}\right) \geq \frac{T}{2 n} I(\alpha, p) .
$$

(H2) $\hat{N}_{x, \mathcal{V} \backslash \mathcal{H}}+\hat{N}_{\mathcal{V} \backslash \mathcal{H}, x} \leq 2 \ln \left((T / n)^{2}\right)$.

States in $\mathcal{H}$ satisfy properties (H1) and (H2), which guarantee that these states will likely be assigned to their true cluster in our greedy repeated local maximization of the log-likelihood function. To be precise, the set is designed such that the cardinality of the intersection $\mathcal{E}_{\mathcal{H}}^{[t]} \triangleq \mathcal{E}^{[t]} \cap \mathcal{H}$ of incorrectly 
classified states and well-behaved states shrinks at each iteration with high probability. Furthermore, as we will show this set converges to the empty set with high probability after $t \approx \ln n$ iterations. Because we cannot guarantee that states in $\mathcal{H}^{\mathrm{c}}$ are classified correctly as the algorithm greedily allocates vertices to clusters, we simply treat all of these vertices as being misclassified - a worst-case upper bound. By then estimating the size of $\mathcal{H}^{\mathrm{c}}$, we are able to bound the total number of misclassified vertices after $t \in \mathbb{N}_{0}$ improvement steps of the Cluster Improvement Algorithm.

How we prove that $\left|\mathcal{E}_{\mathcal{H}}^{[t]}\right|$ shrinks. Proposition 8 quantifies how $\left|\mathcal{E}_{\mathcal{H}}^{[t]}\right|$ is reduced in a single iteration.

Proposition 8. If $I(\alpha, p)>0$ and $T=\omega(n)$, and $\left|\mathcal{E}_{\mathcal{H}}^{[t]}\right|=O_{\mathbb{P}}\left(e_{n}^{[t]}\right)$ for some $0<e_{n}^{[t]}=o\left(\frac{n}{\ln (T / n)}\right)$, then

$$
\left|\mathcal{E}_{\mathcal{H}}^{[t+1]}\right| \asymp_{\mathbb{P}} e_{n}^{[t+1]}=O\left(e_{n}^{[t]} \frac{n}{T} \ln \left(\frac{T}{n}\right)\right)=o\left(e_{n}^{[t]}\right) .
$$

To establish Proposition 8, observe that after the $(t+1)$-th iteration, for any misclassified state $x$, its true cluster $\sigma(x)$ does not maximize the objective function $u_{x}^{[t]}(c)$. Hence, summing over all misclassified states that also belong to $\mathcal{H}$, we obtain

$$
E \triangleq \sum_{x \in \mathcal{E}_{\mathcal{H}}^{[t+1]}}\left(u_{x}^{[t]}\left(\sigma^{[t+1]}(x)\right)-u_{x}^{[t]}(\sigma(x))\right) \geq 0 .
$$

We prove Proposition 8 by analyzing $E$. After substituting $u_{x}^{[t]}$ 's definition (18) into (35), we decompose $E$ as $E=E_{1}+E_{2}+U$, where

$$
\begin{aligned}
& E_{1}=\sum_{x \in \mathcal{E}_{\mathcal{H}}^{[t+1]}}\left\{\sum_{k=1}^{K}\left(\hat{N}_{x, \mathcal{V}_{k}} \ln \frac{p_{\sigma^{[t+1]}(x), k}}{p_{\sigma(x), k}}+\hat{N}_{\mathcal{V}_{k}, x} \ln \frac{p_{k, \sigma^{[t+1]}(x)} \alpha_{\sigma(x)}}{p_{k, \sigma(x)} \alpha_{\sigma}{ }^{[t+1]}(x)}\right)\right. \\
& \left.+\left(\frac{\hat{N}_{\mathcal{V}_{\sigma(x)}, \mathcal{V}}}{\alpha_{\sigma(x)} n}-\frac{\hat{N}_{\mathcal{V}_{\sigma^{[t+1]}(x)}}, \mathcal{V}}{\alpha_{\sigma^{[t+1]}(x)} n}\right)\right\} \\
& E_{2}=\sum_{x \in \mathcal{E}_{\mathcal{H}}^{[t+1]}} \sum_{k=1}^{K}\left(\left(\hat{N}_{x, \hat{\mathcal{V}}_{k}^{[t]}}-\hat{N}_{x, \mathcal{V}_{k}}\right) \ln \frac{p_{\sigma^{[t+1]}(x), k}}{p_{\sigma(x), k}}\right. \\
& \left.+\left(\hat{N}_{\hat{\mathcal{V}}_{k}^{[t]}, x}-\hat{N}_{\mathcal{V}_{k}, x}\right) \ln \frac{p_{k, \sigma}^{[t+1]}(x)}{p_{k, \sigma(x)}}\right),
\end{aligned}
$$

imsart-aos ver. 2014/10/16 file: JS_AP_SY__CBMC__arXiv.tex date: July 31, 2019 
Importantly, note that $E_{1}$ and $E_{2}$ account for the true model parameters $p$ and $\alpha$, whereas in the functions $u_{x}^{[t]}$ used in the algorithm, we replace these parameters by their estimates $\hat{p}$ and $\hat{\alpha}$. The term $U$ hence captures issues due to estimation errors. By construction of $\mathcal{H}, E_{1} \leq-(T / n) I(\alpha, p)\left|\mathcal{E}_{\mathcal{H}}^{[t+1]}\right|$. Using concentration results for the BMC, we can show that $E_{2} \approx \| \hat{N}_{\Gamma}-$ $N \|\left(\left|\mathcal{E}_{\mathcal{H}}^{[t+1]}\right|\left|\mathcal{E}_{\mathcal{H}}^{[t]}\right|\right)^{1 / 2}$ and that $U \approx 0$, asymptotically. These observations are formalized in Lemma 7, proved in §SM5.2.

Lemma 7. If $T=\omega(n),\left|\mathcal{E}_{\mathcal{H}}^{[t]}\right|=O_{\mathbb{P}}\left(e_{n}^{[t]}\right)$ for some $0<e_{n}^{[t]}=o\left(\frac{n}{\ln (T / n)}\right)$ and $\left|\mathcal{E}_{\mathcal{H}}^{[t+1]}\right| \asymp_{\mathbb{P}} e_{n}^{[t+1]}$, then:

$$
\begin{aligned}
& -E_{1}=\Omega_{\mathbb{P}}\left(I(\alpha, p) \frac{T}{n} e_{n}^{[t+1]}\right), \\
& |U|=O\left(e_{n}^{[t+1]} \sqrt{\frac{T}{n}} \ln \frac{T}{n}+e_{n}^{[t+1]} \frac{e_{n}^{[t]}}{n} \frac{T}{n} \ln \frac{T}{n}\right), \text { and } \\
& \left|E_{2}\right| \leq F_{1}+F_{2}+F_{3} \text { with } F_{1}=O_{\mathbb{P}}\left(\frac{T}{n} \frac{e_{n}^{[t]}}{n} e_{n}^{[t+1]}\right), \\
& F_{2}=O_{\mathbb{P}}\left(\sqrt{\frac{T}{n} \ln \left(\frac{T}{n}\right) e_{n}^{[t]} e_{n}^{[t+1]}}\right), \text { and } F_{3}=O_{\mathbb{P}}\left(\ln (T / n)^{2} e_{n}^{[t+1]}\right) .
\end{aligned}
$$

Combining Lemma 7 with the fact that $E \geq 0$ yields (34). Indeed, we have $-E_{1} \leq\left|E_{2}\right|+|U|$ almost surely. Now observe that since $\sqrt{\frac{T}{n}} \ln \frac{T}{n}=o\left(\frac{T}{n}\right)$ and by assumption $e_{n}^{[t]}=o(n / \ln (T / n)),|U|$ is negligible compared to $-E_{1}$, which implies that $-E_{1}-|U|=\Omega_{\mathbb{P}}\left(I(\alpha, p) \frac{T}{n} e_{n}^{[t+1]}\right)$. Similarly, since by assumption $e_{n}^{[t]}=o(n)$ and $\ln \left((T / n)^{2}\right)=o\left(\frac{T}{n}\right), F_{1}$ and $F_{3}$ are also negligible compared to $-E_{1}$, which implies that $-E_{1}-|U|-F_{1}-F_{3}=\Omega_{\mathbb{P}}\left(I(\alpha, p) \frac{T}{n} e_{n}^{[t+1]}\right)$. Finally, in view of Lemma $7 \Omega_{\mathbb{P}}\left(I(\alpha, p) \frac{T}{n} e_{n}^{[t+1]}\right)=-E_{1}-|U|-F_{1}-F_{3} \leq F_{2}=$ $O_{\mathbb{P}}\left(\sqrt{\frac{T}{n} \ln \frac{T}{n} e_{n}^{[t]} e_{n}^{[t+1]}}\right)$. We deduce that $I(\alpha, p) e_{n}^{[t+1]}=O\left(\sqrt{\frac{n}{T} \ln \frac{T}{n} e_{n}^{[t]} e_{n}^{[t+1]}}\right) ;$ see Lemma 25 for the precise justification. Since $I(\alpha, p)>0$, we obtain $e_{n}^{[t+1]}=O\left(e_{n}^{[t]} \frac{n}{T} \ln \frac{T}{n}\right)$, which concludes the proof of Proposition 8.

How we bound the size of $\mathcal{H}^{\mathrm{c}}$. Proposition 9 provides an upper bound of the number of states in $\mathcal{H}^{\mathrm{c}}$, and is proved in §SM5.1.

Proposition 9. If $I(\alpha, p)>0$ and $T=\omega(n)$, and $\left|\mathcal{E}_{\mathcal{H}}^{[t]}\right|=O_{\mathbb{P}}\left(e_{n}^{[t]}\right)$ for some $0<e_{n}^{[t]}=o(n)$, then $\left|\mathcal{E}_{\mathcal{H}^{\mathrm{c}}}^{[t]}\right| \leq\left|\mathcal{H}^{\mathrm{c}}\right|=O_{\mathbb{P}}\left(n \exp \left(-C \frac{T}{n} I(\alpha, p)\right)\right)$, where $C=\alpha_{\min }^{2} /\left(720 \eta^{3} \alpha_{\max }^{2}\right), \alpha_{\max }=\max _{i} \alpha_{i}$, and $\alpha_{\min }=\min _{i} \alpha_{i}$.

We now sketch the proof of Proposition 9. First note that the number of states not in $\Gamma$ (obtained after the trimming process) is negligible, i.e., $n \exp \left(-\frac{T}{n} \ln \frac{T}{n}\right)$. We then upper bound the number of states that do not

imsart-aos ver. 2014/10/16 file: JS_AP_SY_CBMC__arXiv.tex date: July 31, 2019 
satisfy (H1). Let $x \in \mathcal{V}_{i}$. If $x$ does not satisfy (H1), there exists $j \neq i$ such that $\hat{I}_{i, j}(x)<\frac{T}{2 n} I(\alpha, p)$, where

$$
\hat{I}_{i, j}(x) \triangleq \sum_{k=1}^{K}\left(\hat{N}_{x, \mathcal{V}_{k}} \ln \frac{p_{i, k}}{p_{j, k}}+\hat{N}_{\mathcal{V}_{k}, x} \ln \frac{p_{k, i} \alpha_{j}}{p_{k, j} \alpha_{i}}\right)+\left(\frac{\hat{N}_{\mathcal{V}_{j}, \mathcal{V}}}{\alpha_{j} n}-\frac{\hat{N}_{\mathcal{V}_{i}, \mathcal{V}}}{\alpha_{i} n}\right) .
$$

Observe that $\mathbb{E}\left[\hat{I}_{i, j}(x)\right]=\frac{T}{n} I_{i, j}(\alpha, p)$ where $I_{i, j}(\alpha, p)$ is the quantity involved in the definition of $I(\alpha, p)$; see (10). In particular, $\mathbb{E}\left[\hat{I}_{i, j}(x)\right] \geq \frac{T}{n} I(\alpha, p)$. Hence, $x$ does not satisfy (H1) implies that for some $j \neq i, \hat{I}_{i, j}(x)<\frac{T}{2 n} I(\alpha, p)$ and $\mathbb{E}\left[\hat{I}_{i, j}(x)\right] \geq \frac{T}{n} I(\alpha, p)$. Using concentration results for the BMC $\S \mathrm{SM} 1$, this event happens with probability at most $\exp \left(-C \frac{T}{n} I(\alpha, p)\right)$. We next deduce a bound on the expected number of states not satisfying (H1). From there, using Markov's inequality, we obtain that the number of states not satisfying (H1) does not exceed $n \exp \left(-C \frac{T}{n} I(\alpha, p)\right)$ with high probability. Note that the constant $C=\alpha_{\min }^{2} /\left(720 \eta^{3} \alpha_{\max }^{2}\right)$ stems from the precise application of concentration results.

We then complete the proof through the following argument. Consider the following iterative construction: start with the set $Z(0)$ of all states that do not satisfy (H1). The $t$-th iteration consists of adding to $Z(t-1)$ a state $v$ not satisfying (H2) written w.r.t. $Z(t-1)$, i.e., $\hat{N}_{v, Z(t-1)}+\hat{N}_{Z(t-1), v}>$ $2 \ln \left((T / n)^{2}\right)$. If such a state does not exist, the construction stops. Let $Z\left(t^{*}\right)$ be the final set: $t^{*}$ is the number of iterations before the construction stops. Then $Z\left(t^{*}\right)$ is such that for all $x \notin Z\left(t^{*}\right), x$ satisfies (H1) and (H2) written w.r.t. $Z\left(t^{*}\right)$. Hence by definition of $\mathcal{H}$, the size of $\mathcal{V} \backslash Z\left(t^{*}\right)$ is smaller than that of $\mathcal{H}$, and thus $\left|\mathcal{H}^{\mathrm{c}}\right| \leq\left|Z\left(t^{*}\right)\right|$. To get an upper bound on $\left|Z\left(t^{*}\right)\right|$, we just establish an upper bound on $t^{*}$ using concentration results (at each iteration $t$, a large number (specifically $2 \ln \left((T / n)^{2}\right)$ ) of observed transitions inside $Z(t)$ is added, which rapidly becomes impossible). In summary: $\left|\mathcal{H}^{\mathrm{c}}\right| \leq$ $\left|Z\left(t^{*}\right)\right| \leq t^{*}+|Z(0)| \leq t^{*}+n \exp \left(-C \frac{T}{n} I(\alpha, p)\right)$ with high probability.

Iterating the bound. If we initiate the Cluster Improvement Algorithm using the cluster assignment provided by the Spectral Clustering Algorithm when $T=\omega(n)$, from Theorem 2, we satisfy the initial condition $\left|\mathcal{E}_{\mathcal{H}}^{[0]}\right|=o_{\mathbb{P}}\left(\frac{n}{\ln (T / n)}\right)$ of Propositions 8, 9. Furthermore, since $\left|\mathcal{E}^{[t]}\right|=\left|\mathcal{E}_{\mathcal{H}}^{[t]}\right|+\left|\mathcal{E}_{\mathcal{H}^{c}}^{[t]}\right|$, we conclude by iterating the bound in (34) that after $t \in \mathbb{N}_{0}$ improvement steps the Cluster Improvement Algorithm misclassifies at most

$$
\left|\mathcal{E}^{[t]}\right|=O_{\mathbb{P}}\left(\mathrm{e}^{\ln n-t\left(\ln \frac{T}{n}-\ln \ln \frac{T}{n}\right)}+\mathrm{e}^{\ln n-\frac{\alpha_{\min }^{2}}{720 \eta^{3} \alpha_{\max }^{2}} \frac{T}{n} I(\alpha, p)}+\mathrm{e}^{\ln n-\frac{T}{n} \ln \frac{T}{n}}\right)
$$

states. This completes the proof of Theorem 3 .

imsart-aos ver. 2014/10/16 file: JS_AP_SY_CBMC__arXiv.tex date: July 31, 2019 
8. Acknowledgments. We would like to thank our anonymous referees: their careful reading and suggestions have led to improved revisions of this work. We also thank Pascal Lagerweij for having conducted the numerical experiment in Section 4.4.

\section{Bibliography.}

[1] P. W. Holland, K. B. Laskey, and S. Leinhardt. "Stochastic blockmodels: First steps". In: Social networks 5.2 (1983), pp. 109-137.

[2] D. A. Levin, Y. Peres, and E. L. Wilmer. Markov Chains and Mixing Times. en. American Mathematical Soc., 2009.

[3] R. S. Sutton and A. G. Barto. Introduction to Reinforcement Learning. 1st. Cambridge, MA, USA: MIT Press, 1998.

[4] S. P. Singh, T. Jaakkola, and M. I. Jordan. "Reinforcement Learning with Soft State Aggregation". In: Advances in Neural Information Processing Systems 7. Ed. by G. Tesauro, D. S. Touretzky, and T. K. Leen. MIT Press, 1995, pp. 361-368. URL: http://papers . nips.cc/paper/981-reinforcement-learning-with-soft-stateaggregation.pdf.

[5] R. Ortner. "Adaptive aggregation for reinforcement learning in average reward Markov decision processes". In: Annals OR 208.1 (2013), pp. 321-336.

[6] C. Gao, Z. Ma, A. Y. Zhang, and H. H. Zhou. "Achieving optimal misclassification proportion in stochastic block model". In: arXiv preprint arXiv:1505.03772 (2015).

[7] A. Decelle, F. Krzakala, C. Moore, and L. Zdeborová. "Inference and phase transitions in the detection of modules in sparse networks". In: Physical Review Letters 107.6 (2011), p. 065701.

[8] L. Massoulié. "Community detection thresholds and the weak Ramanujan property". In: Proceedings of the forty-sixth annual ACM symposium on Theory of computing. ACM. 2014, pp. 694-703.

[9] E. Mossel, J. Neeman, and A. Sly. "Reconstruction and estimation in the planted partition model". In: Probability Theory and Related Fields 162.3-4 (2015), pp. 431-461.

[10] S.-Y. Yun and A. Proutiere. "Community Detection via Random and Adaptive Sampling." In: COLT. 2014, pp. 138-175.

[11] S.-Y. Yun and A. Proutiere. "Optimal cluster recovery in the labeled stochastic block model". In: Advances in Neural Information Processing Systems. 2016, pp. 965-973. 
[12] S.-Y. Yun and A. Proutiere. "Accurate community detection in the stochastic block model via spectral algorithms". In: arXiv preprint arXiv:1412.7335 (2014).

[13] E. Abbe and C. Sandon. "Community detection in general stochastic block models: Fundamental limits and efficient algorithms for recovery". In: Foundations of Computer Science (FOCS), 2015 IEEE 56th Annual Symposium on. IEEE. 2015, pp. 670-688.

[14] E. Abbe and C. Sandon. "Recovering communities in the general stochastic block model without knowing the parameters". In: Advances in neural information processing systems. 2015, pp. 676-684.

[15] V. Jog and P.-L. Loh. "Information-theoretic bounds for exact recovery in weighted stochastic block models using the Renyi divergence". In: arXiv preprint arXiv:1509.06418 (2015).

[16] E. Mossel, J. Neeman, and A. Sly. "Consistency thresholds for the planted bisection model". In: Proceedings of the forty-seventh annual ACM symposium on Theory of computing. ACM. 2015, pp. 69-75.

[17] E. Abbe, A. S. Bandeira, and G. Hall. "Exact recovery in the stochastic block model". In: IEEE Transactions on Information Theory 62.1 (2016), pp. 471-487.

[18] B. Hajek, Y. Wu, and J. Xu. "Achieving exact cluster recovery threshold via semidefinite programming". In: IEEE Transactions on Information Theory 62.5 (2016), pp. 2788-2797.

[19] T. Lai and H. Robbins. "Asymptotically efficient adaptive allocation rules". In: Advances in Applied Mathematics 6.1 (1985), pp. 4 -22. DOI: https : // doi . org/10 . 1016/0196-8858(85 ) 90002-8. URL: http : / / www . sciencedirect . com/science/article/pii / 0196885885900028.

[20] S. Lloyd. "Least squares quantization in PCM". In: IEEE transactions on information theory 28.2 (1982), pp. 129-137.

[21] P. Bremaud. Markov Chains: Gibbs Fields, Monte Carlo Simulation, and Queues. en. Springer, May 1999.

[22] N. Halko, P. G. Martinsson, and J. A. Tropp. "Finding Structure with Randomness: Probabilistic Algorithms for Constructing Approximate Matrix Decompositions". In: SIAM Review 53.2 (2011), pp. 217-288. DOI: 10 . $1137 / 090771806$. URL: http : / / dx . doi .org/10 .1137/ 090771806.

[23] E. P. Wigner. "On the distribution of the roots of certain symmetric matrices". In: Annals of Mathematics (1958), pp. 325-327.

[24] T. Tao. Topics in random matrix theory. Vol. 132. American Mathematical Society Providence, RI, 2012. 
[25] J. A. Tropp et al. "An introduction to matrix concentration inequalities". In: Foundations and Trends® in Machine Learning 8.1-2 (2015), pp. $1-230$.

[26] W. Hochstättler, W. Kirsch, and S. Warzel. "Semicircle law for a matrix ensemble with dependent entries". In: Journal of Theoretical Probability 29.3 (2016), pp. 1047-1068.

[27] W. Kirsch and T. Kriecherbauer. "Sixty years of moments for random matrices". In: arXiv preprint arXiv:1612.06725 (2016).

[28] W. Kirsch and T. Kriecherbauer. "Semicircle law for generalized CurieWeiss matrix ensembles at subcritical temperature". In: arXiv preprint arXiv:1703.05183 (2017).

[29] C. Bordenave, P. Caputo, and D. Chafai. "Spectrum of large random reversible Markov chains: two examples". In: ALEA: Latin American Journal of Probability and Mathematical Statistics 7 (2010), pp. 41-64.

[30] C. Bordenave, P. Caputo, D. Chafai, et al. "Spectrum of large random reversible Markov chains: heavy-tailed weights on the complete graph". In: The Annals of Probability 39.4 (2011), pp. 1544-1590.

[31] U. Feige and E. Ofek. "Spectral techniques applied to sparse random graphs". In: Random Structures 8 Algorithms 27.2 (2005), pp. 251-275.

[32] D. Paulin. "Concentration inequalities for Markov chains by Marton couplings and spectral methods". In: Electron. J. Probab. 20 (2015), 32 pp. DOI: 10.1214/EJP.v20-4039. URL: https://doi.org/10.1214/ EJP. v20-4039.

[33] A. Dax. "From eigenvalues to singular values: a review". In: Advances in Pure Mathematics 3.09 (2013), p. 8.

[34] R. Vershynin. "Introduction to the non-asymptotic analysis of random matrices". In: arXiv preprint arXiv:1011.3027 (2010).

[35] R. H. Keshavan, A. Montanari, and S. Oh. "Matrix completion from a few entries". In: IEEE transactions on information theory 56.6 (2010), pp. 2980-2998. 


\section{SUPPLEMENTARY MATERIAL}

SM1. Concentration inequalities for BMCs. Recall the notation $\hat{N}_{\mathcal{A}, \mathcal{B}}=\sum_{x \in \mathcal{A}} \sum_{y \in \mathcal{B}} \hat{N}_{x, y}$ for any subsets $\mathcal{A}, \mathcal{B} \subseteq \mathcal{V}$.

Proposition 10. The following concentration inequalities hold for BMCs for $T / n$ large enough (larger than a constant that does not depend on $n$, but on the BMC parameters):

- There exists an absolute constant $c_{1}>0$ such that for $k=1, \ldots, K$,

$$
\mathbb{P}\left[\left|\hat{N}_{\mathcal{V}, \mathcal{V}_{k}}-N_{\mathcal{V}, \mathcal{V}_{k}}\right| \geq c_{1} \sqrt{T \ln \frac{T}{n}}\right] \leq \frac{n^{2}}{T^{2}} .
$$

- There exists an absolute constant $c_{2}>0$ such that for any $x \in \mathcal{V}$,

$$
\begin{gathered}
\mathbb{P}\left[\left|\hat{N}_{\mathcal{V}, x}-N_{\mathcal{V}, x}\right| \geq c_{2} \frac{T}{n} \ln \frac{T}{n}\right] \leq \mathrm{e}^{-2 \frac{T}{n} \ln \frac{T}{n}}, \\
\mathbb{P}\left[\left|\hat{N}_{\mathcal{V}, x}-N_{\mathcal{V}, x}\right| \geq c_{2} \sqrt{\frac{T}{n}} \ln n\right] \leq \frac{1}{n^{2}} .
\end{gathered}
$$

- There exists an absolute constant $d_{3}>0$ such that for any $c_{3} \geq 1$ and any subset $\mathcal{S} \subset \mathcal{V}$ of size $|\mathcal{S}|=\lfloor n \exp (-(T / n) \ln (T / n))\rfloor$

$$
\mathbb{P}\left[\left|\hat{N}_{\mathcal{V}, \mathcal{S}}-N_{\mathcal{V}, \mathcal{S}}\right| \geq c_{3} n\right] \leq \mathrm{e}^{-d_{3} c_{3} n} .
$$

- There exists an absolute constant $c_{4}>0$ such that for any $i, j \in\{1, \ldots, K\}$, and $x \in \mathcal{V}_{i}$,

$$
\mathbb{P}\left[\sum_{t=1}^{T} f\left(\tilde{X}_{t}\right) \leq \frac{T}{2 n} I(\alpha, p)\right] \leq \mathrm{e}^{-c_{4} \frac{T}{n} I(\alpha, p)}
$$

where $\tilde{X}_{t}=\left(X_{t}, X_{t+1}\right)$ and

$$
\begin{aligned}
f\left(\tilde{X}_{t}\right)= & \sum_{k=1}^{K}\left(\mathbb{1}\left[X_{t-1}=x, X_{t} \in \mathcal{V}_{k}\right] \ln \frac{p_{i, k}}{p_{j, k}}+\mathbb{1}\left[X_{t-1} \in \mathcal{V}_{k}, X_{t}=x\right] \ln \frac{p_{k, i} \alpha_{j}}{p_{k, j} \alpha_{i}}\right) \\
& +\frac{1}{n}\left(\frac{\mathbb{1}\left[X_{t-1} \in \mathcal{V}_{j}\right]}{\alpha_{j}}-\frac{\mathbb{1}\left[X_{t-1} \in \mathcal{V}_{i}\right]}{\alpha_{i}}\right) .
\end{aligned}
$$

The constant $c_{4}$ can be chosen as $c_{4}=\alpha_{\min }^{2} /\left(720 \eta^{3} \alpha_{\max }^{2}\right)$.

- There exists an absolute constant $c_{5}>0$ such that

$$
\max _{y \in \Gamma}\left\{\hat{N}_{\Gamma, y} \vee \hat{N}_{y, \Gamma}\right\} \leq c_{5} \frac{T}{n} \ln \frac{T}{n} \quad \text { with probability } \quad 1-n \mathrm{e}^{-\frac{T}{n} \ln \frac{T}{n}} .
$$

- There exists an absolute constant $c_{6}>0$ such that

$$
\max _{\mathcal{A}, \mathcal{B} \subset \mathcal{V}}\left|\hat{N}_{\mathcal{A}, \mathcal{B}}-N_{\mathcal{A}, \mathcal{B}}\right|<c_{6} \sqrt{n T} \text { with probability } 1-\mathrm{e}^{-(4-\ln 2) n} .
$$

imsart-aos ver. 2014/10/16 file: JS_AP_SY__CBMC__arXiv.tex date: July 31, 2019 
The concentration inequalities in Proposition 10 can all be shown using [32, Thm. 3.4], which we reproduce here for your convenience. Theorem 4 concerns a (non-reversible) stationary Markov chain $\left\{X_{t}\right\}_{t \geq 0}$ with state space $\Omega$ and stationary distribution $\Pi$. Its pseudo spectral gap $\gamma_{\mathrm{ps}}$ is defined in [32, Eq. (3.3)], and we give it below the theorem.

Theorem 4 (Paulin, 2015). Let $f \in L^{2}(\Pi)$ with $\left|f(x)-\mathbb{E}_{\Pi}(f)\right| \leq C$ for every $x \in \Omega$ (for some constant $C>0$ ). Let $V_{f}$ be the variance of $f(X)$ when $X$ follows the stationary distribution $\Pi$. Then, for any $z>0$,

$$
\mathbb{P}_{\Pi}\left[\left|\sum_{t=1}^{T} f\left(X_{t}\right)-\mathbb{E}_{\Pi}\left[\sum_{t=1}^{T} f\left(X_{t}\right)\right]\right| \geq z\right] \leq 2 \exp \left(-\frac{z^{2} \gamma_{p s}}{8\left(T+1 / \gamma_{p s}\right) V_{f}+20 z C}\right) .
$$

In order to apply Theorem 4, we also use [32, Prop. 3.4], which relates the pseudo spectral gap to the mixing time of the Markov chain: for any $\varepsilon \in[0,1)$,

$$
\gamma_{\mathrm{ps}} \triangleq \max _{i \geq 1} \frac{1-\lambda\left(\left(P^{*}\right)^{i} P^{i}\right)}{i} \geq \frac{1-\varepsilon}{t_{\operatorname{mix}}(\varepsilon / 2)} \quad \text { with } \quad P^{*}(x, y) \triangleq \frac{P(x, y)}{\Pi(x)} \Pi(y) .
$$

Next it is important to note that Proposition 2 also holds for the Markov chain induced by the transitions of the BMC. To see this, define $\tilde{X}_{t} \triangleq\left(X_{t-1}, X_{t}\right)$ such that $\left\{\tilde{X}_{t}\right\}_{t \geq 0}$ denotes a Markov process describing the transitions of $\left\{X_{t}\right\}_{t \geq 0}$. Let $\tilde{P}$ and $\tilde{\Pi}$ be the transition kernel and the stationary distribution of $\tilde{X}_{t}$, respectively. Note now that $d_{\mathrm{TV}}\left(P_{x, \cdot}^{t}, \Pi\right)=d_{\mathrm{TV}}\left(\tilde{P}_{(x, y),}^{t+1}, \tilde{\Pi}\right)$ for all $x, y \in \mathcal{V}, t \in \mathbb{N}_{+}$. The mixing time of $\left\{\tilde{X}_{t}\right\}_{t \geq 0}$, therefore, requires one more transition than the mixing time of $\left\{X_{t}\right\}_{t \geq 0}$. As a consequence of Proposition 2, more precisely from (65), we have for both of our Markov processes $\left\{X_{t}\right\}_{t \geq 0}$, $\left\{\tilde{X}_{t}\right\}_{t \geq 0}$ that

$$
\gamma_{\mathrm{ps}} \geq \frac{1}{2\left(t_{\mathrm{mix}}(1 / 4)+1\right)} \geq \frac{1}{2(4 \eta+1)} .
$$

The last inequality is obtained by observing that $t_{\text {mix }}(1 / 4) \leq-\ln (4) / \ln (1-$ $1 / 2 \eta) \leq 2 \eta \ln (4) \leq 4 \eta$.

Proof of (39). Apply Theorem 4 with $f(x)=\mathbb{1}\left[x \in \mathcal{V}_{k}\right]$. Hence $\sum_{t=1}^{T} f\left(X_{t}\right)$ $=\hat{N}_{\mathcal{V}, \mathcal{V}_{k}}, C \leq 1$, and $V_{f} \leq \pi_{k}$. Then, for any constant $c>0$,

$$
\mathbb{P}\left[\left|\hat{N}_{\mathcal{V}, \mathcal{V}_{k}}-N_{\mathcal{V}, \mathcal{V}_{k}}\right| \geq c \sqrt{T \ln \frac{T}{n}}\right] \leq 2 \exp \left(-\frac{c^{2}}{16(4 \eta+1)} \ln \frac{T}{n}(1+o(1))\right) .
$$

imsart-aos ver. 2014/10/16 file: JS_AP_SY__CBMC_-arXiv.tex date: July 31, 2019 
The desired inequality is obtained by choosing, in the above inequality, $c=c_{1}$ such that $c_{1}^{2} \geq 32(4 \eta+1)$.

Proof of (40). Let $f(\cdot)=\mathbb{1}[\cdot=x]$ such that $\sum_{t=1}^{T} f\left(X_{t}\right)=\hat{N}_{\mathcal{V}, x}, C \leq 1$, and $V_{f} \leq \pi_{k} / \alpha_{k} n$. From Theorem 4 , it follows that for any constant $c>0$

$$
\mathbb{P}\left[\left|\hat{N}_{\mathcal{V}, x}-N_{\mathcal{V}, x}\right| \geq c \frac{T}{n} \ln \frac{T}{n}\right] \leq 2 \exp \left(-\frac{c}{40(4 \eta+1)} \frac{T}{n} \ln \frac{T}{n}(1+o(1))\right) .
$$

The desired inequality is obtained by choosing, in the above inequality, $c=c_{2}$ such that $c_{2} \geq 40(4 \eta+1)$.

Proof of (41). We use the same function $f$ as that used in the proof of (40). Theorem 4 yields: for any $c>0$,

$$
\mathbb{P}\left[\left|\hat{N}_{\mathcal{V}, x}-N_{\mathcal{V}, x}\right| \geq c \sqrt{\frac{T}{n}} \ln n\right] \leq 2 \exp \left(-\frac{c^{2} \frac{T}{n}(\ln n)^{2} \gamma_{\mathrm{ps}}}{\frac{8 T}{\alpha_{\min } n}+20 c \sqrt{\frac{T}{n}} \ln n}(1+o(1))\right) .
$$

If $8 T /\left(n \alpha_{\min }\right) \geq 20 c \sqrt{T / n} \ln n$, the r.h.s. in (51) is less than $2 \exp \left(-\frac{c^{2} \alpha_{\min }}{32(4 \eta+1)}\right.$ $\left.(\ln n)^{2}(1+o(1))\right)$. If $8 T /\left(n \alpha_{\min }\right)<20 c \sqrt{T / n} \ln n$, the r.h.s. in (51) is smaller than $2 \exp \left(-\frac{c}{80(4 \eta+1)}(\ln n)(1+o(1))\right.$. The desired inequality is obtained by choosing $c=c_{2}>\max \left\{\sqrt{32(4 \eta+1) / \alpha_{\min }}, 160(4 \eta+1)\right\}$.

Proof of (42). Let $\mathcal{S} \subset \mathcal{V}$ be such that $|S|=\left\lfloor n \exp \left(-\frac{T}{n} \ln \frac{T}{n}\right)\right\rfloor$. Let $f(\cdot)=$ $\mathbb{1}[\cdot \in \mathcal{S}]$ such that $\sum_{t=1}^{T} f\left(X_{t}\right)=\hat{N}_{\mathcal{V}, S}, C \leq 1$, and $V_{f}=O\left(\exp \left(-\frac{T}{n} \ln \frac{T}{n}\right)\right)$. Theorem 4 implies that for any constant $c_{3}>0$,

$$
\mathbb{P}\left[\left|\hat{N}_{\mathcal{V}, \mathcal{S}}-N_{\mathcal{V}, \mathcal{S}}\right| \geq c_{3} n\right] \leq 2 \exp \left(-\frac{c_{3}}{40(4 \eta+1)} n(1+o(1))\right) .
$$

The result is proved for $d_{3}=1 /(40(4 \eta+1))$.

Proof of (43). We apply Theorem 4 to the Markov chain $\left\{\tilde{X}_{t}\right\}_{t \geq 0}$ and the function $f$ defined in (43) for some $i, j$ and $x \in \mathcal{V}_{i}$. Observe that we have $\mathbb{E}_{\Pi}\left[f\left(\tilde{X}_{t}\right)\right]=(1 / n) I_{i, j}(\alpha, p)$ by definition of $f$, where $I_{i, j}(\alpha, p)$ has been introduced when defining $I(\alpha, p)$ in (10).

When applying Theorem 4 , one can easily check that

$$
C \leq \frac{1}{n} I_{i, j}(\alpha, p)+\ln \frac{\eta \alpha_{\max }}{\alpha_{\min }}+\frac{1}{\alpha_{\min } n} .
$$

Let us analyze $V_{f}$. Since $\operatorname{Var}\left(f\left(\tilde{X}_{t}\right)\right) \leq \mathbb{E}\left[f\left(\tilde{X}_{t}\right)^{2}\right]$,

$$
V_{f} \leq \sum_{k=1}^{K} \frac{1}{\alpha_{i} n}\left(\pi_{i} p_{i, k}\left(\ln \frac{p_{i, k}}{p_{j, k}}\right)^{2}+\pi_{k} p_{k, i}\left(\ln \frac{p_{k, i} \alpha_{j}}{p_{k, j} \alpha_{i}}\right)^{2}\right)+O\left(\frac{1}{n^{2}}\right) .
$$

imsart-aos ver. 2014/10/16 file: JS_AP_SY__CBMC_-arXiv.tex date: July 31, 2019 
Now we can express $I_{i, j}(\alpha, p)$ using KL divergences, and show that:

$$
I_{i, j}(\alpha, p)=\frac{\pi_{i}}{\alpha_{i}} \operatorname{KL}\left(p_{i, .} \| p_{j, .}\right)+n \mathrm{KL}\left(\tilde{p}_{\cdot, i} \| \tilde{p}_{\cdot, j}\right)+O\left(\frac{1}{n}\right),
$$

where $\tilde{p}_{\cdot, i} \triangleq\left[\pi_{1} \frac{p_{1, i}}{\alpha_{i} n}, \pi_{1}\left(1-\frac{p_{1, i}}{\alpha_{i} n}\right), \ldots, \pi_{K} \frac{p_{K, i}}{\alpha_{i} n}, \pi_{K}\left(1-\frac{p_{K, i}}{\alpha_{i} n}\right)\right]$ and we have used the fact that $|\ln (1+x)-x| \leq x^{2}$ for $x \in[-1 / 2,1 / 2]$. Using $\tilde{p}_{\cdot, i}$, (54) is reformulated as follows:

$$
V_{f} \leq \frac{\pi_{i}}{\alpha_{i} n} \sum_{k=1}^{K} p_{i, k}\left(\ln \frac{p_{i, k}}{p_{j, k}}\right)^{2}+\sum_{k^{\prime}=1}^{2 K} \tilde{p}_{k^{\prime}, i}\left(\ln \frac{\tilde{p}_{k^{\prime}, i}}{\tilde{p}_{k^{\prime}, j}}\right)^{2}+O\left(\frac{1}{n^{2}}\right) .
$$

Next, in view of (55), Lemma 19 in $§ S M 6.3$ allows us to upper bound the r.h.s. of (56) using $I_{i, j}(\alpha, p)$. Specifically, we deduce that:

$$
V_{f} \leq\left(\eta \frac{\alpha_{\max }}{\alpha_{\min }}\right)^{2} \frac{1}{n} I_{i, j}(\alpha, p)+O\left(\frac{1}{n^{2}}\right) .
$$

Putting (48), (53), and (57) into Theorem 4, we have

$$
\begin{aligned}
& \mathbb{P}\left[\left|\sum_{t=1}^{T} f\left(\tilde{X}_{t}\right)-\mathbb{E}_{\pi}\left(f\left(\tilde{X}_{t}\right)\right)\right| \geq \frac{T}{2 n} I_{i, j}(\alpha, p)\right] \\
(58) & \leq 2 \exp \left(-\frac{1}{16(4 \eta+1)\left(4\left(\frac{\eta \alpha_{\max }}{\alpha_{\min }}\right)^{2}+5 \ln \frac{\eta \alpha_{\max }}{\alpha_{\min }}\right)} \frac{T}{n} I_{i, j}(\alpha, p)(1+o(1))\right) .
\end{aligned}
$$

Note that (43) is directly deduced from (58) and the fact that $I_{i, j}(\alpha, p) \geq$ $I(\alpha, p)$. The constant $c_{4}$ can be chosen as $c_{4}=\alpha_{\min }^{2} /\left(720 \eta^{3} \alpha_{\max }^{2}\right)$ (observe that since $\left.\eta \geq 1, \ln \left(\eta \alpha_{\max } / \alpha_{\min }\right) \leq\left(\eta \alpha_{\max } / \alpha_{\min }\right)^{2}\right)$.

Proof of (44). This concentration result is a direct consequence of (40).

Proof of (45). Consider any sets $\mathcal{A}, \mathcal{B} \subset \mathcal{V}$ and let $f\left(\tilde{X}_{t}\right)=\mathbb{1}\left[X_{t-1} \in\right.$ $\left.\mathcal{A}, X_{t} \in \mathcal{B}\right]$. For this function, $C \leq 1$ and $V_{f}=O(1)$. It follows from Theorem 4 that for any $c>0$ :

$$
\mathbb{P}\left[\left|\hat{N}_{\mathcal{A}, \mathcal{B}}-N_{\mathcal{A}, \mathcal{B}}\right| \geq c \sqrt{n T}\right] \leq 2 \exp \left(-\frac{c^{2}}{16(4 \eta+1)} n(1+o(1))\right) .
$$

The result is obtained by selecting $c=c_{6}$ such that $\frac{c_{6}^{2}}{16(4 \eta+1)} \geq 4$, and by applying a union bound over all possible subsets of $\mathcal{V}$ (there are $2^{n}$ such subsets).

\section{SM2. Proofs of Chapter 2.}

imsart-aos ver. 2014/10/16 file: JS_AP_SY_CBMC__arXiv.tex date: July 31, 2019 


\section{SM2.1. Proof of Proposition 1.}

Proof. We first prove that $\pi$ is a probability distribution. This follows by (i) definition of $\pi$, (ii) symmetry of all states in the same cluster, and (iii) because $\Pi$ is a probability distribution:

$$
\sum_{k=1}^{K} \pi_{k} \stackrel{(\mathrm{i})}{=} \sum_{k=1}^{K} \lim _{n \rightarrow \infty} \bar{\Pi}_{k}\left|\mathcal{V}_{k}\right| \stackrel{(\mathrm{ii})}{=} \lim _{n \rightarrow \infty} \sum_{k=1}^{K} \sum_{x \in \mathcal{V}_{k}} \Pi_{x}=\lim _{n \rightarrow \infty} \sum_{x \in \mathcal{V}} \Pi_{x} \stackrel{(\mathrm{iii})}{=} 1 .
$$

Next, we show that the balance equations hold. For $k=1, \ldots, K$ it follows by symmetry of any two states $x, z \in \mathcal{V}_{k}$ that $\Pi_{x}=\Pi_{z}=\bar{\Pi}_{k}$. Hence for any $y \in \mathcal{V}_{l}$, by (iv) global balance

$$
\Pi_{y}=\bar{\Pi}_{l} \stackrel{(\mathrm{iv})}{=} \sum_{k=1}^{K} \sum_{x \in \mathcal{V}_{k}} \Pi_{x} P_{x, y}=\sum_{k=1}^{K} \bar{\Pi}_{k}\left(\left|\mathcal{V}_{k}\right|-\mathbb{1}[k=l]\right) \frac{p_{k, l}}{\left|\mathcal{V}_{l}\right|-\mathbb{1}[k=l]}
$$

Letting $n \rightarrow \infty$, we find that $\pi_{l}=\sum_{k=1}^{K} \pi_{k} p_{k, l}$ for all $k, l$. This completes the proof.

\section{SM2.2. Proof of Proposition 2.}

Proof. We will use Dobrushin's ergodic coefficient, which is defined for any stochastic matrix $P$ by $[21$, Definition 7.1$]$

$$
\delta(P) \triangleq \frac{1}{2} \sup _{x, y \in \mathcal{V}} \sum_{z \in \mathcal{V}}\left|P_{x, z}-P_{y, z}\right|
$$

Moreover, Dobrushin's coefficient satisfies $\delta(P)=1-\inf _{x, y \in \mathcal{V}} \sum_{z \in \mathcal{V}}\left(P_{x, z} \wedge\right.$ $\left.P_{y, z}\right)\left[21\right.$, Eq. (7.3)]. Now recall our assumption $\exists_{0<\eta \neq 1}: \max _{a, b, c}\left\{p_{b, a} / p_{c, a}\right.$, $\left.p_{a, b} / p_{a, c}\right\} \leq \eta$, which implies that

$$
\begin{aligned}
\delta(P) & <1-\inf _{x, y \in \mathcal{V}} \sum_{z \in \mathcal{V}}\left(P_{x, z} \wedge P_{y, z}\right) \\
& \leq 1-\inf _{x \in \mathcal{V}} \sum_{z \in \mathcal{V}} \frac{1}{\eta} P_{x, z} \frac{\alpha_{\min } n-2}{\alpha_{\min } n}=1-\frac{1}{\eta} \frac{\alpha_{\min } n-2}{\alpha_{\min } n} .
\end{aligned}
$$

We deduce that when $n \geq 4 / \alpha_{\min }, \delta(P)<1-1 /(2 \eta)$ (recall that $\eta>1$ by definition).

Next [21, Thm. 7.2] gives us the convergence rate in terms of Dobrushin's coefficient. Specifically,

$$
d_{\mathrm{TV}}\left(P_{x, \cdot}^{t}, \Pi\right) \leq(\delta(P))^{t} d_{\mathrm{TV}}\left(P_{x, \cdot}^{0}, \Pi\right) \quad \text { for } \quad x \in \mathcal{V} .
$$

imsart-aos ver. 2014/10/16 file: JS_AP_SY__CBMC__arXiv.tex date: July 31, 2019 
As a consequence

$$
d_{\mathrm{TV}}\left(P_{x, \cdot}^{t}, \Pi\right) \leq \varepsilon \quad \text { whenever } \quad t \geq \frac{\ln \varepsilon}{\ln \left(1-\frac{1}{2 \eta}\right)}
$$

Therefore,

$$
t_{\text {mix }}(\varepsilon) \leq \frac{\ln \varepsilon}{\ln \left(1-\frac{1}{2 \eta}\right)}
$$

This completes the proof.

\section{SM3. Proofs of Chapter 5.}

SM3.1. $Q$ is a stochastic matrix. Observe that for $x \in \mathcal{V} \backslash\left\{V^{*}\right\}$,

$$
\begin{aligned}
& \sum_{y \in \mathcal{V}} Q_{x, y}=\frac{q_{\omega(x), 0}}{n}+\sum_{y \in \mathcal{W}_{\omega(x)} \backslash\{x\}} \frac{q_{\omega(x), \omega(x)}}{\left|\mathcal{W}_{\omega(x)}\right|-1}+\sum_{k=1}^{K} \mathbb{1}[k \neq \omega(x)] \sum_{y \in \mathcal{W}_{k}} \frac{q_{\omega(x), k}}{\left|\mathcal{W}_{k}\right|} \\
& (66) \quad=\frac{q_{\omega(x), 0}}{n}+\sum_{k=1}^{K} q_{\omega(x), k} \stackrel{(24)}{=} \frac{q_{\omega(x), 0}}{n}+\sum_{k=1}^{K}\left(p_{\omega(x), k}-\frac{q_{\omega(x), 0}}{K n}\right)=1 .
\end{aligned}
$$

Similarly for $x=V^{*}: \sum_{y \in \mathcal{V}} Q_{V^{*}, y}=\sum_{k=1}^{K} \sum_{y \in \mathcal{W}_{k}} \frac{q_{0, k}}{\left|\mathcal{W}_{k}\right|}=\sum_{k=1}^{K} q_{0, k}=1$.

SM3.2. Proof of Proposition 3. We first show that $\left(\gamma_{0}^{[0]}, \gamma_{1}^{[0]}, \ldots, \gamma_{K}^{[0]}\right)$ is a probability distribution. We have:

$$
\begin{aligned}
\gamma_{0}^{[0]}+\sum_{k=1}^{K} \gamma_{k}^{[0]} & =\lim _{n \rightarrow \infty}\left(\sum_{k=1}^{K}\left|\mathcal{W}_{k}\right| \bar{\Pi}_{k}^{(Q)}+\Pi_{V^{*}}^{(Q)}\right)=\lim _{n \rightarrow \infty}\left(\sum_{k=1}^{K} \sum_{x \in \mathcal{W}_{k}} \Pi_{x}^{(Q)}+\Pi_{V^{*}}^{(Q)}\right) \\
& =\lim _{n \rightarrow \infty} \sum_{x \in \mathcal{V}} \Pi_{x}^{(Q)}=1,
\end{aligned}
$$

where the last equality stems from the fact that $\Pi^{(Q)}$ is a probability distribution. Next, we have:

$$
\begin{aligned}
\gamma_{0}^{[0]} & =\lim _{n \rightarrow \infty} \Pi_{V^{*}}^{(Q)}=\lim _{n \rightarrow \infty} \sum_{x \in \mathcal{V}} \Pi_{x}^{(Q)} Q_{x, V^{*}} \stackrel{(25)}{=} \lim _{n \rightarrow \infty} \sum_{k=1}^{K} \sum_{x \in \mathcal{W}_{k}} \bar{\Pi}_{k}^{(Q)} \frac{q_{k, 0}}{n} \\
& =\lim _{n \rightarrow \infty} \sum_{k=1}^{K} \gamma_{k}^{[0]} \frac{q_{k, 0}}{n}=0,
\end{aligned}
$$


where the second equality stems from the global balance equations for $\Pi^{(Q)}$. Now we establish that the vector $\left(\gamma_{1}^{[0]}, \ldots, \gamma_{K}^{[0]}\right)^{\mathrm{T}}$ satisfies the balance equations $\left(\gamma_{1}^{[0]}, \ldots, \gamma_{K}^{[0]}\right) p=\left(\gamma_{1}^{[0]}, \ldots, \gamma_{K}^{[0]}\right)$. For $l=1, \ldots, K$

$$
\begin{aligned}
\gamma_{l}^{[0]} & =\lim _{n \rightarrow \infty}\left|\mathcal{W}_{l}\right| \bar{\Pi}_{l}^{(Q)}=\lim _{n \rightarrow \infty} \sum_{y \in \mathcal{W}_{l}} \Pi_{y}^{(Q)} \stackrel{(i i)}{=} \lim _{n \rightarrow \infty} \sum_{y \in \mathcal{W}_{l}} \sum_{x \in \mathcal{V}} \Pi_{x}^{(Q)} Q_{x, y} \\
& \stackrel{(25)}{=} \lim _{n \rightarrow \infty} \sum_{y \in \mathcal{W}_{l}}\left(\sum_{k=1}^{K} \sum_{x \in \mathcal{W}_{k} \backslash\{y\}} \bar{\Pi}_{k}^{(Q)} \frac{q_{k, l}}{\left|\mathcal{W}_{l}\right|-\mathbb{1}[k=l]}+\Pi_{V^{*}}^{(Q)} \frac{q_{0, l}}{\left|\mathcal{W}_{l}\right|}\right) \\
& =\lim _{n \rightarrow \infty}\left(\sum_{k=1}^{K}\left(\left|\mathcal{W}_{k}\right|-\mathbb{1}[k=l]\right) \bar{\Pi}_{k}^{(Q)} \frac{\left|\mathcal{W}_{l}\right|}{\left|\mathcal{W}_{l}\right|-\mathbb{1}[k=l]} q_{k, l}+\Pi_{V^{*}}^{(Q)} q_{0, l}\right) \\
& \stackrel{(24)}{=} \sum_{k=1}^{K} \gamma_{k}^{[0]} p_{k, l} .
\end{aligned}
$$

This proves the first two assertions of the proposition. The proof of the third assertion is similar to that of (68). More precisely:

$$
\begin{aligned}
\gamma_{0}^{[1]} & =\lim _{n \rightarrow \infty} n \Pi_{V^{*}}^{(Q)}=\lim _{n \rightarrow \infty} n \sum_{x \in \mathcal{V}} \Pi_{x}^{(Q)} Q_{x, V^{*}} \stackrel{(25)}{=} \lim _{n \rightarrow \infty} n \sum_{k=1}^{K} \sum_{x \in \mathcal{W}_{k}} \bar{\Pi}_{k}^{(Q)} \frac{q_{k, 0}}{n} \\
(70) & =\sum_{k=1}^{K} \gamma_{k}^{[0]} q_{k, 0} .
\end{aligned}
$$

Together with the first assertion, this completes the proof.

SM3.3. Proof of Lemma 2. Define for $c \in\{1, \ldots, K\}: q_{c}=\left(0, p_{1, c} / \alpha_{c}\right.$, $\left.\ldots, p_{K, c} / \alpha_{c} ; 0, p_{c, 1}, \ldots, p_{c, K}\right) \in \mathcal{Q}$. Let $a \neq b$. The points $q_{a}, q_{b}$ satisfy: $I_{a}\left(q_{a} \| p\right)=I_{b}\left(q_{b} \| p\right)=0$, and $0<I(\alpha, p) \leq I_{a}\left(q_{b} \| p\right)<\infty$ and $0<I(\alpha, p) \leq$ $I_{b}\left(q_{a} \| p\right)<\infty$ (by definition of $I(\alpha, p)$ ). Consider the function $g:[0,1] \mapsto \mathbb{R}$ defined by $g(\lambda)=I_{a}\left(\lambda q_{a}+(1-\lambda) q_{b} \| p\right)-I_{b}\left(\lambda q_{a}+(1-\lambda) q_{b} \| p\right)$. This function $g$ is continuous and from the aforementioned properties of $q_{a}$ and $q_{b}$, we have $g(0)>0$ and $g(1)<0$. The intermediate value theorem implies that there exists $\lambda \in(0,1)$ such that $I_{a}\left(\lambda q_{a}+(1-\lambda) q_{b} \| p\right)=I_{b}\left(\lambda q_{a}+(1-\lambda) q_{b} \| p\right)$. Hence $\mathcal{Q}(a, b) \neq \emptyset$.

\section{SM3.4. Proof of Proposition 4.}

Proof of Proposition 4 (i). Consider a fixed $n$. We have a BMC with true parameters $\left\{p_{k, l}\right\}$, and $\left\{\left|\mathcal{V}_{k}\right|\right\}$. We pick $V^{*}$ uniformly at random from clusters $a \neq b$ and construct $Q$. Recall that $Q$ is therefore random and depends on $V^{*},\left\{p_{k, l}\right\}$, and $\left\{\left|\mathcal{V}_{k}\right|\right\}$. We now give a lower bound on the probability that a

imsart-aos ver. 2014/10/16 file: JS_AP_SY_CBMC__arXiv.tex date: July 31, 2019 
$(\varepsilon, 1)$-locally good algorithm at $(\alpha, p)$ that does not have access to $\left\{p_{k, l}\right\}$ and $\left\{\left|\mathcal{V}_{k}\right|\right\}$ misclassifies $V^{*}$.

Consider such algorithm. Suppose we give the algorithm an infinitely long sample path generated under $Q$, i.e., $T \rightarrow \infty$. As a consequence, this algorithm has access to the elements $Q_{x, y}$ for all $x, y \in \mathcal{V}$ (from this it can determine which state is $V^{*}$ ). We furthermore give the algorithm the information that the two clusters from which we randomly picked are $a, b$. The algorithm must now consider the following two hypotheses:

$-\mathcal{H}_{a}=\left\{\hat{\sigma}\left(V^{*}\right)=a, \hat{p}_{\sigma(x), \sigma(y)}=\left(\left|\mathcal{V}_{\sigma(y)}\right|-\mathbb{1}[\sigma(y)=a]-\mathbb{1}\left[x \neq V^{*}, \sigma(x)=\right.\right.\right.$ $\left.\sigma(y)]) Q_{x, y}+Q_{x, V^{*}} / K \forall_{x \neq y, y \neq V^{*}}\right\}$,

- $\mathcal{H}_{b}=\left\{\hat{\sigma}\left(V^{*}\right)=b, \hat{p}_{\sigma(x), \sigma(y)}=\left(\left|\mathcal{V}_{\sigma(y)}\right|-\mathbb{1}[\sigma(y)=b]-\mathbb{1}\left[x \neq V^{*}, \sigma(x)=\right.\right.\right.$ $\left.\sigma(y)]) Q_{x, y}+Q_{x, V^{*}} / K \forall_{x \neq y, y \neq V^{*}}\right\}$.

Recall that because the algorithm is $(\varepsilon, 1)$-locally good at $(\alpha, p)$, it is able to cluster well in both (slightly different) BMCs in hypotheses $\mathcal{H}_{a}$ and $\mathcal{H}_{b}$. Note furthermore that both of these BMCs give the exact same $Q$-matrix, regardless of whether truly $\sigma\left(V^{*}\right)=a$ or $b$. Therefore, since $\mathcal{H}_{a}$ held w.p. $\alpha_{a} /\left(\alpha_{a}+\alpha_{b}\right)$ and $\mathcal{H}_{b}$ otherwise, this $(\varepsilon, 1)$-locally good algorithm at $(\alpha, p)$ will misclassify $V^{*}$ with probability at least $\mathbb{P}_{\Psi}\left[V^{*} \in \mathcal{E}\right] \geq\left(\alpha_{a} \wedge \alpha_{b}\right) /\left(\alpha_{a}+\alpha_{b}\right) \triangleq$ $\delta>0$.

Proof of Proposition 4 (ii). Select a state $V^{*}$ uniformly at random from any two specific clusters $a, b \in\{1, \ldots, K\}, a \neq b$. We are going to bound

$$
\mathbb{P}_{\Psi}[L \leq f(n, T)]=\mathbb{P}_{\Psi}\left[L \leq f(n, T), V^{*} \in \mathcal{E}\right]+\mathbb{P}_{\Psi}\left[L \leq f(n, T), V^{*} \notin \mathcal{E}\right] .
$$

for any function $f: \mathbb{N}_{+}^{2} \rightarrow \mathbb{R}$.

The first term of (71) can be bounded using our change of measure formula (20). Namely,

$$
\begin{aligned}
\mathbb{P}_{\Psi}\left[L \leq f(n, T), V^{*} \in \mathcal{E}\right] & \stackrel{(20)}{\leq} \mathrm{e}^{f(n, T)} \mathbb{P}_{\Phi}\left[L \leq f(n, T), V^{*} \in \mathcal{E}\right] \\
& \leq \mathrm{e}^{f(n, T)} \mathbb{P}_{\Phi}\left[V^{*} \in \mathcal{E}\right] .
\end{aligned}
$$

Because $V^{*}$ is selected from $\mathcal{V}_{a} \cup \mathcal{V}_{b}$ uniformly at random, we have by Lemma 16, see §SM6.1, that for any $V$ selected uniformly at random from all vertices $\mathcal{V}$,

$$
\mathbb{P}_{\Phi}\left[V^{*} \in \mathcal{E}\right]=\mathbb{P}_{\Phi}\left[V \in \mathcal{E} \mid V \in \mathcal{V}_{a} \cup \mathcal{V}_{b}\right]=\frac{\mathbb{P}_{\Phi}\left[V \in \mathcal{E}, V \in \mathcal{V}_{a} \cup \mathcal{V}_{b}\right]}{\mathbb{P}_{\Phi}\left[V \in \mathcal{V}_{a} \cup \mathcal{V}_{b}\right]} \leq \frac{\mathbb{P}_{\Phi}[V \in \mathcal{E}]}{\alpha_{a}+\alpha_{b}}
$$

Subsequently by Lemma 17, see §SM6.1,

$$
\mathbb{P}_{\Phi}\left[V^{*} \in \mathcal{E}\right] \leq \frac{\mathbb{E}_{\Phi}[|\mathcal{E}|]}{\left(\alpha_{a}+\alpha_{b}\right) n}
$$

imsart-aos ver. 2014/10/16 file: JS_AP_SY_CBMC__arXiv.tex date: July 31, 2019 
Substituting (74) into (72), we obtain

$$
\mathbb{P}_{\Psi}\left[L \leq f(n, T), V^{*} \in \mathcal{E}\right] \leq \mathrm{e}^{f(n, T)} \frac{\mathbb{E}_{\Phi}[|\mathcal{E}|]}{\left(\alpha_{a}+\alpha_{b}\right) n} .
$$

The second term of (71) can be bounded using Proposition 4 (i):

(76) $\mathbb{P}_{\Psi}\left[L \leq f(n, T), V^{*} \notin \mathcal{E}\right] \leq \mathbb{P}_{\Psi}\left[V^{*} \notin \mathcal{E}\right]=1-\mathbb{P}_{\Psi}\left[V^{*} \in \mathcal{E}\right] \leq 1-\delta<1$.

Now using (75) and (76) to bound (71), we arrive at

$$
\mathbb{P}_{\Psi}[L \leq f(n, T)] \leq \mathrm{e}^{f(n, T)} \frac{\mathbb{E}_{\Phi}[|\mathcal{E}|]}{\left(\alpha_{a}+\alpha_{b}\right) n}+1-\delta .
$$

We now prepare for an application of Chebyshev's inequality. First note using (77) that

$$
\mathbb{P}_{\Psi}[L \geq f(n, T)]=1-\mathbb{P}_{\Psi}[L \leq f(n, T)] \geq \delta-\mathrm{e}^{f(n, T)} \frac{\mathbb{E}_{\Phi}[|\mathcal{E}|]}{\left(\alpha_{a}+\alpha_{b}\right) n} .
$$

Specify $f(n, T)=\ln (\delta / 2)+\ln \left(\left(\alpha_{a}+\alpha_{b}\right) n / \mathbb{E}_{\Phi}[|\mathcal{E}|]\right)$, so that

$$
\mathbb{P}_{\Psi}\left[L \geq \ln \frac{\delta}{2}+\ln \frac{\left(\alpha_{a}+\alpha_{b}\right) n}{\mathbb{E}_{\Phi}[|\mathcal{E}|]}\right] \geq \frac{\delta}{2}
$$

Since $\delta>0$, we can apply Chebyshev's inequality and (78) to conclude

(80) $\mathbb{P}_{\Psi}\left[L \geq \mathbb{E}_{\Psi}[L]+\sqrt{\frac{2}{\delta}} \sqrt{\operatorname{Var}_{\Psi}[L]}\right] \leq \frac{\delta}{2} \leq \mathbb{P}_{\Psi}\left[L \geq \ln \frac{\delta}{2}+\ln \frac{\left(\alpha_{a}+\alpha_{b}\right) n}{\mathbb{E}_{\Phi}[|\mathcal{E}|]}\right]$

Comparing the events in the l.h.s. and r.h.s. of the above inequalities, we then must have

$$
\ln \frac{\delta}{2}+\ln \frac{\left(\alpha_{a}+\alpha_{b}\right) n}{\mathbb{E}_{\Phi}[|\mathcal{E}|]} \leq \mathbb{E}_{\Psi}[L]+\sqrt{\frac{2}{\delta}} \sqrt{\operatorname{Var}_{\Psi}[L]}
$$

Rearranging gives (30) with $C=\left(\alpha_{a}+\alpha_{b}\right) \delta / 2>0$. This completes the proof.

SM3.5. Proof of Proposition 5. Define $R_{x, y} \triangleq \ln \left(Q_{x, y} / P_{x, y}\right)$ for notational convenience: we refer to §SM6.2 for its asymptotic behavior. Since the Markov chain is started at equilibrium,

$$
\mathbb{E}_{\Psi}\left[L \mid \sigma\left(V^{*}\right)\right]=T \sum_{x \in \mathcal{V}} \sum_{y \in \mathcal{V}} \Pi_{x}^{(Q)} Q_{x, y} \ln R_{x, y}
$$

imsart-aos ver. 2014/10/16 file: JS_AP_SY__CBMC_-arXiv.tex date: July 31, 2019 
The largest individual contributions to the expectation in (82) are by jumps to and from $V^{*}$, since this is where the change of measure is modified most. Jumps not involving $V^{*}$ contribute less individually, but there are many of such jumps. We therefore separate out the jumps to and from $V^{*}$, i.e.,

$$
\begin{aligned}
\frac{\mathbb{E}_{\Psi}\left[L \mid \sigma\left(V^{*}\right)\right]}{T}= & \sum_{y \neq V^{*}} \Pi_{V^{*}}^{(Q)} Q_{V^{*}, y} \ln R_{V^{*}, y}+\sum_{x \neq V^{*}} \Pi_{x}^{(Q)} Q_{x, V^{*}} \ln R_{x, V^{*}} \\
& +\sum_{x, y \neq V^{*}} \Pi_{x}^{(Q)} Q_{x, y} \ln R_{x, y} .
\end{aligned}
$$

We now calculate the leading order behavior of each term.

For the first term in (83) we have by (i) Lemma 18 in $§ S M 6.2$ and $Q$ 's definition, see (25), and (ii) Proposition 3,

$$
\begin{aligned}
\sum_{y \neq V^{*}} \Pi_{V^{*}}^{(Q)} Q_{V^{*}, y} \ln R_{V^{*}, y} & \stackrel{(\mathrm{i})}{\sim} \sum_{k=1}^{K} \sum_{y \in \mathcal{W}_{k}} \Pi_{V^{*}}^{(Q)} \frac{q_{0, k}}{\left|\mathcal{W}_{k}\right|} \ln \frac{q_{0, \omega(y)}}{p_{\sigma\left(V^{*}\right), \omega(y)}} \\
& \stackrel{(\mathrm{ii})}{\sim} \frac{1}{n} \sum_{k=1}^{K} \gamma_{0}^{[1]} q_{0, k} \ln \frac{q_{0, k}}{p_{\sigma\left(V^{*}\right), k}} .
\end{aligned}
$$

The second term in (83) handles similarly:

$$
\begin{aligned}
\sum_{x \neq V^{*}} \Pi_{x}^{(Q)} Q_{x, V^{*}} \ln R_{x, V^{*}} & \stackrel{(\mathrm{i})}{\sim} \sum_{k=1}^{K} \sum_{x \in \mathcal{W}_{k} \backslash\left\{V^{*}\right\}} \bar{\Pi}_{k}^{(Q)} \frac{q_{k, 0}}{n} \ln \frac{q_{k, 0} \alpha_{\sigma\left(V^{*}\right)}}{p_{k, \sigma\left(V^{*}\right)}} \\
& \stackrel{(\mathrm{ii})}{\sim} \frac{1}{n} \sum_{k=1}^{K} \gamma_{k}^{[0]} q_{k, 0} \ln \frac{q_{k, 0} \alpha_{\sigma\left(V^{*}\right)}}{p_{k, \sigma\left(V^{*}\right)}}
\end{aligned}
$$

The third term in (83) requires (iii) a Taylor expansion of $\ln (1+x)=$ $x+O\left(x^{2}\right)$ for $x \approx 0$ and (iv) the balance equations (69)-(70), so that

$$
\begin{aligned}
& \sum_{x, y \neq V^{*}} \Pi_{x}^{(Q)} Q_{x, y} \ln R_{x, y} \stackrel{(\mathrm{iii})}{\sim} \sum_{k, l \neq 0} \sum_{x \in \mathcal{W}_{k}} \sum_{y \in \mathcal{W}_{l} \backslash\{x\}} \bar{\Pi}_{k}^{(Q)} \frac{q_{k, l}}{\left|\mathcal{W}_{l}\right|-\mathbb{1}[k=l]} \times \\
& \cdots \times \frac{1}{n}\left(\frac{\mathbb{1}\left[l=\sigma\left(V^{*}\right)\right]}{\alpha_{l}}-\frac{q_{k, 0}}{p_{k, l} K}\right) \stackrel{(24)}{\sim} \frac{1}{n} \sum_{k=1}^{K} \gamma_{k}^{[0]}\left(\frac{q_{k, \sigma\left(V^{*}\right)}}{\alpha_{\sigma\left(V^{*}\right)}}-\sum_{l=1}^{K} \frac{1}{K} q_{k, 0}\right) \\
& \stackrel{\text { iv) }}{=} \frac{1}{n}\left(\frac{\gamma_{\sigma\left(V^{*}\right)}^{[0]}}{\alpha_{\sigma\left(V^{*}\right)}}-\gamma_{0}^{[1]}\right) \text {. }
\end{aligned}
$$

Substituting (84)-(86) into (83) gives

$$
\mathbb{E}_{\Psi}\left[L \mid \sigma\left(V^{*}\right)\right] \sim \frac{T}{n} \sum_{k=1}^{K}\left(\gamma_{0}^{[1]} q_{0, k} \ln \frac{q_{0, k}}{p_{\sigma\left(V^{*}\right), k}}+\gamma_{k}^{[0]} q_{k, 0} \ln \frac{q_{k, 0} \alpha_{\sigma\left(V^{*}\right)}}{p_{k, \sigma\left(V^{*}\right)}}\right)
$$

imsart-aos ver. 2014/10/16 file: JS_AP_SY__CBMC__arXiv.tex date: July 31, 2019 


$$
+\frac{T}{n}\left(\frac{\gamma_{\sigma\left(V^{*}\right)}^{[0]}}{\alpha_{\sigma\left(V^{*}\right)}}-\gamma_{0}^{[1]}\right)
$$

By now applying Proposition 3, we complete the proof.

SM3.6. Proof of Proposition 6. Define $L_{t} \triangleq \ln \left(Q_{X_{t-1}, X_{t}} / P_{X_{t-1}, X_{t}}\right)$. Expanding, we obtain

$$
\operatorname{Var}_{\Psi}\left[L \mid \sigma\left(V^{*}\right)\right]=\operatorname{Var}_{\Psi}\left[\sum_{t=1}^{T} L_{t} \mid \sigma\left(V^{*}\right)\right]=\sum_{t=1}^{T} \sum_{s=1}^{T} \operatorname{Cov}_{\Psi}\left[L_{t}, L_{s} \mid \sigma\left(V^{*}\right)\right] .
$$

We now consider the cases $|t-s| \geq 2$ and $|t-s| \leq 1$, in that order. Since there are only $O(T)$ terms corresponding to $|t-s| \leq 1$ that contribute to the sum, we only provide crude bounds on these terms. On the contrary, there are as many as $O\left(T^{2}\right)$ terms corresponding to $|t-s| \geq 2$, we will need sharper bounds for these terms. As we will show for the cases where $|t-s| \geq 2$, we can derive a sharper bound when $|t-s| \gg t_{\text {mix }}(\varepsilon)$ because Proposition 2 implies that the covariances decay quickly.

First note that since (i) the process is started from equilibrium, we have for any $t, s \in\{1, \ldots, T\}$ that

$$
\begin{aligned}
\operatorname{Cov}_{\Psi}\left[L_{t}, L_{s} \mid \sigma\left(V^{*}\right)\right] & =\mathbb{E}_{\Psi}\left[L_{t} L_{s} \mid \sigma\left(V^{*}\right)\right]-\mathbb{E}_{\Psi}\left[L_{t} \mid \sigma\left(V^{*}\right)\right] \mathbb{E}_{\Psi}\left[L_{s} \mid \sigma\left(V^{*}\right)\right] \\
& \stackrel{(\mathrm{i})}{=} \mathbb{E}_{\Psi}\left[L_{t} L_{s} \mid \sigma\left(V^{*}\right)\right]-\mathbb{E}_{\Psi}\left[L_{t} \mid \sigma\left(V^{*}\right)\right]^{2}
\end{aligned}
$$

Consider the case $|t-s| \geq 2$. Define $S_{x, y, u, v} \triangleq\left(\ln R_{x, y}\right)\left(\ln R_{u, v}\right)$ for notational convenience. Refer to §SM6.2 for its asymptotic behavior. In this case the first term of (89) evaluates as

$$
\begin{aligned}
& \mathbb{E}_{\Psi}\left[L_{t} L_{s} \mid \sigma\left(V^{*}\right)\right] \\
= & \sum_{x, y, u, v} \mathbb{P}_{\Psi}\left[X_{t \wedge s-1}=x, X_{t \wedge s}=y, X_{t \vee s-1}=u, X_{t \vee s}=v \mid \sigma\left(V^{*}\right)\right] S_{x, y, u, v} \\
= & \sum_{x, y, u, v} \Pi_{x}^{(Q)} Q_{x, y}\left(\sum_{z_{t \wedge s+1}, \ldots, z_{t \vee s-2}} Q_{y, z_{t \wedge s+1}} Q_{z_{t \wedge s+2}, z_{t \wedge s+2}} \cdots Q_{z_{t \vee s-2}, u}\right) Q_{u, v} S_{x, y, u, v}
\end{aligned}
$$

$=\sum_{x, y, u, v} \Pi_{x}^{(Q)} Q_{x, y} Q_{y, u}^{|t-s|-1} Q_{u, v} S_{x, y, u, v}$.

The second term of (89) expands as

$$
\begin{aligned}
& \mathbb{E}_{\Psi}\left[L_{t} \mid \sigma\left(V^{*}\right)\right]^{2}=\left(\sum_{x, y} \Pi_{x}^{(Q)} Q_{x, y} \ln \frac{Q_{x, y}}{P_{x, y}}\right)^{2}=\sum_{x, y, u, v} \Pi_{x}^{(Q)} Q_{x, y} \Pi_{u}^{(Q)} Q_{u, v} S_{x, y, u, v} . \\
& \text { imsart-aos ver. } 2014 / 10 / 16 \text { file: JS_AP_SY_CBMC_-arXiv.tex date: July 31, } 2019
\end{aligned}
$$


Substituting (90) and (92) into the last member of (89) gives

$$
\operatorname{Cov}_{\Psi}\left[L_{t}, L_{s} \mid \sigma\left(V^{*}\right)\right]=\sum_{x, y, u, v} \Pi_{x}^{(Q)} Q_{x, y}\left(Q_{y, u}^{|t-s|-1}-\Pi_{u}^{(Q)}\right) Q_{u, v} S_{x, y, u, v}
$$

In order to bound (93), we need to take two effects into consideration: a filter effect that happens because the transition matrix $Q$ is similar to the transition matrix $P$, and a concentration effect because the Markov chain moves closer to equilibrium as time progresses. The filter effect is quantified by Corollary 2 in $\S$ SM6.2. The latter implies that $\sum_{x, y, u, v} S_{x, y, u, v} \leq c_{1} n^{2}$ for some absolute constant $c_{1}$ (even though $\sum_{x, y, u, v} 1=n^{4}$ ). We can use the effect by for example bounding $\Pi_{x}^{(Q)} Q_{x, y}\left(Q_{y, u}^{m}-\Pi_{u}^{(Q)}\right) Q_{u, v} \leq c_{2} / n^{4}$ uniformly using another absolute constant, and then concluding that $\operatorname{Cov}_{\Psi}\left[L_{t}, L_{s} \mid \sigma\left(V^{*}\right)\right] \leq$ $c_{2}\left(T^{2} / n^{4}\right) \sum_{x, y, u, v} S_{x, y, u, v} \leq c_{1} c_{2} T^{2} / n^{2}$. However, this bound is not sufficiently sharp for our purposes: we need to provide a bound that is at least $o\left(T^{2} / n^{2}\right)$.

To arrive at a sharper bound, we use the concentration of the Markov chain. Apply the triangle inequality first, and then bound $\Pi_{x}^{(Q)} Q_{x, y} Q_{u, v} \leq c_{1} / n^{3}$ uniformly using an absolute constant $c_{1}$ to obtain

$$
\begin{aligned}
& \left|\sum_{t=1}^{T} \sum_{s=1}^{T} \mathbb{1}[|t-s| \geq 2] \operatorname{Cov}_{\Psi}\left[L_{t}, L_{s} \mid \sigma\left(V^{*}\right)\right]\right| \\
& \leq \frac{2 c_{1}}{n^{3}} \sum_{t=1}^{T} \sum_{s=t+2}^{T} \sum_{x, y, u, v}\left|Q_{y, u}^{|t-s|-1}-\Pi_{u}^{(Q)}\right|\left|S_{x, y, u, v}\right| .
\end{aligned}
$$

Now let $m \in \mathbb{N}_{+}$. By nonnegativity of the summands and (6), $\left|Q_{x, y}^{m}-\Pi_{y}^{(Q)}\right| \leq$ $\sum_{y}\left|Q_{x, y}^{m}-\Pi_{y}^{(Q)}\right|=2 d_{\mathrm{TV}}\left(Q_{x, \cdot}^{m}, \Pi^{(Q)}\right)$. Recall furthermore from (63) and (64) combined that there exists a $\delta(Q) \in(0,1)$ such that $d_{\mathrm{TV}}\left(Q_{u, \cdot}^{m}, \Pi\right) \leq$ $(\delta(Q))^{m} d_{\mathrm{TV}}\left(Q_{u, \cdot}^{0}, \Pi\right)$ for $u \in \mathcal{V}$. We therefore have that there exists an absolute constant $c_{3}$ such that

$$
\begin{aligned}
& \left|\sum_{t=1}^{T} \sum_{s=1}^{T} \mathbb{1}[|t-s| \geq 2] \operatorname{Cov}_{\Psi}\left[L_{t}, L_{s} \mid \sigma\left(V^{*}\right)\right]\right| \\
& \leq \frac{4 c_{1} c_{2} \max _{u \in \mathcal{V}}\left\{d_{\mathrm{TV}}\left(Q_{u, \cdot}^{0}, \Pi\right)\right\}}{n^{3}} \sum_{t=1}^{T} \sum_{s=t+2}^{T}(\delta(Q))^{|t-s|-1} \sum_{x, y, u, v}\left|S_{x, y, u, v}\right| \\
& \stackrel{\text { (i) }}{\leq} \frac{c_{3}}{n} \sum_{t=1}^{T} \sum_{s=t+2}^{T}(\delta(Q))^{|t-s|-1},
\end{aligned}
$$

imsart-aos ver. 2014/10/16 file: JS_AP_SY__CBMC__arXiv.tex date: July 31, 2019 
due to (i) the filter effect. By a continuous extension of the sum,

$$
\sum_{t=1}^{T} \sum_{s=t+2}^{T}(\delta(Q))^{|t-s|-1} \leq \int_{0}^{T} \int_{t+1}^{T}(\delta(Q))^{|t-s|-1} \mathrm{~d} s \mathrm{~d} t \sim-\frac{T}{\ln (\delta(Q))} .
$$

Since $\delta(Q) \in(0,1)$, there thus exists an absolute constant $c_{4}>0$ such that

$$
\left|\sum_{t=1}^{T} \sum_{s=1}^{T} \mathbb{1}[|t-s| \geq 2] \operatorname{Cov}_{\Psi}\left[L_{t}, L_{s} \mid \sigma\left(V^{*}\right)\right]\right| \leq c_{4} \frac{T}{n} .
$$

Finally we deal with the cases $|t-s| \leq 1$. When $|t-s|=0$, or equivalently $t=s$, we have that (iv) because of Lemma 18 and Corollary 2 that there exist absolute constants $c_{5}, \ldots, c_{8}$ such that

$$
\begin{aligned}
& \operatorname{Cov}_{\Psi}\left[L_{t}, L_{t} \mid \sigma\left(V^{*}\right)\right] \stackrel{(89)}{\leq} \mathbb{E}_{\Psi}\left[L_{t}^{2} \mid \sigma\left(V^{*}\right)\right]=\sum_{x \in \mathcal{V}} \sum_{y \in \mathcal{V}} \Pi_{x}^{(Q)} Q_{x, y}\left(\ln R_{x, y}\right)^{2} \\
& \stackrel{\text { (iv) }}{\leq} \Pi_{V^{*}}^{(Q)} \sum_{y \neq V^{*}} Q_{V^{*}, y} c_{5}+\sum_{x \neq V^{*}} \Pi_{x}^{(Q)} Q_{x, V^{*}} c_{6}+\sum_{x \neq V^{*}} \sum_{y \neq V^{*}} \Pi_{x}^{(Q)} Q_{x, y} \frac{c_{7}}{n^{2}} \leq \frac{c_{8}}{n}
\end{aligned}
$$

for all $t=1, \ldots, T$. Therefore

$$
\left|\sum_{t=1}^{T} \sum_{s=1}^{T} \mathbb{1}[|t-s|=0] \operatorname{Cov}_{\Psi}\left[L_{t}, L_{s} \mid \sigma\left(V^{*}\right)\right]\right|=\sum_{t=1}^{T} \operatorname{Var}_{\Psi}\left[L_{t} \mid \sigma\left(V^{*}\right)\right]=O\left(\frac{T}{n}\right) .
$$

When $|t-s|=1$, there exists an absolute constant $c_{9}>0$ such that

$$
\begin{aligned}
& \operatorname{Cov}_{\Psi}\left[L_{t \wedge s}, L_{t \wedge s+1} \mid \sigma\left(V^{*}\right)\right] \stackrel{(89)}{\leq} \mathbb{E}_{\Psi}\left[L_{t \wedge s} L_{t \wedge s+1} \mid \sigma\left(V^{*}\right)\right] \\
& \leq \sum_{x, y, z} \Pi_{x}^{(Q)} Q_{x, y} Q_{y, z} S_{x, y, y, z} \leq \frac{c_{9}}{n^{3}} \sum_{x, y, z} S_{x, y, y, z} .
\end{aligned}
$$

Invoking Corollary 2's filter effect implies that $\sum_{x, y, z} S_{x, y, y, z}=O\left(n^{2}\right)$. Therefore

$$
\left|\sum_{s, t=1}^{T} \mathbb{1}[|t-s|=1] \operatorname{Cov}_{\Psi}\left[L_{t}, L_{s} \mid \sigma\left(V^{*}\right)\right]\right| \leq 2 \sum_{t=1}^{T}\left|\operatorname{Cov}_{\Psi}\left[L_{t}, L_{t+1} \mid \sigma\left(V^{*}\right)\right]\right|=O\left(\frac{T}{n}\right) .
$$

Splitting (88) into the respective cases and then (v) substituting (97), (99), and (101) gives

$$
\operatorname{Var}_{\Psi}\left[L \mid \sigma\left(V^{*}\right)\right]=\sum_{t=1}^{T} \sum_{s=1}^{T}(\mathbb{1}[t=s]+\mathbb{1}[|t-s|=1]
$$




$$
+\mathbb{1}[|t-s| \geq 2]) \operatorname{Cov}_{\Psi}\left[L_{t}, L_{s} \mid \sigma\left(V^{*}\right)\right] \stackrel{(\mathrm{v})}{=} O\left(\frac{T}{n}\right),
$$

which completes the proof.

SM3.7. Proof of Corollary 1. Let $a \neq b$ be any two distinct clusters, and let $q \in Q(a, b)$ (i.e., such that $I_{a}(q \| p)=I_{b}(q \| p)$. Select $V^{*}$ uniformly at random in $\mathcal{V}_{a} \cup \mathcal{V}_{b}$. Then we have:

$$
\begin{aligned}
\mathbb{E}_{\Psi}[L] & =\frac{\alpha_{a}}{\alpha_{a}+\alpha_{b}} \mathbb{E}_{\Psi}\left[L \mid \sigma\left(V^{*}\right)=a\right]+\frac{\alpha_{b}}{\alpha_{a}+\alpha_{b}} \mathbb{E}_{\Psi}\left[L \mid \sigma\left(V^{*}\right)=b\right] \\
& =\frac{T}{n} I_{a, b}(p)+o\left(\frac{T}{n}\right),
\end{aligned}
$$

where the last equality results from Proposition 5. Moreover, since for any random variables $X$ and $Y, \operatorname{Var}[X]=\mathbb{E}_{Y}[\operatorname{Var}[X \mid Y]]+\operatorname{Var}_{Y}[\mathbb{E}[X \mid Y]]$, we also have:

$\operatorname{Var}_{\Psi}[L]=\sum_{k \in\{a, b\}} \frac{\alpha_{k}}{\alpha_{a}+\alpha_{b}}\left(\operatorname{Var}_{\Psi}\left[L \mid \sigma\left(V^{*}\right)=k\right]+\left(\mathbb{E}_{\Psi}\left[L \mid \sigma\left(V^{*}\right)=k\right]-\mathbb{E}_{\Psi}[L]\right)^{2}\right)$.

We deduce that $\operatorname{Var}_{\Psi}[L]=o\left(T^{2} / n^{2}\right)$ from Proposition 6 for the first two terms, and from the fact that $q \in Q(a, b)$ for the last two terms.

SM3.8. Proof of Lemma 3. Recall the definition of $q_{c}$ for $c=1, \ldots, K$ :

$$
q_{c}=\left(0, \frac{p_{1, c}}{\alpha_{c}}, \ldots, \frac{p_{K, c}}{\alpha_{c}} ; 0, p_{c, 1}, \ldots, p_{c, K}\right) \in \mathcal{Q} .
$$

Let $a^{*}$ and $b^{*}$ be the cluster indices such that

$$
I(\alpha, p)=\sum_{k=1}^{K} \frac{1}{\alpha_{a^{*}}}\left(\pi_{a^{*}} p_{a^{*}, k} \ln \frac{p_{a^{*}, k}}{p_{b^{*}, k}}+\pi_{k} p_{k, a^{*}} \ln \frac{p_{k, a^{*}}}{p_{k, b^{*}}}+\left(\frac{\pi_{b^{*}}}{\alpha_{b^{*}}}-\frac{\pi_{a^{*}}}{\alpha_{a^{*}}}\right)\right) .
$$

From the definitions of $I(\alpha, p), I_{c}(q \| p)$, and $q_{c}$, we have that (i) $I_{a^{*}}\left(q_{a^{*}} \| p\right)=0$, $I_{a^{*}}\left(q_{b^{*}} \| p\right)=I(\alpha, p)$, and (ii) $I_{b^{*}}\left(q_{b^{*}} \| p\right)=0, I_{b^{*}}\left(q_{a^{*}} \| p\right) \geq I(\alpha, p)$.

We are now going to show that there exists a path along which $I_{a^{*}}(q \| p)$ monotonically decreases from $I(\alpha, p)$ to 0 , while at the same time the $I_{b^{*}}(q \| p)$ moves from initially 0 to eventually $I_{b^{*}}\left(q_{a^{*}} \| p\right) \geq I(\alpha, p)$. Since $I_{c}(q \| p)$ is continuous in $q$, this implies the existence of atleast one point $\bar{q}$ such that $0 \leq I_{a^{*}}(\bar{q} \| p)=I_{b^{*}}(\bar{q} \| p) \leq I(\alpha, p)$.

First, we will walk along the path

$$
q^{(1)}(\lambda)=(1-\lambda)\left(\frac{p_{1, b^{*}}}{\alpha_{b^{*}}}, \ldots, \frac{p_{K, b^{*}}}{\alpha_{b^{*}}} ; p_{b^{*}, 1}, \ldots, p_{b^{*}, K} ; 0\right)
$$

imsart-aos ver. 2014/10/16 file: JS_AP_SY__CBMC_-arXiv.tex date: July 31, 2019 


$$
+\lambda\left(\frac{p_{1, b^{*}}}{\alpha_{b^{*}}}, \ldots, \frac{p_{K, b^{*}}}{\alpha_{b^{*}}} ; p_{a^{*}, 1}, \ldots, p_{a^{*}, K} ; 0\right) .
$$

parameterized by $\lambda \in[0,1]$. Specifically note that $I_{a^{*}}\left(q^{(1)}(\lambda) \| p\right)$ is convex and monotonically decreasing in $\lambda$. This is because $\lambda$ only changes the convex summands $\left(\sum_{k=1}^{K} \pi_{l} q_{l, 0}\right) \operatorname{KL}\left(q_{0,} \cdot \| p_{c, .}\right)$ in $(29)$, and additionally, $\operatorname{KL}\left(q_{0, \cdot} . \| p_{c, .}\right)$ is minimized at $\lambda=1$.

Next, starting from the end point $q^{(1)}(1)$, we will walk along the path

$$
\begin{aligned}
q^{(2)}(\eta)= & (1-\eta)\left(\frac{p_{1, b^{*}}}{\alpha_{b^{*}}}, \ldots, \frac{p_{K, b^{*}}}{\alpha_{b^{*}}} ; p_{a^{*}, 1}, \ldots, p_{a^{*}, K} ; 0\right) \\
& +\eta\left(\frac{p_{1, a^{*}}}{\alpha_{a^{*}}}, \ldots, \frac{p_{K, a^{*}}}{\alpha_{a^{*}}} ; p_{a^{*}, 1}, \ldots, p_{a^{*}, K} ; 0\right)
\end{aligned}
$$

parameterized by $\eta \in[0,1]$. Similar to before $I_{a^{*}}\left(q^{(2)}(\lambda) \| p\right)$ is convex and monotonically decreasing in $\eta$, and tends to 0 as $\eta \rightarrow 1$. Note that we have that $I_{b^{*}}\left(q^{(1)}(0) \| p\right)=0$ and $I_{b^{*}}\left(q^{(2)}(1) \| p\right)=I_{b^{*}}\left(q_{a^{*}} \| p\right) \geq I(\alpha, p)$.

SM3.9. Proof of Lemma 1. Recall that

$$
\mathbb{E}_{\Psi}\left[L \mid \sigma\left(V^{*}\right)\right]=\sum_{\text {all sample paths } \chi} \mathbb{P}_{\Psi}\left[\chi \mid \sigma\left(V^{*}\right)\right] \ln \frac{\mathbb{P}_{\Psi}\left[\chi \mid \sigma\left(V^{*}\right)\right]}{\mathbb{P}_{P}[\chi]}
$$

is a KL-divergence. As a consequence, $\mathbb{E}_{\Psi}[L]=0$ if and only if (107)

$$
\mathbb{P}_{\Psi}\left[\chi \mid \sigma\left(V^{*}\right)\right]=\prod_{t=1}^{T} Q_{x_{t-1}, x_{t}}=\prod_{t=1}^{T} P_{x_{t-1}, x_{t}}=\mathbb{P}_{P}[\chi] \quad \text { for all sample paths } \chi .
$$

Equivalently $\mathbb{E}_{\Psi}\left[L \mid \sigma\left(V^{*}\right)\right]=0$ if and only if $Q_{x, y}=P_{x, y}$ for all $x, y \in \mathcal{V}$, which can be seen by considering the set of paths that disagree only on the last jump. Since

$$
I_{\sigma\left(V^{*}\right)}(q \| p)=\lim _{n \rightarrow \infty} \frac{n}{T}\left(\mathbb{E}_{\Psi}\left[L \mid \sigma\left(V^{*}\right)\right]+o(1)\right),
$$

we obtain that $I_{\sigma\left(V^{*}\right)}(q \| p)=0$ if and only if $q=q_{\sigma\left(V^{*}\right)}$. Since there exists $\bar{q}$ such that $I_{a^{*}}(\bar{q} \| p)=I_{b^{*}}(\bar{q} \| p)=0, q_{a^{*}}=q_{b^{*}}$. This completes the proof.

\section{SM4. Proofs of Chapter 6.}

SM4.1. Proof of Proposition 7. Let $\operatorname{diag}(\Pi) \in[0,1]^{n \times n}$ denote the matrix whose diagonal entries correspond to the entries of $\Pi$. Then by (12), $N \triangleq$ $\mathbb{E}[\hat{N}]=T \operatorname{diag}(\Pi) P$. 
The Markov process $\left\{X_{t}\right\}_{0 \leq t \leq T}$ generates $\hat{N}$. We can think of $\hat{N}$ as a sum of random matrices $\hat{N}=\sum_{t=0}^{T-1} \hat{N}(t)$, where all the elements of each $t$-th matrix $\hat{N}(t) \in\{0,1\}^{n \times n}$ are zero except for the one element $(\hat{N}(t))_{X_{t}, X_{t+1}}=1$. It is important to note that matrices $\hat{N}(t)$ and $\hat{N}(t-1)$ are dependent. In particular, only the $X_{t}$-th row of $\hat{N}(t)$ can contain a nonzero value. These dependencies are what make the analysis challenging.

To circumvent the difficulties associated with these dependencies, we use the following trick. We split $\hat{N}$ into two parts, specifically,

$$
\hat{N}^{(\text {even })}=\sum_{t=0}^{\lceil T / 2\rceil-1} \hat{N}(2 t) \quad \text { and } \quad \hat{N}^{\text {(odd })}=\sum_{t=0}^{\lfloor T / 2\rfloor-1} \hat{N}(2 t+1) .
$$

This particular split ensures that the elements of $\hat{N}^{\text {(even) }}$ and $\hat{N}^{(\text {odd) }}$ are almost independent. Consider for instance the dependency between a matrix $\hat{N}(t)$ and the matrix $\hat{N}(t-2)$. Note that $\hat{N}(t)$ can contain a nonzero element almost anywhere when comparing to $\hat{N}(t-2)$, and that the only exceptions are diagonal entries.

Let us formalize this more precisely. Define $\bar{N}(t)=\mathbb{E}_{P}[\hat{N}(t) \mid \hat{N}(t-2)]$ for $t \geq 2$ and $\bar{N}(t)=\operatorname{diag}(\Pi) P$ for $t=1,2$. Note that $\mathbb{E}_{P}[\hat{N}(t) \mid \hat{N}(t-2)]=$ $\mathbb{E}_{P}[\hat{N}(t) \mid \hat{N}(t-2), \hat{N}(t-4), \ldots]$ for all $t \geq 2$ since $\left\{X_{t}\right\}_{0 \leq t \leq T}$ is a Markov chain. Hence, we have $\bar{N}(t)=\operatorname{diag}\left(P_{X_{t-1},}\right) P$ for $t \geq 2$. This trick of separating the original process into two processes each of which skips one unit of time ensures that almost all elements of $\bar{N}(t)$ are of order $1 / n^{2}$. Note that there exists an absolute constant $p_{\max }>0$ such that $\left(\operatorname{diag}\left(P_{x,}\right) P\right)_{y, z} \leq p_{\max } / n^{2}$ for all $x, y, z \in \mathcal{V}$.

We now explain how to show $\left\|\hat{N}_{\Gamma}-N\right\|=O_{\mathbb{P}}(\sqrt{(T / n) \ln (T / n)})$. Using the triangle inequality, it follows that $\left\|\hat{N}_{\Gamma}-N\right\| \leq\left\|\hat{N}_{\Gamma}-\sum_{t=0}^{T-1} \bar{N}(t)\right\|+$ $\left\|N-\sum_{t=0}^{T-1} \bar{N}(t)\right\|$. To prove the proposition, we will first show that

$$
\left\|N-\sum_{t=0}^{T-1} \bar{N}(t)\right\|=O_{\mathbb{P}}\left(\sqrt{\frac{T}{n} \ln \frac{T}{n}}\right)
$$

and then that

$$
\left\|\hat{N}_{\Gamma}-\sum_{t=0}^{T-1} \bar{N}(t)\right\|=O_{\mathbb{P}}\left(\sqrt{\frac{T}{n} \ln \frac{T}{n}}\right) .
$$

Part 1. Proof of (110). The Frobenius norm provides an upper bound of the spectral norm. Namely,

$$
\left\|N-\sum_{t=0}^{T-1} \bar{N}(t)\right\| \leq\left\|\left(\sum_{t=2}^{T-1}\left(\operatorname{diag}(\Pi)-\operatorname{diag}\left(P_{X_{t-1},}\right)\right)\right) P\right\|_{\mathrm{F}} .
$$

imsart-aos ver. 2014/10/16 file: JS_AP_SY__CBMC__arXiv.tex date: July 31, 2019 
Observe that for any matrix $A,\|\operatorname{diag}(A) P\|_{\mathrm{F}} \leq \max _{x \in \mathcal{V}}\left\{\left\|P_{x, .}\right\|_{2}\right\}\|A\|_{2}$ by the Cauchy-Schwarz inequality. Also note that $\left\|P_{x, \cdot}\right\|_{2}=O(1 / \sqrt{n})$ for all $x \in \mathcal{V}$. Hence to establish (110), it is sufficient to show that

$$
\left\|\sum_{t=2}^{T-1}\left(\Pi-P_{X_{t-1},}\right)\right\|_{2}=O_{\mathbb{P}}\left(\sqrt{T \ln \frac{T}{n}}\right) .
$$

In fact (113) can readily be verified. Centering, and using the triangle inequality, we have

$$
\begin{aligned}
\left\|\sum_{t=2}^{T-1}\left(\Pi-P_{X_{t-1},}\right)\right\|_{2} \leq & \left\|\sum_{t=2}^{T-1} \Pi-\sum_{i=1}^{K} \frac{\hat{N}_{\mathcal{V}, \mathcal{V}_{k}}}{\left|\mathcal{V}_{k}\right|} \sum_{v \in \mathcal{V}_{i}} P_{v}, \cdot\right\|_{2} \\
& +\left\|\sum_{i=1}^{K} \frac{\hat{N}_{\mathcal{V}, \mathcal{V}_{k}}}{\left|\mathcal{V}_{k}\right|} \sum_{x \in \mathcal{V}_{k}} P_{x, \cdot}-\sum_{t=2}^{T-1} P_{X_{t-1}, \cdot}\right\|_{2} .
\end{aligned}
$$

To bound the first term in the r.h.s. of (114), recall first that (i) $\Pi_{y}=$ $\sum_{x \in \mathcal{V}} \Pi_{x} P_{x, y}, N_{x, y}=T \Pi_{x} P_{x, y}, \forall_{x, y}$, and (ii) $\Pi_{x}=\bar{\Pi}_{\sigma(x)} \forall_{x}$. Therefore (iii) $T \bar{\Pi}_{k}\left|\mathcal{V}_{k}\right|=N_{\mathcal{V}, V_{k}}$. Thus

$$
\begin{aligned}
& \left\|\sum_{t=2}^{T-1} \Pi-\sum_{k=1}^{K} \frac{\hat{N}_{\mathcal{V}, \mathcal{V}_{k}}}{\left|\mathcal{V}_{k}\right|} \sum_{x \in \mathcal{V}_{k}} P_{x, \cdot}\right\|_{2}^{2} \stackrel{(\mathrm{i})}{=} \sum_{y \in \mathcal{V}}\left|(T-2) \Pi_{y}-\sum_{k=1}^{K} \frac{\hat{N}_{\mathcal{V}, \mathcal{V}_{i}}}{\left|\mathcal{V}_{i}\right|} \sum_{x \in \mathcal{V}_{k}} P_{x, y}\right|^{2} \\
& \stackrel{\text { (ii) }}{=} \sum_{y \in \mathcal{V}}\left|(T-2) \sum_{k=1}^{K} \sum_{x \in \mathcal{V}_{k}} \bar{\Pi}_{k} P_{x, y}-\sum_{k=1}^{K} \frac{\hat{N}_{\mathcal{V}, \mathcal{V}_{k}}}{\left|\mathcal{V}_{k}\right|} \sum_{x \in \mathcal{V}_{k}} P_{x, y}\right|^{2} \\
& \stackrel{\text { (iii) }}{=} \sum_{y \in \mathcal{V}}\left|\sum_{k=1}^{K} \frac{\sum_{x \in \mathcal{V}_{k}} P_{x, y}}{\left|\mathcal{V}_{k}\right|}\left[\frac{T-2}{T} N_{\mathcal{V}, \mathcal{V}_{k}}-\hat{N}_{\mathcal{V}, \mathcal{V}_{k}}\right]\right|^{2} \\
& =O\left(\frac{1}{n}\right)\left|\sum_{k=1}^{K}\left[\frac{T-2}{T} N_{\mathcal{V}, \mathcal{V}_{k}}-\hat{N}_{\mathcal{V}, \mathcal{V}_{k}}\right]\right|^{2}=O_{\mathbb{P}}\left(\frac{T}{n} \ln \frac{T}{n}\right)
\end{aligned}
$$

where the last equality follows from (39). To bound the second term in the r.h.s. of (114), note that (iv) $\hat{N}_{\mathcal{V}, x}=\sum_{y \in \mathcal{V}} \hat{N}_{y, x}=\sum_{y \in \mathcal{V}} \sum_{t=0}^{T-1} \mathbb{1}\left[X_{t}\right.$ $\left.=y, X_{t+1}=x\right]=\sum_{t=0}^{T-1} \mathbb{1}\left[X_{t+1}=x\right]$ by definition, and write

$$
\begin{aligned}
& \left\|\sum_{k=1}^{K} \hat{N}_{\mathcal{V}, \mathcal{V}_{k}} \frac{\sum_{x \in \mathcal{V}_{k}} P_{x, \cdot}}{\left|\mathcal{V}_{k}\right|}-\sum_{t=2}^{T-1} P_{X_{t-1} \cdot}\right\|_{2}^{2}=\sum_{y \in \mathcal{V}}\left|\sum_{k=1}^{K} \hat{N}_{\mathcal{V}, \mathcal{V}_{k}} \frac{\sum_{x \in \mathcal{V}_{k}} P_{x, y}}{\left|\mathcal{V}_{k}\right|}-\sum_{t=2}^{T-1} P_{X_{t-1}, y}\right|^{2} \\
& =\sum_{y \in \mathcal{V}}\left|\sum_{k=1}^{K} \hat{N}_{\mathcal{V}, \mathcal{V}_{k}} \frac{\sum_{x \in \mathcal{V}_{k}} P_{x, y}}{\left|\mathcal{V}_{k}\right|}-\sum_{x \in \mathcal{V}} \sum_{t=2}^{T-1} P_{x, y} \mathbb{1}\left[X_{t-1}=x\right]\right|^{2}
\end{aligned}
$$

imsart-aos ver. 2014/10/16 file: JS_AP_SY_CBMC__arXiv.tex date: July 31, 2019 


$$
\begin{aligned}
& \stackrel{\text { (iv) }}{=} \sum_{y \in \mathcal{V}}\left|\sum_{k=1}^{K}\left(\hat{N}_{\mathcal{V}, \mathcal{V}_{k}} \frac{\sum_{x \in \mathcal{V}_{k}} P_{x, y}}{\left|\mathcal{V}_{k}\right|}-\sum_{x \in \mathcal{V}_{k}} P_{x, y}\left(\hat{N}_{\mathcal{V}, x}-\mathbb{1}\left[X_{T-1}=x\right]-\mathbb{1}\left[X_{T}=x\right]\right)\right)\right|^{2} \\
& \leq 2 \sum_{y \in \mathcal{V}}\left|\sum_{k=1}^{K} \hat{N}_{\mathcal{V}, \mathcal{V}_{k}} \frac{\sum_{x \in \mathcal{V}_{k}} P_{x, y}}{\left|\mathcal{V}_{k}\right|}-\sum_{k=1}^{K} \sum_{x \in \mathcal{V}_{k}} P_{x, y} \hat{N}_{\mathcal{V}, x}\right|^{2} \\
& \quad+2 \sum_{y \in \mathcal{V}}\left|\sum_{k=1}^{K} \sum_{x \in \mathcal{V}_{k}} P_{x, y}\left(\mathbb{1}\left[X_{T-1}=x\right]+\mathbb{1}\left[X_{T}=x\right]\right)\right|^{2} \\
& \leq 2 \sum_{y \in \mathcal{V}}\left|\sum_{k=1}^{K} \max _{w, z \in \mathcal{V}_{k}}\right| \hat{N}_{\mathcal{V}, w}-\hat{N}_{\mathcal{V}, z}\left|\frac{\sum_{x \in \mathcal{V}_{k}} P_{x, y}}{\left|\mathcal{V}_{k}\right|}\right|^{2} \\
& \quad+2 \sum_{y \in \mathcal{V}}\left|\sum_{k=1}^{K} \sum_{x \in \mathcal{V}_{k}} P_{x, y}\left(\mathbb{1}\left[X_{T-1}=x\right]+\mathbb{1}\left[X_{T}=x\right]\right)\right|^{2} \\
& \stackrel{(\mathrm{v})}{=} 2 \sum_{y \in \mathcal{V}}\left|\sum_{k=1}^{K} \sum_{x \in \mathcal{V}_{k}} P_{x, y}\left(\mathbb{1}\left[X_{T-1}=x\right]+\mathbb{1}\left[X_{T}=x\right]\right)\right|^{2}+O_{\mathbb{P}}\left(\frac{T}{n^{2}} \ln n^{2}\right) \\
& (116) \\
& =O_{\mathbb{P}}\left(\frac{T}{n} \ln \frac{T}{n}\right),
\end{aligned}
$$

where (v) stems from (41). This proves (113) after taking the square root.

Part 2. Proof of (111). Using the triangle inequality, we obtain

$$
\left\|\hat{N}_{\Gamma}-\sum_{t=0}^{T-1} \bar{N}(t)\right\| \leq\left\|\sum_{t=0}^{\left\lceil\frac{T}{2}\right\rceil-1}\left(\hat{N}_{\Gamma}(2 t)-\bar{N}(2 t)\right)\right\|+\left\|\sum_{t=0}^{\left\lfloor\frac{T}{2}\right\rfloor-1}\left(\hat{N}_{\Gamma}(2 t+1)-\bar{N}(2 t+1)\right)\right\| .
$$

We next show that the first term in the r.h.s. of (117) is in fact $O_{\mathbb{P}}(\sqrt{T / n}$ $\ln (T / n))$. The second term can be bounded using an analogous argument. The proof classically consists in relating the spectral norm to the rectangular quotient, and then combining an $\varepsilon$-net argument with a sufficiently strong concentration inequality.

To simplify notation, define $A \triangleq \sum_{t=0}^{\lceil T / 2\rceil-1} \hat{N}(2 t), M \triangleq \sum_{t=0}^{\lceil T / 2\rceil-1} \bar{N}(2 t)$, and $f_{n}=\sqrt{(T / n) \ln (T / n)}$. We wish to bound $\left\|A_{\Gamma}-M\right\|$. To this aim, we use the rectangular quotient relation [33]:

$$
\left\|A_{\Gamma}-M\right\|=\sigma_{1}=\max _{x, y \in \mathbb{S}^{n-1}}\left|x^{\mathrm{T}}\left(A_{\Gamma}-M\right) y\right|
$$

where $\mathbb{S}^{n-1}$ denotes the unit sphere in $\mathbb{R}^{n}$. 
Step 1: The $\epsilon$-net argument. Let us first recall the definition of an $\epsilon$-net: let $(X, d)$ be a metric space and let $\epsilon>0$. A subset $\mathcal{N}_{\epsilon}$ of $X$ is called an $\epsilon$-net of $X$ if every point $x \in X$ can be approximated to within $\epsilon$ by some point $y \in \mathcal{N}_{\epsilon}$, i.e., so that $d(x, y) \leq \epsilon$. To bound $\left\|A_{\Gamma}-M\right\|$, we use the classical $\epsilon$-net argument formalized in Lemma 8.

Lemma 8. Let $\mathcal{N}_{\epsilon}$ denote an $\epsilon$-net of $\mathbb{S}^{n-1}$ for some $\epsilon \in[0,1)$. Then

$$
\left\|A_{\Gamma}-M\right\| \leq \frac{1}{1-3 \epsilon} \max _{x, y \in \mathcal{N}_{\epsilon}}\left|x^{\mathrm{T}}\left(A_{\Gamma}-M\right) y\right| .
$$

Proof. We use here the notation $B=A_{\Gamma}-M$. Adapting the strategy in [34, Lemma 5.4]: Choose $a, b \in \mathbb{S}^{n-1}$ such that $\|B\|=\left|a^{\mathrm{T}} B b\right|$ and choose $x, y \in \mathcal{N}_{\epsilon}$ such that $\|x-a\|_{2} \leq \epsilon$ and $\|y-b\|_{2} \leq \epsilon$. Then by the triangle inequality,

$$
\left|x^{\mathrm{T}} B y-a^{\mathrm{T}} B b\right| \leq\left|(x-a)^{\mathrm{T}} B(y-b)\right|+\left|a^{\mathrm{T}} B(y-b)\right|+\left|(x-a)^{\mathrm{T}} B b\right| \leq
$$

$\|x-a\|_{2}\|B\|\|y-b\|_{2}+\|a\|_{2}\|B\|\|y-b\|_{2}+\|x-a\|_{2}\|B\|\|b\|_{2}=\left(2 \epsilon+\epsilon^{2}\right)\|B\|$.

Therefore $\left|x^{\mathrm{T}} B y\right| \geq\left(1-2 \epsilon-\epsilon^{2}\right)\|B\|$. By first maximizing over such $x, y$ and next extending the optimization range, we obtain

$$
\left(1-2 \epsilon-\epsilon^{2}\right)\|B\| \leq \max _{\left\{x, y \in \mathcal{N}_{\epsilon}:\|x-a\|_{2} \leq \epsilon,\|y-a\|_{2} \leq \epsilon\right\}}\left|x^{\mathrm{T}} B y\right| \leq \max _{x, y \in \mathcal{N}_{\epsilon}}\left|x^{\mathrm{T}} B y\right|
$$

That completes the proof of Lemma 8 .

By a volume covering argument there exists an $\epsilon$-net of $\mathbb{S}^{n-1}$ satisfying $\left|\mathcal{N}_{\epsilon}\right| \leq \mathrm{e}^{\zeta_{\epsilon} n}$ with $\zeta_{\epsilon}=\ln (1+2 / \epsilon)[34$, Lemma 5.2]. In the remainder of this proof, we use such an $\epsilon$-net.

Step 2: Splitting between light and heavy couples. To bound $\max _{x, y \in \mathcal{N}_{\epsilon}} \mid x^{\mathrm{T}}\left(A_{\Gamma}\right.$ $-M) y \mid$, we adapt the proof strategy used in [31]. Let us fix $x, y \in \mathcal{N}_{\epsilon}$. Define $\mathcal{L}=\left\{(v, w) \in \mathcal{V} \times \mathcal{V}:\left|x_{v} y_{w}\right| \leq(1 / n) \sqrt{T / n}\right\}$ to be the set of light couples. Its complement $\mathcal{L}^{\mathrm{c}} \triangleq \mathcal{V} \backslash \mathcal{L}$ will be called the set of heavy couples. Furthermore define $\mathcal{K} \triangleq\left(\Gamma^{\mathrm{c}} \times \mathcal{V}\right) \cup\left(\mathcal{V} \times \Gamma^{\mathrm{c}}\right)$. Using the triangle inequality, we bound

$$
\left|x^{\mathrm{T}}\left(A_{\Gamma}-M\right) y\right| \leq F_{1}(x, y)+F_{2}(x, y)+F_{3}(x, y)
$$

where $F_{1}(x, y) \triangleq\left|\sum_{(v, w) \in \mathcal{K} \cap \mathcal{L}} x_{v} A_{v w} y_{w}\right|, F_{2}(x, y) \triangleq \mid \sum_{(v, w) \in \mathcal{L}} x_{v} A_{v w} y_{w}-$ $x^{\mathrm{T}} M y \mid$, and $F_{3}(x, y) \triangleq\left|\sum_{(v, w) \in \mathcal{L}^{\mathrm{c}}} x_{v}\left(A_{\Gamma}\right)_{v w} y_{w}\right|$. Next we bound each of these terms individually.

Step 3a: Exponential concentration of $F_{1}(x, y)$.

imsart-aos ver. 2014/10/16 file: JS_AP_SY_CBMC__arXiv.tex date: July 31, 2019 


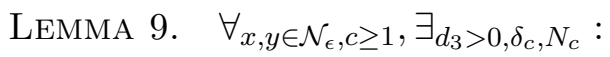

$$
\mathbb{P}\left[F_{1}(x, y) \geq \delta_{c} \sqrt{T / n}\right] \leq \mathrm{e}^{\left(\ln 2-d_{3} c\right) n}, \quad \forall_{n>N_{c}} .
$$

Proof. Let $x, y \in \mathcal{N}_{\epsilon}$. Define $\mathcal{M}_{n} \triangleq\{\mathcal{S} \subseteq \mathcal{V}:|\mathcal{S}|=\lfloor n \exp (-T / n$. $\ln (T / n))\rfloor\}$, and bound

$$
n \sqrt{\frac{n}{T}} F_{1}(x, y) \stackrel{(\mathrm{i})}{\leq}\left|\sum_{(v, w) \in \mathcal{K} \cap \mathcal{L}} A_{v w}\right| \leq 2 \hat{N}_{\mathcal{V}, \Gamma^{\mathrm{c}}} \leq 2 \max _{\mathcal{S} \in \mathcal{M}_{n}}\left\{\hat{N}_{\mathcal{V}, \mathcal{S}}\right\}
$$

by using (i) $\mathcal{L}$ 's definition.

We next show that the r.h.s. of $(123)$ is $O_{\mathbb{P}}(n)$. For some sufficiently large constant $c$, we obtain (i) by Boole's inequality and the fact that $N_{\mathcal{V}, \mathcal{S}}=O\left(n(n / T)^{T / n-1}\right)=o(n)$ since $\mathcal{S} \in \mathcal{M}_{n}$ and $T=\omega(n)$ and (ii) by the observation that the number of subsets of $\mathcal{V}$ is less than $2^{n}$ and (42) that

$\mathbb{P}\left[\max _{\mathcal{S} \in \mathcal{M}_{n}}\left\{\hat{N}_{\mathcal{V}, \mathcal{S}}\right\} \geq c n\right] \stackrel{(\text { i) }}{\leq} \sum_{\mathcal{S} \in \mathcal{M}_{n}} \mathbb{P}\left[\left|\hat{N}_{\mathcal{V}, \mathcal{S}}-N_{\mathcal{V}, \mathcal{S}}\right| \geq c n-o(n)\right] \stackrel{\text { (ii) }}{\leq} 2^{n} \mathrm{e}^{-d_{3} c n} \rightarrow 0$.

This proves Lemma 9 .

Step 3b: Exponential concentration of $F_{2}(x, y)$.

LEMMA 10. $\quad \forall_{x, y \in \mathcal{N}_{\epsilon}, \delta>0}: \mathbb{P}\left[F_{2}(x, y) \geq \delta f_{n}\right] \leq \mathrm{e}^{\left(p_{\max }-\frac{\delta}{2} \sqrt{\ln \frac{T}{n}}\right) n}, \forall_{n>0}$.

Proof. Let $x, y \in \mathcal{N}_{\varepsilon}, a_{n}>0$, and $\lambda>0$. Markov's inequality implies $(125)$

$$
\mathbb{P}\left[\sum_{(v, w) \in \mathcal{L}} x_{v} A_{v w} y_{w}-x^{\mathrm{T}} M y \geq a_{n} \sqrt{\frac{T}{n}}\right] \leq \frac{\mathbb{E}\left[\exp \left(\lambda \sum_{(v, w) \in \mathcal{L}} x_{v} A_{v w} y_{w}\right)\right]}{\exp \left(\lambda a_{n} \sqrt{T / n}+x^{\mathrm{T}} M y\right)} .
$$

We start by bounding the numerator. Prepare for a folding argument by noting that $\hat{N}_{v w}(s) \in\{0,1\}$ and $\sum_{v, w} \hat{N}_{v w}(s)=1$ for $s \geq 0$. This implies that $\mathbb{P}\left[\hat{N}_{r u}(s)=1 \mid \mathcal{F}_{s-2}\right]=\bar{N}_{r u}(s)$. Hence we calculate for $s \geq 0$ that $\mathbb{E}\left[\mathrm{e}^{\lambda \sum_{(v, w) \in \mathcal{L}} x_{v} \hat{N}_{v w}(s) y_{w}} \mid \mathcal{F}_{s-2}\right]=\sum_{(r, u) \in \mathcal{V}^{2}}\left(\mathbb{1}[r, u \notin \mathcal{L}]+\mathbb{1}[r, u \in \mathcal{L}] \mathrm{e}^{\lambda x_{u} y_{r}}\right) \bar{N}_{r u}(s)$

$$
=\sum_{(r, u) \in \mathcal{L}^{c}} \bar{N}_{r u}(s)+\sum_{(r, u) \in \mathcal{L}^{c}} \mathrm{e}^{\lambda x_{r} y_{u}} \bar{N}_{r u}(s)=1+\sum_{(v, w) \in \mathcal{L}^{c}} \bar{N}_{v w}(s)\left(\mathrm{e}^{\lambda x_{v} y_{w}}-1\right) .
$$


We are now in place to use the tower property to fold up the numerator backwards through time. Starting from $t=\lceil T / 2\rceil-1$, we calculate

$$
\begin{aligned}
& \mathbb{E}\left[\exp \left(\lambda \sum_{(v, w) \in \mathcal{L}} x_{v} A_{v w} y_{w}\right)\right]=\mathbb{E}\left[\prod_{t=0}^{\lceil T / 2\rceil-1} \exp \left(\lambda \sum_{(v, w) \in \mathcal{L}} x_{v} \hat{N}_{v w}(2 t) y_{w}\right)\right]= \\
& (127) \\
& \mathbb{E}\left[\mathbb{E}\left[\mathrm{e}^{\lambda \sum_{(v, w) \in \mathcal{L}} x_{v} \hat{N}_{v w}(2\lceil T / 2\rceil-2) y_{w}} \mid \mathcal{F}_{2\lceil T / 2\rceil-4}\right] \prod_{t=0}^{\lceil T / 2\rceil-2} \mathrm{e}^{\lambda \sum_{(v, w) \in \mathcal{L}} x_{v} \hat{N}_{v w}(2 t) y_{w}}\right],
\end{aligned}
$$

and then repeat the argument for $t=\lceil T / 2\rceil-2, \ldots$, et cetera. This brings us to (i) below, and we now use the elementary bounds (ii) $e^{x} \leq 1+x+2 x^{2}$ for $|x| \leq 1 / 2$ and (iii) $1+x \leq e^{x}$ to obtain

$$
\begin{aligned}
& \mathrm{e}^{\lambda a_{n} \sqrt{\frac{T}{n}}} \mathbb{P}\left[\sum_{(v, w) \in \mathcal{L}} x_{v} A_{v w} y_{w}-x^{\mathrm{T}} M y \geq a_{n} \sqrt{\frac{T}{n}}\right] \\
& \stackrel{\text { i) }}{\leq} \mathrm{e}^{-\lambda x^{\mathrm{T}} M y_{\mathbb{E}}}\left[\prod_{t=0}^{\lceil T / 2\rceil-1}\left(1+\sum_{(v, w) \in \mathcal{L}} \bar{N}_{v w}(2 t)\left(\mathrm{e}^{\lambda x_{v} y_{w}}-1\right)\right)\right] \\
& \left.\stackrel{\text { (ii) }}{\leq} \mathrm{e}^{-\lambda x^{\mathrm{T}} M y} \mathbb{E}\left[\prod_{t=0}^{\lceil T / 2\rceil-1}\left(1+\sum_{(v, w) \in \mathcal{L}} \bar{N}_{v w}(2 t)\left(\lambda x_{v} y_{w}+2 \lambda^{2} x_{v}^{2} y_{w}^{2}\right)\right)\right)\right] \\
& \stackrel{(\mathrm{iii})}{\leq} \mathrm{e}^{-\lambda x^{\mathrm{T}} M y_{\mathbb{E}}} \mathbb{E}\left[\exp \left(\sum_{(v, w) \in \mathcal{L}} M_{v w}\left(\lambda x_{v} y_{w}+2 \lambda^{2} x_{v}^{2} y_{w}^{2}\right)\right)\right] \text {. }
\end{aligned}
$$

We next bound the r.h.s. of (128). Use a contradiction argument to note that since $\sum_{v \in \mathcal{V}} \sum_{w \in \mathcal{V}} x_{v}^{2} y_{w}^{2}=1$ and $\left|x_{v} y_{w}\right|>(1 / n) \sqrt{T / n}$ for all $(v, w) \in \mathcal{L}^{\mathrm{c}}$, we must have $\sum_{(v, w) \in \mathcal{L}^{\mathrm{c}}}\left|x_{v} y_{w}\right| \leq n \sqrt{n / T}$. Specify $\lambda=\frac{1}{2} n \sqrt{n / T}$. Together with the bound $M_{v w} \leq p_{\max } T / n^{2}$ for all $v, w$, we obtain

$$
\sum_{(v, w) \in \mathcal{L}} M_{v w} \lambda x_{v} y_{w}-\lambda x^{\mathrm{T}} M y=-\sum_{(v, v) \in \mathcal{L}^{\mathrm{c}}} \lambda M_{v w} x_{v} y_{w} \leq \frac{n}{2} p_{\max }
$$

Additionally since $\sum_{v \in \mathcal{V}} \sum_{w \in \mathcal{V}} x_{v}^{2} y_{w}^{2}=1$, it follows that

$$
\sum_{(v, w) \in \mathcal{L}} M_{v w} 2 \lambda^{2} x_{v}^{2} y_{w}^{2} \leq \frac{n}{2} p_{\max }
$$

Finally, by combining (128)-(130), we obtain

$$
\mathbb{P}\left[\sum_{(v, w) \in \mathcal{L}} x_{v} A_{v w} y_{w}-x^{\mathrm{T}} M y \geq a_{n} \sqrt{\frac{T}{n}}\right] \leq \exp \left(n p_{\max }-\frac{a_{n}}{2} n\right) .
$$

imsart-aos ver. 2014/10/16 file: JS_AP_SY_CBMC_arXiv.tex date: July 31, 2019 
This proves Lemma 10.

Step 3c: Discrepancy property for $F_{3}(x, y)$. We extend the arguments in [31] as follows. First, we introduce the quantity $e(\mathcal{A}, \mathcal{B}) \triangleq \sum_{x \in \mathcal{A}} \sum_{y \in \mathcal{B}}\left(A_{\Gamma}\right)_{x, y}$. Next, we say that the random variable $A_{\Gamma}$ satisfies the discrepancy property if there exist constants $c_{2}, c_{3}>0$ such that for every $\mathcal{A}, \mathcal{B} \subset \mathcal{V}$ one of the following holds:

(i) $\frac{e(\mathcal{A}, \mathcal{B}) n^{2}}{|\mathcal{A}||\mathcal{B}| T} \leq c_{2} \ln \frac{T}{n}$

(ii) $e(\mathcal{A}, \mathcal{B}) \ln \frac{e(\mathcal{A}, \mathcal{B}) n^{2}}{|\mathcal{A}||\mathcal{B}| T} \leq c_{3} \max \{|\mathcal{A}|,|\mathcal{B}|\} \ln \frac{n}{\max \{|\mathcal{A}|,|\mathcal{B}|\}}$.

We now prove that the discrepancy property provides an absolute bound on $F_{3}(x, y)$, and that it holds with high probability.

Lemma 11. If $A_{\Gamma}$ satisfies the discrepancy property, it holds that

$$
\exists_{c>0}: F_{3}(x, y) \leq c f_{n}, \quad \forall x, y \in \mathcal{N}_{\epsilon} .
$$

Proof. This is explained in [31]. This is Remark 4.5 of [35].

Lemma 12. The random variable $A_{\Gamma}$ satisfies the discrepancy property with probability of at least $1-1 / n$.

Proof. Let $\mathcal{A}, \mathcal{B} \subset \mathcal{V}$ be two subsets such that $|\mathcal{A}| \leq b$ w.l.o.g. In these next two paragraphs, we temporarily let $a=|\mathcal{A}|, b=b$ to declutter notation. Also let $c_{2}, c_{3}$ be two large constants (how large will be sufficient will become clear in a moment). We can now distinguish two cases:

Case 1: $b \geq n / 5$. The trimming step ensures that $e(v, \mathcal{V})=O(T / n)$ for all $v \in \mathcal{V}$. We therefore have in this case that $e(\mathcal{A}, \mathcal{B}) \leq c_{2} a b T / n^{2}$ for a sufficiently large constant $c_{2}$.

Case 2: $b \leq n / 5$. For this case, define the quantity $\eta(a, b)=\max \left\{\eta^{0}\right.$, $\left.\left(c_{2} a b T \ln (T / n)\right) / n^{2}\right\}$ with $\eta^{0}$ the constant that satisfies the relation $\eta^{0}$. $\ln \left(\left(\eta^{0} n^{2}\right) /(a b T)\right)=c_{3} b \ln (n / b)$. If all pairs of subsets $\mathcal{A}, \mathcal{B} \subset \mathcal{V}$ satisfy $e(\mathcal{A}, \mathcal{B}) \leq \eta(a, b)$, the discrepancy property holds. It therefore suffices to show that $e(\mathcal{A}, \mathcal{B}) \leq \eta(a, b)$ with high probability for all $\mathcal{A}, \mathcal{B} \subset \mathcal{V}$.

We will first quantify the probability that $e(\mathcal{A}, \mathcal{B}) \leq \eta(a, b)$ for any arbitrary subsets $\mathcal{A}, \mathcal{B} \subset \mathcal{V}$. Using Markov's inequality, we obtain

$$
\begin{aligned}
& \mathbb{P}[e(\mathcal{A}, \mathcal{B})>\eta(a, b)] \leq \inf _{h \geq 0} \frac{\mathbb{E}[\exp (h \cdot e(\mathcal{A}, \mathcal{B}))]}{\exp (h \cdot \eta(a, b))} \\
& \leq \inf _{h \geq 0} \frac{\prod_{t=1}^{\lceil T / 2\rceil-1}\left(1+\frac{a b p_{\max }}{n^{2}} \mathrm{e}^{h}\right)}{\exp (h \cdot \eta(a, b))} \leq \inf _{h \geq 0} \frac{\prod_{t=1}^{\lceil T / 2\rceil-1} \exp \left(\frac{a b p_{\max }}{n^{2}} \mathrm{e}^{h}\right)}{\exp (h \cdot \eta(a, b))}
\end{aligned}
$$

imsart-aos ver. 2014/10/16 file: JS_AP_SY_CBMC__arXiv.tex date: July 31, 2019 


$$
\begin{aligned}
& \leq \inf _{h \geq 0} \exp \left(\frac{a b p_{\max } T}{2 n^{2}} \mathrm{e}^{h}-h \eta(a, b)\right) \\
& \leq \exp \left(-\eta(a, b)\left(\ln \frac{2 n^{2} \eta(a, b)}{a b p_{\max } T}-1\right)\right),
\end{aligned}
$$

where, for the last inequality, we specify $\left.h=\ln \left(2 n^{2} \eta(a, b)\right) /\left(a b p_{\max } T\right)\right)$.

As a last step, we compute the expected number of pairs $\mathcal{A}, \mathcal{B} \subset \mathcal{V}$ such that $e(\mathcal{A}, \mathcal{B})>\eta(|\mathcal{A}|,|\mathcal{B}|)$. The number of possible pairs of sets $\mathcal{A}$ and $\mathcal{B}$ such that $|\mathcal{A}|=a$ and $|\mathcal{B}|=b$ is $\left(\begin{array}{l}n \\ a\end{array}\right)\left(\begin{array}{l}n \\ b\end{array}\right)$. Hence using (132),

$$
\begin{aligned}
& \mathbb{E}[|\{(\mathcal{A}, \mathcal{B})|e(\mathcal{A}, \mathcal{B})>\eta(|\mathcal{A}|,|\mathcal{B}|),| \mathcal{A}|=a,| \mathcal{B} \mid=b, \mathcal{A}, \mathcal{B} \subset \mathcal{V}\}|] \\
& \leq\left(\begin{array}{c}
n \\
a
\end{array}\right)\left(\begin{array}{c}
n \\
b
\end{array}\right) \max _{\mathcal{A}, \mathcal{B} \subset \mathcal{V} \text { s.t. }|\mathcal{A}|=a,|\mathcal{B}|=b} \mathbb{P}[e(\mathcal{A}, \mathcal{B})>\eta(a, b)] \\
& \stackrel{\text { (i) }}{\leq}\left(\frac{n \mathrm{e}}{b}\right)^{2 b} \max _{\mathcal{A}, \mathcal{B} \subset \mathcal{V} \text { s.t. }|\mathcal{A}|=a,|\mathcal{B}|=b} \mathbb{P}[e(\mathcal{A}, \mathcal{B})>\eta(a, b)] \\
& \stackrel{\text { (ii) }}{\leq} \exp \left(4 b \ln \frac{n}{b}-\eta(a, b)\left(\ln \frac{2 n^{2} \eta(a, b)}{a b p_{\max } T}-1\right)\right) \\
& \stackrel{\text { (iii) }}{\leq} \exp \left(-3 \ln n+7 b \ln \frac{n}{b}-\eta(a, b)\left(\ln \frac{2 n^{2} \eta(a, b)}{a b p_{\max } T}-1\right)\right) \\
& \stackrel{\text { (iv) }}{\leq} \exp \left(-3 \ln n+7 b \ln \frac{n}{b}-\frac{\eta(a, b)}{2} \ln \frac{2 n^{2} \eta(a, b)}{a b p_{\max } T}\right) \stackrel{(\mathrm{v})}{\leq} \frac{1}{n^{3}} .
\end{aligned}
$$

Here, we have used that (i,ii) $a \leq b \leq n / 5$, and (iii) that $-3 b \ln (n / b) \leq$ $-3 \ln n$ on the interval $b \in[1, n / 5]$. Inequality (iv) follows from $n^{2} \eta(a, b) /(a b T)$ $\geq c_{2} \ln T / n$ and the fact that $\ln x-1 \geq \frac{1}{2} \ln x$ for sufficiently large $x$, and (v) follows from the definition of $\eta(a, b)$ since

$$
7 b \ln \frac{n}{b}-\frac{\eta(a, b)}{2} \ln \frac{2 n^{2} \eta(a, b)}{a b p_{\max } T} \leq 7 b \ln \frac{n}{b}-\frac{c_{3} b}{2} \ln \frac{n}{b}-\frac{\eta^{0}}{2} \ln \frac{2}{p_{\max }} .
$$

Here, we have used specifically that $\eta(a, b) \geq \eta^{0} \geq 0$, that $\eta^{0}$ satisfies $\eta^{0} \ln \left(\eta^{0} n 2 /(a b T)\right)=c_{3} b \ln (n / b)$, and that $2 / p_{\max } \geq 1$. We have therefore shown that for sufficiently large $c_{3}$, when we sum the above inequality for all possible cardinalities $a, b$,

$$
\mathbb{E}\left[\left|\left\{(\mathcal{A}, \mathcal{B}) \mid e(\mathcal{A}, \mathcal{B})>\eta(|\mathcal{A}|,|\mathcal{B}|), \mathcal{A}, \mathcal{B} \subset V_{1} \cap \Gamma\right\}\right|\right] \leq \frac{1}{n} .
$$

We can thus conclude that the discrepancy property holds with probability $1-1 / n$. 
Step 4: Summary. Before putting the results obtained in the previous steps together, we make the following observation. For generic positive random variables $X, X_{1}, \ldots, X_{m}$ with $m<\infty$ satisfying $X \leq \sum_{i=1}^{m} X_{i}, \mathbb{P}[X \geq x] \leq$ $\sum_{i=1}^{m} \mathbb{P}\left[X_{i} \geq x / m\right]$ since

$$
\begin{aligned}
\mathbb{P}[X<x] & \geq \mathbb{P}\left[\sum_{i=1}^{m} X_{i}<x\right] \geq \mathbb{P}\left[\cap_{i=1}^{m}\left\{X_{i}<x / m\right\}\right] \\
& \geq 1-\mathbb{P}\left[\cup_{i=1}^{m}\left\{X_{i} \geq x / m\right\}\right] \geq 1-\sum_{i=1}^{m} \mathbb{P}\left[X_{i} \geq x / m\right]
\end{aligned}
$$

Therefore for any $\epsilon, \delta>0$

$$
\begin{aligned}
\mathbb{P}\left[\left\|A_{\Gamma}-M\right\| \geq \delta f_{n}\right] & \stackrel{(\mathrm{i})}{\leq} \mathbb{P}\left[\max _{x, y \in \mathcal{N}_{\epsilon}}\left|x^{\mathrm{T}}\left(A_{\Gamma}-M\right) y\right| \geq \delta(1-3 \epsilon) f_{n}\right] \\
& \leq \sum_{i=1}^{3} \mathbb{P}\left[\max _{x, y \in \mathcal{N}_{\epsilon}} F_{i}(x, y) \geq \frac{\delta(1-3 \epsilon) f_{n}}{3}\right]
\end{aligned}
$$

where (i) we have used Lemma 8 . We now bound the r.h.s. of (137):

First and second terms (corresponding to $F_{1}$ and $F_{2}$ ). Since we have exponential concentration, we can use the union bound on the $\epsilon$-net for these terms. Applying Lemma 9 with $c>\left(\zeta_{\epsilon}+\ln 2\right) / d_{3}$ when $\delta>3 \delta_{c} /(1-3 \epsilon)$, we obtain

(i) $\mathrm{e}^{\zeta_{\epsilon} n} \mathbb{P}\left[F_{1}(x, y) \geq(\delta / 3)(1-3 \epsilon) f_{n}\right] \leq \mathrm{e}^{\zeta_{\epsilon} n} \mathbb{P}\left[F_{1}(x, y) \geq \delta_{c} \sqrt{T / n}\right]=$ $O\left(\mathrm{e}^{\left(\zeta_{\epsilon}+\ln 2-d_{3} c\right) n}\right) \rightarrow 0$ as $n \rightarrow \infty$.

Using Lemma 10, we find

(ii) $\mathrm{e}^{\zeta_{\epsilon} n} \mathbb{P}\left[F_{2}(x, y) \geq(\delta / 3)(1-3 \epsilon) f_{n}\right] \leq \mathrm{e}^{\left(\zeta_{\epsilon}+p_{\max }-\frac{\delta}{6} \sqrt{\ln \frac{T}{n}}\right) n} \rightarrow 0$ as $n \rightarrow \infty$.

Third term (corresponding to $F_{3}$ ). The third term cannot be bounded in a sufficiently tight manner using the union bound on the $\epsilon$-net. We instead rely on the discrepancy property. Write $\mathcal{D}$ for the event that $A_{\Gamma}$ satisfies the discrepancy property. Using Lemma 11 for sufficiently large $C$, and Lemma 12 , we arrive at

(iii) $\mathbb{P}\left[\max _{x, y \in \mathcal{N}_{\epsilon}} F_{3}(x, y) \geq C f_{n}\right]=\mathbb{P}\left[\max _{x, y \in \mathcal{N}_{\epsilon}} F_{3}(x, y) \geq C f_{n} \mid \mathcal{D}\right] \mathbb{P}[\mathcal{D}]+$ $\mathbb{P}\left[\max _{x, y \in \mathcal{N}_{\epsilon}} F_{3}(x, y) \geq C f_{n} \mid \mathcal{D}^{\mathrm{c}}\right] \mathbb{P}\left[\mathcal{D}^{\mathrm{c}}\right] \leq 0 \cdot \mathbb{P}[\mathcal{D}]+1 \cdot \mathbb{P}\left[\mathcal{D}^{\mathrm{c}}\right] \leq \frac{1}{n}$

Finally, by bounding (137) with (i)-(iii), we obtain the desired conclusion

$$
\text { if } T=\omega(n) \text {, then } \exists_{\delta}: \lim _{n \rightarrow \infty} \mathbb{P}\left[\left\|\sum_{t=0}^{\lceil T / 2\rceil-1}\left(\hat{N}_{\Gamma}(2 t)-\bar{N}(2 t)\right)\right\| \geq \delta f_{n}\right]=0 .
$$

Together with (117) this implies (111).

imsart-aos ver. 2014/10/16 file: JS_AP_SY_CBMC__arXiv.tex date: July 31, 2019 
SM4.2. Proof of Lemma 4. Because (i) $N_{x, y}=T \Pi_{x} P_{x, y}$, and by $P_{x, y}$ 's definition in (4),

$$
\begin{aligned}
& \left\|N_{x, \cdot}-N_{y, \cdot}\right\|_{2}^{2}=\sum_{z \in \mathcal{V}}\left|N_{x, z}-N_{y, z}\right|^{2} \stackrel{(\mathrm{i})}{=} \sum_{z \in \mathcal{V}}\left|T \Pi_{x} P_{x, z}-T \Pi_{y} P_{y, z}\right|^{2} \\
& \stackrel{(4)}{=} T^{2} \sum_{k=1}^{K} \sum_{z \in \mathcal{V}_{k}}\left|\bar{\Pi}_{\sigma(x)} \frac{p_{\sigma(x), k}}{\left|\mathcal{V}_{k}\right|-\mathbb{1}[\sigma(x)=k]}-\bar{\Pi}_{\sigma(y)} \frac{p_{\sigma(y), k}}{\left|\mathcal{V}_{k}\right|-\mathbb{1}[\sigma(y)=k]}\right|^{2}
\end{aligned}
$$

and

$$
\begin{aligned}
& \left\|N_{\cdot, x}-N_{\cdot, y}\right\|_{2}^{2}=\sum_{z \in \mathcal{V}}\left|N_{z, x}-N_{z, y}\right|^{2} \stackrel{(\mathrm{i})}{=} \sum_{z \in \mathcal{V}}\left|T \Pi_{z} P_{z, x}-T \Pi_{z} P_{z, y}\right|^{2} \\
& \stackrel{(4)}{=} T^{2} \sum_{k=1}^{K} \sum_{z \in \mathcal{V}_{k}}\left|\bar{\Pi}_{k} \frac{p_{k, \sigma(x)}}{\left|\mathcal{V}_{\sigma(x)}\right|-\mathbb{1}[\sigma(x)=k]}-\bar{\Pi}_{k} \frac{p_{k, \sigma(y)}}{\left|\mathcal{V}_{\sigma(y)}\right|-\mathbb{1}[\sigma(y)=k]}\right|^{2} .
\end{aligned}
$$

Then, we have that

$$
\begin{aligned}
& \left\|N_{x, \cdot}^{0}-N_{y, \|_{2}^{0}}^{2}=\right\| N_{x, \cdot}-N_{y, \cdot}\left\|_{2}^{2}+\right\| N_{\cdot, x}-N_{\cdot, y} \|_{2}^{2} \\
& \quad \frac{T^{2}}{n^{3}} \sum_{k=1}^{K}\left(\left(\frac{\pi_{\sigma(x)} p_{\sigma(x), k}}{\alpha_{k} \alpha_{\sigma(x)}}-\frac{\pi_{\sigma(y)} p_{\sigma(y), k}}{\alpha_{k} \alpha_{\sigma(y)}}\right)^{2}+\left(\frac{\pi_{k} p_{k, \sigma(x)}}{\alpha_{k} \alpha_{\sigma(x)}}-\frac{\pi_{k} p_{k, \sigma(y)}}{\alpha_{k} \alpha_{\sigma(y)}}\right)^{2}\right) \\
& \geq \frac{T^{2}}{n^{3}} D(\alpha, p) .
\end{aligned}
$$

That completes this proof.

SM4.3. Proof of Lemma 5. Recall that for any matrix $A \in \mathbb{R}^{n \times n}$ that $\|A\|_{\mathrm{F}}^{2}=\sum_{i=1}^{n} \sigma_{i}^{2}(A)$, and that for the spectral norm $\|A\|=\max _{i=1, \ldots, n}\left\{\sigma_{i}(A)\right\}$. Because both $\hat{R}$ and $N$ are of rank $K$, the matrix $\hat{R}-N$ is of rank at most $2 K$, and therefore

$$
\left\|\hat{R}^{0}-N^{0}\right\|_{\mathrm{F}}^{2}=2\|\hat{R}-N\|_{\mathrm{F}}^{2} \leq 4 K\|\hat{R}-N\|^{2} .
$$

By the triangle inequality it then follows that

$$
\left\|\hat{R}^{0}-N^{0}\right\|_{\mathrm{F}} \leq 2 \sqrt{K}\left(\left\|\hat{R}-\hat{N}_{\Gamma}\right\|+\left\|\hat{N}_{\Gamma}-N\right\|\right) .
$$

Since $K$ is independent of $n$, we just need to bound $\left\|\hat{R}-\hat{N}_{\Gamma}\right\|$ using $\left\|\hat{N}_{\Gamma}-N\right\|$. From the definition of $\hat{R}$,

$$
\left\|\hat{N}_{\Gamma}-\hat{R}\right\|=\sigma_{K+1}\left(\hat{N}_{\Gamma}\right) .
$$

Since the rank of $N$ is at most $K$, Weyl's theorem gives

$$
\sigma_{K+1}\left(\hat{N}_{\Gamma}\right) \leq\left\|\hat{N}_{\Gamma}-N\right\|
$$

The proof is completed after bounding (143) by (144) and (145).

imsart-aos ver. 2014/10/16 file: JS_AP_SY_CBMC__arXiv.tex date: July 31, 2019 
SM4.4. Proof of Lemma 6.

Preliminaries. For notational convenience, let $\bar{N}_{k}^{0} \triangleq\left(1 /\left|\mathcal{V}_{k}\right|\right) \sum_{z \in \mathcal{V}_{k}} N_{z}^{0}$, for $k=1, \ldots, K$. Let $0<a<1 / 2,1+a<b<\infty$ be two constants. Also recall the definitions of neighborhoods in (13):

$$
\mathcal{N}_{x} \triangleq\left\{y \in \mathcal{V} \mid \sqrt{\left\|\hat{R}_{x, \cdot}-\hat{R}_{y, \cdot}\right\|_{2}^{2}+\left\|\hat{R}_{\cdot, x}-\hat{R}_{\cdot, y}\right\|_{2}^{2}} \leq h_{n}\right\} \quad \text { for } \quad x \in \mathcal{V} .
$$

Note that in $(13)$ we specified $h_{n}=(1 / n) \cdot(T / n)^{3 / 2}(\ln (T / n))^{4 / 3}$, while in this proof we assume instead that $h_{n}$ satisfies $\omega\left(f_{n}^{2} / n\right)=h_{n}^{2}=o\left(T^{2} / n^{3}\right)$.

Approach. We show that for any $0<a<1 / 2$ the recursive algorithm in (14) will (for sufficiently large $n, T$ ) give centers $z_{1}^{*}, \ldots, z_{K}^{*}$ satisfying

$$
\left\|\hat{R}_{z_{k}^{*},}^{0}-\bar{N}_{\gamma(k)}^{0}\right\|_{2}<a h_{n} \quad \text { for } \quad k=1, \ldots, K
$$

for some permutation $\gamma$. Assuming (147) holds, one finishes the proof by case checking: let $x \in \mathcal{E}$ be a misclassified state (necessarily $x \notin \mathcal{N}_{z_{\sigma(x)}^{*}}$ ).

Case 1: If $x \in \mathcal{N}_{z_{c}^{*}}$ for some $c \neq \sigma(x)$, we have $\left\|\hat{R}_{x, \cdot}-\bar{N}_{c}^{0}\right\|_{2} \leq(1+a) h_{n}$ by (13) and (147). Together with Lemma 4, this gives the lower bound

$\left\|\hat{R}_{x, \cdot}^{0}-\bar{N}_{\sigma(x)}^{0}\right\|_{2} \stackrel{(\mathrm{i})}{\geq}\left|\left\|\hat{R}_{x, \cdot}^{0}-\bar{N}_{c}^{0}\right\|_{2}-\left\|\bar{N}_{c}^{0}-\bar{N}_{\sigma(x)}^{0}\right\|_{2}\right| \geq \frac{T D^{1 / 2}(\alpha, p)}{n^{3 / 2}}-(1+a) h_{n}$.

By assumption $h_{n}=o\left(T / n^{3 / 2}\right)$, and the result in Lemma 6 follows.

Case 2: Otherwise $x \in\left(\cup_{k=1}^{K} \mathcal{N}_{z_{k}^{*}}\right)^{\mathrm{c}}$, and the algorithm has associated $x$ to the closest (but incorrect) center via (15), i.e., to some cluster $c \neq \sigma(x)$

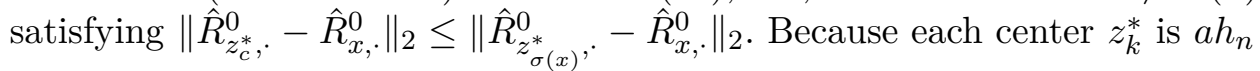
close to its truth $\bar{N}_{k}^{0}$, which themselves are $\Omega\left(T / n^{3 / 2}\right)$ apart, it must be that $\left\|\hat{R}_{x, \cdot}^{0}-\bar{N}_{\sigma(x)}^{0}\right\|_{2}=\Omega\left(T / n^{3 / 2}\right)$.

Proof of (147). To prove (147), we will construct $K$ disjoint sets $\mathcal{C}_{1}, \ldots, \mathcal{C}_{K}$ that satisfy

$$
\exists z \in\left(\cup_{l=1}^{K} \mathcal{C}_{l}\right) \backslash \cup_{l=0}^{k-1} C^{(l)}:\left|\mathcal{N}_{z}\right| \geq\left|\mathcal{C}^{(k)}\right| \geq n \alpha_{k}\left(1-o_{\mathbb{P}}(1)\right) .
$$

Here, the $\left|\mathcal{C}^{(1)}\right| \geq \ldots \geq\left|\mathcal{C}^{(K)}\right|$ denote the order statistic of the sets $\mathcal{C}_{1}, \ldots, \mathcal{C}_{K}$ based on their cardinalities, and $\alpha^{(1)} \geq \ldots \geq \alpha^{(K)}$ denote the order statistic for the cluster concentrations. The existence of sets $\mathcal{C}_{1}, \ldots, \mathcal{C}_{K}$ for which property (149) holds namely implies that it is impossible that any one of the centers $z_{1}^{*}, \ldots, z_{K}^{*}$ provided by the recursion in (14) is an outlier when $n, T$ are sufficiently large. Specifically, we define the sets of cores:

$$
\mathcal{C}_{k} \triangleq\left\{x \in \mathcal{V}_{k} \mid\left\|\hat{R}_{x, \cdot}^{0}-\bar{N}_{k}^{0}\right\|_{2} \leq a h_{n}\right\} \quad \text { for } \quad k=1, \ldots, K,
$$

imsart-aos ver. 2014/10/16 file: JS_AP_SY_CBMC__arXiv.tex date: July 31, 2019 


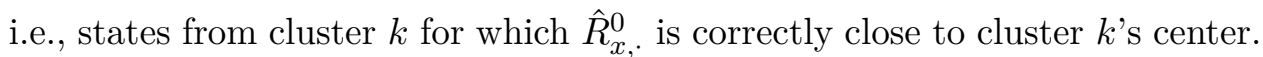
We also define the set of outliers:

$$
\mathcal{O} \triangleq\left\{x \in \mathcal{V} \mid\left\|\hat{R}_{x, \cdot}^{0}-\bar{N}_{k}^{0}\right\|_{2} \geq b h_{n} \text { for all } k=1, \ldots, K\right\},
$$

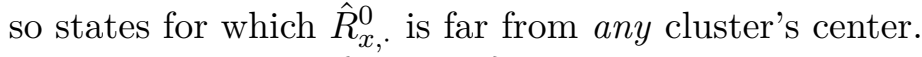

Let $x \in \mathcal{O}, k \in\{1, \ldots, K\}$ and $y \in \mathcal{C}_{k}$. The situation is schematically depicted in Figure 9. By centering and then applying the reverse triangle inequality, we find

$$
\left\|\hat{R}_{x, \cdot}^{0}-\hat{R}_{y, \cdot}^{0}\right\|_{2} \geq\left|\left\|\hat{R}_{x, \cdot}^{0}-\bar{N}_{k}^{0}\right\|_{2}-\left\|\hat{R}_{y, \cdot}^{0}-\bar{N}_{k}^{0}\right\|_{2}\right| .
$$

Since $x \in \mathcal{O}$ and $y \in \mathcal{C}_{k}$, it follows that $\left\|\hat{R}_{x, \cdot}^{0}-\hat{R}_{y,}^{0}\right\|_{2} \geq(b-a) h_{n}$. Furthermore $b-a>1$, implying that $y \notin \mathcal{N}_{x}$. We have shown that $\mathcal{N}_{x} \cap\left(\cup_{k=1}^{K} \mathcal{C}_{k}\right)=\emptyset$ for all $x \in \mathcal{O}$. Consequentially:

(a) for any $x \in \mathcal{O},\left|\mathcal{N}_{x}\right| \leq\left|\left(\cup_{k=1}^{K} \mathcal{C}_{k}\right)^{\mathrm{c}}\right|$ since $\mathcal{N}_{x} \subseteq\left(\cup_{k=1}^{K} \mathcal{C}_{k}\right)^{\mathrm{c}}$.

Furthermore:

(b) for any $y \in \cup_{k=1}^{K} \mathcal{C}_{k}, \mathcal{C}_{\sigma(y)} \subseteq \mathcal{N}_{y}$ since $a<1 / 2$,

(c) for $k \neq l$ and sufficiently large $n, T, \mathcal{C}_{k} \cap \mathcal{C}_{l}=\emptyset$ since $h_{n}=o\left(T / n^{3 / 2}\right)$.

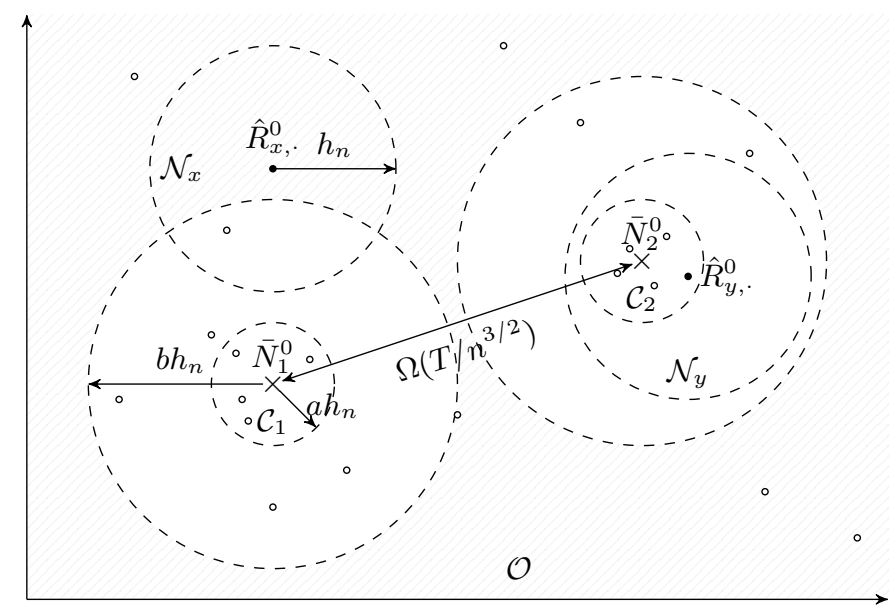

Fig 9: Schematic depictions of cores $\mathcal{C}_{1}, \mathcal{C}_{2}$ for $K=2$, the set of outliers $\mathcal{O}$ (shaded area), and neighborhoods $\mathcal{N}_{x}, \mathcal{N}_{y}$ for some $x \in \mathcal{O}$ and $y \in \mathcal{C}_{2}$.

We now provide estimates for the sizes of the sets involved in $(\mathrm{a}-\mathrm{c})$. By (ii) Lemma 5, and (150),

$$
16 K\left\|\hat{N}_{\Gamma}-N\right\|^{2} \stackrel{(\mathrm{ii})}{\geq}\left\|\hat{R}^{0}-N^{0}\right\|_{\mathrm{F}}^{2}=\sum_{x \in \mathcal{V}}\left\|\hat{R}_{x, \cdot}^{0}-\bar{N}_{\sigma(x)}^{0}\right\|_{2}^{2}
$$

imsart-aos ver. 2014/10/16 file: JS_AP_SY__CBMC_-arXiv.tex date: July 31, 2019 


$$
\geq\left|\left(\cup_{k=1}^{K} \mathcal{C}_{k}\right)^{\mathrm{c}}\right| \min _{x \in\left(\cup_{k=1}^{K} \mathcal{C}_{k}\right)^{\mathrm{c}}}\left\{\left\|\hat{R}_{x, \cdot}^{0}-\bar{N}_{\sigma(x)}^{0}\right\|_{2}^{2}\right\} \stackrel{(150)}{\geq}\left|\left(\cup_{k=1}^{K} \mathcal{C}_{k}\right)^{\mathrm{c}}\right| a^{2} h_{n}^{2}
$$

Rearrange to conclude that $\left|\left(\cup_{k=1}^{K} \mathcal{C}_{k}\right)^{\mathrm{c}}\right|=O_{\mathbb{P}}\left(f_{n}^{2} / h_{n}^{2}\right)=o_{\mathbb{P}}(n)$. Similarly for any $k \in\{1, \ldots, K\}, 16 K\left\|\hat{N}_{\Gamma}-N\right\|^{2} \geq\left|\mathcal{C}_{k}^{\mathrm{c}} \cap \mathcal{V}_{k}\right| a^{2} h_{n}^{2}$ such that $\left|\mathcal{C}_{k}\right|=$ $\left|\mathcal{V}_{k}\right|-\left|\mathcal{C}_{k}^{\mathrm{c}} \cap \mathcal{V}_{k}\right| \geq n \alpha_{k}-O_{\mathbb{P}}\left(f_{n}^{2} / h_{n}^{2}\right)=n \alpha_{k}\left(1-o_{\mathbb{P}}(1)\right)$. Together with (a-c), this establishes the existence of $\mathcal{C}_{1}, \ldots, \mathcal{C}_{K}$ such that (149) holds.

\section{SM5. Proofs of Chapter 7.}

SM5.1. Bounding the size of $\mathcal{H}^{\mathrm{c}}=\mathcal{V} \backslash \mathcal{H}$ (Proof of Proposition 9). First note that the number of states not in $\Gamma$ (obtained after the trimming process) is negligible, i.e., $n \exp \left(-\frac{T}{n} \ln \left(\frac{T}{n}\right)\right)$. We then upper bound the number of states that do not satisfy (H1). Let $x \in \mathcal{V}_{i}$. If $x$ does not satisfy (H1), there exists $j \neq i$ such that $\hat{I}_{i, j}(x)<\frac{T}{2 n} I(\alpha, p)$, where

$$
\hat{I}_{i, j}(x) \triangleq \sum_{k=1}^{K}\left(\hat{N}_{x, \mathcal{V}_{k}} \ln \frac{p_{i, k}}{p_{j, k}}+\hat{N}_{\mathcal{V}_{k}, x} \ln \frac{p_{k, i} \alpha_{j}}{p_{k, j} \alpha_{i}}\right)+\left(\frac{\hat{N}_{\mathcal{V}_{j}, \mathcal{V}}}{\alpha_{j} n}-\frac{\hat{N}_{\mathcal{V}_{i}, \mathcal{V}}}{\alpha_{i} n}\right) .
$$

We have $\mathbb{E}\left[\hat{I}_{i, j}(x)\right]=\frac{T}{n} I_{i, j}(\alpha, p)$ where $I_{i, j}(\alpha, p)$ is the quantity involved in the definition of $I(\alpha, p)(10)$. In particular, $\mathbb{E}\left[\hat{I}_{i, j}(x)\right] \geq \frac{T}{n} I(\alpha, p)$. Hence, $x$ does not satisfy (H1) implies that for some $j \neq i, \hat{I}_{i, j}(x)<\frac{T}{2 n} I(\alpha, p)$ and $\mathbb{E}\left[\hat{I}_{i, j}(x)\right] \geq \frac{T}{n} I(\alpha, p)$. Using concentration results for the BMC $\S S M 1$ and more precisely $(43)$, this event happens with probability at most $\exp (-C$ $\left.\frac{T}{n} I(\alpha, p)\right)$ with $C=\alpha_{\min }^{2} /\left(720 \eta^{3} \alpha_{\max }^{2}\right)$. Hence, the expected number of states not satisfying (H1) is bounded by $n \exp \left(-C \frac{T}{n} I(\alpha, p)\right)$. From there, using Markov inequality, we obtain that the number of states not satisfying (H1) does not exceed $n \exp \left(-C \frac{T}{n} I(\alpha, p)\right)$ with high probability.

Next, we prove the following intermediate claim:

Lemma 13. Define $s \triangleq\left\lfloor 2 n \exp \left(-\frac{\alpha_{\min }^{2}}{720 \eta^{3} \alpha_{\max }^{2}}(T / n) I(\alpha, p)\right)\right\rfloor$. If $s \geq 1$, then with high probability there does not exist a subset $\mathcal{S} \subset \mathcal{V}$ of size $|\mathcal{S}|=s$ such that $\hat{N}_{\mathcal{S}, \mathcal{S}} \geq s \ln (T / n)^{2}$.

Proof. Let $\mathcal{S} \subset \mathcal{V}$ be such that $|\mathcal{S}|=s$ with $s$ as above. We decompose $\hat{N}_{\mathcal{S}, \mathcal{S}}$ as $\hat{N}_{\mathcal{S}, \mathcal{S}}=\hat{N}_{\mathcal{S}, \mathcal{S}}^{(\text {even })}+\hat{N}_{\mathcal{S}, \mathcal{S}}^{(\text {odd })}$ where $\hat{N}_{\mathcal{S}, \mathcal{S}}^{(\text {even })} \triangleq \sum_{t=0}^{\lceil T / 2\rceil-1} \mathbb{1}\left[X_{2 t} \in\right.$ $\left.\mathcal{S}, X_{2 t+1} \in \mathcal{S}\right]$, and similarly $\hat{N}_{\mathcal{S}, \mathcal{S}}^{(\text {odd })} \triangleq \sum_{t=0}^{\lfloor T / 2\rfloor-1} \mathbb{1}\left[X_{2 t+1} \in \mathcal{S}, X_{2 t+2} \in \mathcal{S}\right]$. This decomposition has the same purpose as that in the proofs presented in $\S S M 4.1$, i.e., to get tight bounds on $\hat{N}_{\mathcal{S}, \mathcal{S}}$. More precisely, we have

$\mathbb{P}\left[\hat{N}_{\mathcal{S}, \mathcal{S}}^{(\text {even })} \geq \frac{s}{2} \ln \left(\frac{T}{n}\right)^{2}\right] \stackrel{(\text { i })}{\leq} \inf _{\lambda \geq 0} \frac{\mathbb{E}\left[\mathrm{e}^{\lambda \hat{N}_{\mathcal{S}, \mathcal{S}}^{(\text {even }}}\right]}{\mathrm{e}^{(s / 2) \lambda \ln (T / n)^{2}}} \stackrel{\text { (ii) }}{\leq} \inf _{\lambda \geq 0}^{\lceil T / 2\rceil-1} \prod_{i=1}^{\lceil} \frac{1+(s / n)^{2} p_{\max } \mathrm{e}^{\lambda}}{\mathrm{e}^{(s / 2) \lambda \ln (T / n)^{2}}}$

imsart-aos ver. 2014/10/16 file: JS_AP_SY_CBMC__arXiv.tex date: July 31, 2019 


$$
\begin{aligned}
& \leq \inf _{\lambda \geq 0} \exp \left(\frac{s^{2} p_{\max } T}{n^{2}} \mathrm{e}^{\lambda}-\frac{s}{2} \lambda \ln \left(\frac{T}{n}\right)^{2}\right) \\
& \stackrel{\text { (iii) }}{\leq} \exp \left(-\frac{T}{n} s\left(\frac{1}{2} \ln \frac{T}{n}-\frac{s p_{\max }}{n} \mathrm{e}^{\frac{T / n}{\ln (T / n)}}\right)\right) \stackrel{(\text { iv) }}{\leq} \mathrm{e}^{-\frac{T s \ln (T / n)}{4 n}},
\end{aligned}
$$

for sufficiently large $n, T$. Here, (i) is obtained by applying Markov's inequality. (ii) by directly bounding the transition probabilities - recall that $p_{\max }$ is defined so that $\left(\operatorname{diag}\left(P_{x, \cdot}\right) P\right)_{y, z} \leq p_{\max } / n^{2}$ for all $x, y, z \in \mathcal{V}$, see $\S$ SM4.1 for details. (iii) is obtained by specifying $\lambda=(T / n) /(\ln (T / n))$. Finally to get (iv), we used the fact that $n / s \geq \exp ((T / n) /(\ln (T / n))$. Analogously, one can prove that

$$
\mathbb{P}\left[\hat{N}_{\mathcal{S}, \mathcal{S}}^{\text {(odd) }} \geq \frac{s}{2} \ln \left(\frac{T}{n}\right)^{2}\right] \leq \exp \left(-\frac{T s \ln (T / n)}{4 n}\right) .
$$

Because the number of subsets $\mathcal{S} \subset \mathcal{V}$ of size $s$ satisfies $\left(\begin{array}{l}n \\ s\end{array}\right) \leq(\mathrm{e} n / s)^{s}$ we deduce using (154) and (155) that

$$
\begin{aligned}
& \mathbb{E}\left[\left|\left\{\mathcal{S}: \hat{N}_{\mathcal{S}, \mathcal{S}} \geq s \ln \left(\frac{T}{n}\right)^{2},|\mathcal{S}|=s\right\}\right|\right] \\
& \leq \mathbb{E}\left[\left|\left\{\mathcal{S}: \hat{N}_{\mathcal{S}, \mathcal{S}}^{\text {(even })} \geq \frac{s}{2} \ln \left(\frac{T}{n}\right)^{2},|\mathcal{S}|=s\right\}\right|\right] \\
& \quad+\mathbb{E}\left[\left|\left\{\mathcal{S}: \hat{N}_{\mathcal{S}, \mathcal{S}}^{\text {(odd })} \geq \frac{s}{2} \ln \left(\frac{T}{n}\right)^{2},|\mathcal{S}|=s\right\}\right|\right] \\
& \leq 2\left(\frac{\mathrm{e} n}{s}\right)^{s} \mathrm{e}^{-\frac{T s \ln (T / n)}{4 n}}=2 \mathrm{e}^{-s\left(\frac{T \ln (T / n)}{4 n}-\ln \frac{\mathrm{e} n}{s}\right)} \leq 2 \mathrm{e}^{-\frac{T s \ln (T / n)}{8 n}}
\end{aligned}
$$

for sufficiently large $n, T$. Using Markov's inequality, we can now conclude that with high probability there does not exist a subset $\mathcal{S} \subset \mathcal{V}$ of size $|\mathcal{S}|=s$ such that $\hat{N}_{\mathcal{S}, \mathcal{S}} \geq s \ln (T / n)^{2}$.

We then complete the proof of Proposition 9 applying the following argument. Consider this iterative construction: start with the set $Z(0)$ of all states in $\Gamma$ that do not satisfy (H1). The $t$-th iteration consists of adding to $Z(t-1)$ a state $v$ not satisfying $(\mathrm{H} 2)$ written w.r.t. $Z(t-1)$, i.e., $\hat{N}_{v, Z(t-1)}+$ $\hat{N}_{Z(t-1), v}>2 \ln \left((T / n)^{2}\right)$. If such a state does not exist, the construction ends. Let $Z\left(t^{*}\right)$ be the final set: $t^{*}$ is the number of iterations before the construction stops. By definition of $\mathcal{H}$, the size of $\mathcal{V} \backslash Z\left(t^{*}\right)$ is smaller than that of $\mathcal{H}$, and thus $\left|\mathcal{H}^{\mathrm{c}}\right| \leq\left|Z\left(t^{*}\right)\right|=|Z(0)|+t^{*}$. Note that with high probability $|Z(0)| \leq s / 2$.

Finally, we show that $|Z(0)|+t^{*} \leq s$ with high probability when $|Z(0)| \leq$ $s / 2$. We first consider the case when $s=0$, i.e., $Z(0)=\emptyset$. When $Z(0)=\emptyset$, $t^{*}=0$ since $\hat{N}_{x, \emptyset}+\hat{N}_{\emptyset, x}=0<2 \ln \left((T / n)^{2}\right)$ for every $x \in \mathcal{V}$. Therefore, $\left|\mathcal{H}^{\mathrm{c}}\right|=$ 
$s$. We then consider the case when $s \geq 1$. By construction, we have $\hat{N}_{Z(t), Z(t)}$ $\geq 2 t \ln (T / n)^{2}$ and $|Z(t)| \leq s / 2+t$ because of the sequence generating rule. Further observe that $Z\left(t^{*}\right)$ is such that for all $x \notin Z\left(t^{*}\right), x$ satisfies (H1) and (H2) written w.r.t. $Z\left(t^{*}\right)$. Assume that $t^{*} \geq s / 2$. When $t=s / 2$, the set $Z(s / 2)$ satisfies that $\hat{N}_{Z(s / 2), Z(s / 2)} \geq s \ln (T / n)^{2}$ and $|Z(s / 2)| \leq s$. From Lemma 13, however, there does not exist a subset $\mathcal{S} \subset \mathcal{V}$ of size $|\mathcal{S}|=s$ such that $\hat{N}_{\mathcal{S}, \mathcal{S}} \geq s \ln (T / n)^{2}$ with high probability. Therefore, $\left|\mathcal{H}^{\mathrm{c}}\right| \leq\left|Z\left(t^{*}\right)\right| \leq s / 2+t^{*}<s$ with high probability.

\section{SM5.2. Proof of Lemma 7.}

SM5.2.1. Leading order behavior of $E_{1}$. The statement $-E_{1}=\Omega_{\mathbb{P}}(I(\alpha, p)$ $\left.(T / n) e_{n}^{[t+1]}\right)$ is a direct consequence of condition (H1). Specifically, $x \in \mathcal{E}_{\mathcal{H}}^{[t+1]}$ implies that $x \in \mathcal{H}$, and therefore that condition (H1) is satisfied for $x$. Summing condition $(\mathrm{H} 1)$ over all $x \in \mathcal{E}_{\mathcal{H}}^{[t+1]}$ yields the desired result.

SM5.2.2. Leading order behavior of $E_{2}$. By assumption, we can bound all the ratios of the type $\frac{p_{a, k}}{p_{b, k}}$ or $\frac{p_{k, a}}{p_{k, b}}$ involved in $E_{2}$ by $\eta$. The triangle inequality then yields:

$$
\left|E_{2}\right| \leq \ln \eta\left(\sum_{x \in \mathcal{E}_{\mathcal{H}}^{[t+1]}} \sum_{k=1}^{K}\left|\hat{N}_{x, \hat{\mathcal{V}}_{k}^{[t]}}-\hat{N}_{x, \mathcal{V}_{k}}\right|+\sum_{x \in \mathcal{E}_{\mathcal{H}}^{[t+1]}} \sum_{k=1}^{K}\left|\hat{N}_{\hat{\mathcal{V}}_{k}^{[t]}, x}-\hat{N}_{\mathcal{V}_{k}, x}\right|\right) .
$$

Let us upper bound the first summation in the r.h.s. of (157). Using the triangle inequality and the nonnegativity of entries of $\hat{N}_{x, y}$, we get

$$
\begin{aligned}
\sum_{x \in \mathcal{E}_{\mathcal{H}}^{[t+1]}} \sum_{k=1}^{K}\left|\hat{N}_{x, \hat{\mathcal{V}}_{k}^{[t]}}-\hat{N}_{x, \mathcal{V}_{k}}\right| \leq & \sum_{x \in \mathcal{E}_{\mathcal{H}}^{[t+1]}} \sum_{k=1}^{K}\left|\hat{N}_{x, \hat{\mathcal{V}}_{k}^{[t]} \cap \mathcal{H}}-\hat{N}_{x, \mathcal{V}_{k} \cap \mathcal{H}}\right| \\
& +\sum_{x \in \mathcal{E}_{\mathcal{H}}^{[t+1]}} \sum_{k=1}^{K}\left(\hat{N}_{x, \hat{\mathcal{V}}_{k}^{[t]} \cap \mathcal{H}^{\mathrm{c}}}+\hat{N}_{\left.x, \mathcal{V}_{k} \cap \mathcal{H}^{c}\right)}\right. \\
= & \sum_{x \in \mathcal{E}_{\mathcal{H}}^{[t+1]}} \sum_{k=1}^{K} \mid \hat{N}_{x, \hat{\mathcal{V}}_{k}^{[t]} \cap \mathcal{H}}-\hat{N}_{x, \mathcal{V}_{k} \cap \mathcal{H} \mid}+2 \sum_{x \in \mathcal{E}_{\mathcal{H}}^{[t+1]}} \hat{N}_{x, \mathcal{V} \backslash \mathcal{H}} .
\end{aligned}
$$

To further upper bound the first summation in (158), observe that

$$
\sum_{x \in \mathcal{E}_{\mathcal{H}}^{[t+1]}} \sum_{k=1}^{K}\left|\hat{N}_{x, \hat{\mathcal{V}}_{k}^{[t]} \cap \mathcal{H}}-\hat{N}_{x, \mathcal{V}_{k} \cap \mathcal{H}}\right|=\sum_{x \in \mathcal{E}_{\mathcal{H}}^{[t+1]}} \sum_{k=1}^{K}\left|\sum_{y \in \hat{\mathcal{V}}_{k}^{[t]} \cap \mathcal{H}} \hat{N}_{x, y}-\sum_{y \in \mathcal{V}_{k} \cap \mathcal{H}} \hat{N}_{x, y}\right|
$$

imsart-aos ver. 2014/10/16 file: JS_AP_SY__CBMC_-arXiv.tex date: July 31, 2019 


$$
\begin{aligned}
& =\left.\sum_{x \in \mathcal{E}_{\mathcal{H}}^{[t+1]}} \sum_{k=1}^{K}\right|_{y \in\left(\hat{\mathcal{V}}_{k}^{[t]} \cap \mathcal{H}\right) \backslash\left(\mathcal{V}_{k} \cap \mathcal{H}\right)} \hat{N}_{x, y}-\sum_{y \in\left(\mathcal{V}_{k} \cap \mathcal{H}\right) \backslash\left(\hat{\mathcal{V}}_{k}^{[t]} \cap \mathcal{H}\right)} \hat{N}_{x, y} \mid \\
& \leq \sum_{x \in \mathcal{E}_{\mathcal{H}}^{[t+1]}} \sum_{k=1}^{K} \sum_{y \in\left(\hat{\mathcal{V}}_{k}^{[t]} \cap \mathcal{H}\right) \Delta\left(\mathcal{V}_{k} \cap \mathcal{H}\right)} \hat{N}_{x, y}=2 \sum_{x \in \mathcal{E}_{\mathcal{H}}^{[t+1]}} \sum_{y \in \mathcal{E}_{\mathcal{H}}^{[t]}} \hat{N}_{x, y} .
\end{aligned}
$$

For the second summation in (158), note that since $x \in \mathcal{E}_{\mathcal{H}}^{[t+1]}$ implies that $x \in$ $\mathcal{H}$, it follows from condition (H2) that $\sum_{x \in \mathcal{E}_{\mathcal{H}}^{[t+1]}} \hat{N}_{x, \mathcal{V} \backslash \mathcal{H}} \leq 2\left|\mathcal{E}_{\mathcal{H}}^{[t+1]}\right| \ln \left((T / n)^{2}\right)$.

Now, aside from swapping the indices, the conclusion holds similarly for the second summation in (157). We thus conclude that

$$
\left|E_{2}\right| \leq 2 \ln \eta\left(\hat{N}_{\mathcal{E}_{\mathcal{H}}^{[t+1]}{ }_{\mathcal{E}_{\mathcal{H}}}^{[t]}}+\hat{N}_{\mathcal{E}_{\mathcal{H}}^{[t]}, \mathcal{E}_{\mathcal{H}}^{[t+1]}}+2\left|\mathcal{E}_{\mathcal{H}}^{[t+1]}\right| \ln \left((T / n)^{2}\right)\right)
$$

Next to bound the two first terms of the r.h.s. of (160), we center both terms around their means. Since the Markov chain is in equilibrium by assumption, it holds for the first term that

$$
\begin{aligned}
& \hat{N}_{\mathcal{E}_{\mathcal{H}}^{[t+1]}{ }_{, \mathcal{H}}^{[t]}}=N_{\mathcal{E}_{\mathcal{H}}^{[t+1]}, \mathcal{E}_{\mathcal{H}}^{[t]}}+\hat{N}_{\mathcal{E}_{\mathcal{H}}^{[t+1]}, \mathcal{E}_{\mathcal{H}}^{[t]}}-N_{\mathcal{E}_{\mathcal{H}}^{[t+1]}, \mathcal{E}_{\mathcal{H}}^{[t]}} \\
& \leq \max _{x, y}\left\{T \Pi_{x} P_{x, y}\right\}\left|\mathcal{E}_{\mathcal{H}}^{[t]}\right|\left|\mathcal{E}_{\mathcal{H}}^{[t+1]}\right|+\hat{N}_{\mathcal{E}_{\mathcal{H}}^{[t+1]}, \mathcal{E}_{\mathcal{H}}^{[t]}}-N_{\mathcal{E}_{\mathcal{H}}^{[t+1]}, \mathcal{E}_{\mathcal{H}}^{[t]}} .
\end{aligned}
$$

Applying Lemma 20 presented in §SM6.4, we find that

$$
\hat{N}_{\mathcal{E}_{\mathcal{H}}^{[t+1]}, \mathcal{E}_{\mathcal{H}}^{[t]}}-N_{\mathcal{E}_{\mathcal{H}}^{[t+1]}, \mathcal{E}_{\mathcal{H}}^{[t]}}=1_{\mathcal{E}_{\mathcal{H}}^{[t+1]}}{ }^{\mathrm{T}}(\hat{N}-N) 1_{\mathcal{E}_{\mathcal{H}}^{[t]}} \leq\|\hat{N}-N\| \sqrt{\left|\mathcal{E}_{\mathcal{H}}^{[t]} \| \mathcal{E}_{\mathcal{H}}^{[t+1]}\right|}
$$

The same conclusion holds for $\hat{N}_{\mathcal{E}_{\mathcal{H}}^{[t]}}, \mathcal{E}_{\mathcal{H}}^{[t+1]}-N_{\mathcal{E}_{\mathcal{H}}^{[t]}, \mathcal{E}_{\mathcal{H}}^{[t+1]}}$.

Summarizing, we have shown that

$$
\begin{aligned}
\left|E_{2}\right| \leq 4 \ln \eta(\underbrace{\max _{x, y}\left\{T \Pi_{x} P_{x, y}\right\}\left|\mathcal{E}_{\mathcal{H}}^{[t]}\right|\left|\mathcal{E}_{\mathcal{H}}^{[t+1]}\right|}_{\triangleq F_{1}} \\
+\underbrace{\|\hat{N}-N\| \sqrt{\left|\mathcal{E}_{\mathcal{H}}^{[t]}\right|\left|\mathcal{E}_{\mathcal{H}}^{[t+1]}\right|}}_{\triangleq F_{2}}+\underbrace{\left|\mathcal{E}_{\mathcal{H}}^{[t+1]}\right| \ln \left((T / n)^{2}\right)}_{\triangleq F_{3}}) .
\end{aligned}
$$

Recall that $\Pi_{x} P_{x, y}=O\left(1 / n^{2}\right)$, and hence $F_{1}=O_{\mathbb{P}}\left((T / n)\left(e_{n}^{[t]} / n\right) e_{n}^{[t+1]}\right)$. Next in view of Proposition $7,\|\hat{N}-N\|=O_{\mathbb{P}}(\sqrt{(T / n) \ln (T / n)})$ so that $F_{2}=O_{\mathbb{P}}\left(\sqrt{(T / n) \ln (T / n) e_{n}^{[t]} e_{n}^{[t+1]}}\right) . F_{3}=O_{\mathbb{P}}\left(\ln \left(T^{2} / n^{2}\right) e_{n}^{[t+1]}\right)$ is immediate. This completes the analysis of $E_{2}$. 
SM5.2.3. Leading order behavior of $U$. We write $U=E_{3}+E_{4}$ where $E_{3}=E_{3}^{\text {out }}+E_{3}^{\text {in }}$,

$$
\begin{aligned}
E_{3}^{\text {out }} & =\sum_{x \in \mathcal{E}_{\mathcal{H}}^{[t+1]}} \sum_{k=1}^{K} \hat{N}_{x, \hat{\mathcal{V}}_{k}^{[t]}}\left(\ln \frac{\hat{p}_{\sigma}^{[t+1]}(x), k}{\hat{p}_{\sigma(x), k}}-\ln \frac{p_{\sigma^{[t+1]}(x), k}}{p_{\sigma(x), k}}\right), \\
E_{3}^{\text {in }} & =\sum_{x \in \mathcal{E}_{\mathcal{H}}^{[t+1]}} \sum_{k=1}^{K} \hat{N}_{\hat{\mathcal{V}}_{k}^{[t]}, x}\left(\ln \frac{\hat{p}_{k, \sigma} \hat{p}_{k, \sigma(x)}(x)}{\hat{p}_{k, 1]}}-\ln \frac{p_{k, \sigma}[t+1](x)}{p_{k, \sigma(x)}}\right) .
\end{aligned}
$$

and

$$
E_{4}=\sum_{x \in \mathcal{E}_{\mathcal{H}}^{[t+1]}}\left(\frac{\hat{N}_{\hat{\mathcal{V}}_{\sigma(x)}^{[t]}, \mathcal{V}}}{\left|\hat{\mathcal{V}}_{\sigma(x)}^{[t]}\right|}-\frac{\hat{N}_{\mathcal{V}_{\sigma(x)}, \mathcal{V}}}{\left|\mathcal{V}_{\sigma(x)}\right|}\right)-\sum_{x \in \mathcal{E}_{\mathcal{H}}^{[t+1]}}\left(\frac{\hat{N}_{\hat{\mathcal{V}}_{\sigma}^{[t]}}^{[t+1](x)}, \mathcal{V}}{\left|\hat{\mathcal{V}}_{\sigma^{[t+1]}(x)}^{[t]}\right|}-\frac{\hat{N}_{\mathcal{V}_{\sigma}^{[t+1]}(x)}, \mathcal{V}}{\left|\mathcal{V}_{\sigma^{[t+1]}(x)}\right|}\right) .
$$

LEMMA 14. We have $\left|E_{3}\right|=O_{\mathbb{P}}\left(e_{n}^{[t+1]} \sqrt{\frac{T}{n}} \ln \frac{T}{n}+e_{n}^{[t+1]} \frac{e_{n}^{[t]}}{n} \frac{T}{n} \ln \frac{T}{n}\right)$.

Proof. By the triangle inequality, we have $\left|E_{3}\right| \leq\left|E_{3}^{\text {out }}\right|+\left|E_{3}^{\text {in }}\right|$ with

$$
\begin{aligned}
\left|E_{3}^{\text {out }}\right| & \leq \sum_{x \in \mathcal{E}_{\mathcal{H}}^{[t+1]}} \sum_{k=1}^{K} \hat{N}_{\hat{\mathcal{V}}_{k}^{[t]}, x}\left(\left|\ln \frac{\hat{p}_{k, \sigma^{[t+1]}}(x)}{p_{k, \sigma^{[t+1]}(x)}}\right|+\left|\ln \frac{\hat{p}_{k, \sigma(x)}}{p_{k, \sigma(x)}}\right|\right), \\
\left|E_{3}^{\mathrm{in}}\right| & \leq \sum_{x \in \mathcal{E}_{\mathcal{H}}^{[t+1]}} \sum_{k=1}^{K} \hat{N}_{x, \hat{\mathcal{V}}_{k}^{[t]}}\left(\left|\ln \frac{\hat{p}_{\sigma^{[t+1]}(x), k}}{p_{\sigma^{[t+1]}(x), k}}\right|+\left|\ln \frac{\hat{p}_{\sigma(x), k}}{p_{\sigma(x), k}}\right|\right) .
\end{aligned}
$$

We first bound the summands. From the inequalities $x /(1+x) \leq \ln (1+x)$ $\leq x$ for $x>-1$, it follows that for $a, b=1, \ldots, K$,

$$
\begin{aligned}
& \left|\ln \frac{\hat{p}_{a, b}}{p_{a, b}}\right|=\left|\ln \left(1+\frac{\hat{p}_{a, b}-p_{a, b}}{p_{a, b}}\right)\right| \leq\left|\frac{\hat{p}_{a, b}-p_{a, b}}{p_{a, b}}\right| \\
& \leq\left|\frac{1}{p_{a, b}} \frac{\hat{N}_{\hat{\mathcal{V}}_{a}^{[t]}, \hat{\mathcal{V}}_{b}^{[t]}}}{\hat{N}_{\hat{\mathcal{V}}_{a}^{[t]}, \mathcal{V}}}-1\right|=\left|\frac{1}{p_{a, b}} \frac{N_{\hat{\mathcal{V}}_{a}^{[t]}, \hat{\mathcal{V}}_{b}^{[t]}}}{N_{\hat{\mathcal{V}}_{a}^{[t]}, \mathcal{V}}} \frac{\hat{N}_{\hat{\mathcal{V}}_{a}^{[t]}, \hat{\mathcal{V}}_{b}^{[t]}}}{N_{\hat{\mathcal{V}}_{a}^{[t]}, \hat{\mathcal{V}}_{b}^{[t]}}} \frac{N_{\hat{\mathcal{V}}_{a}^{[t]}, \mathcal{V}}}{\hat{N}_{\hat{\mathcal{V}}_{a}^{[t]}, \mathcal{V}}}-1\right| \\
& \leq\left|\frac{N_{\hat{\mathcal{V}}_{a}^{[t]}, \hat{\mathcal{V}}_{b}^{[t]}}}{N_{\mathcal{V}_{a}, \mathcal{V}_{b}}} \frac{N_{\mathcal{V}_{a}, \mathcal{V}}}{N_{\hat{\mathcal{V}}_{a}^{[t]}, \mathcal{V}}} \frac{\hat{N}_{\hat{\mathcal{V}}_{a}^{[t]}, \hat{\mathcal{V}}_{b}^{[t]}}}{N_{\hat{\mathcal{V}}_{a}^{[t]}, \hat{\mathcal{V}}_{b}^{[t]}}} \frac{N_{\hat{\mathcal{V}}_{a}^{[t]}, \mathcal{V}}}{\hat{N}_{\hat{\mathcal{V}}_{a}^{[t]}, \mathcal{V}}}-1\right|=O_{\mathbb{P}}\left(\frac{e_{n}^{[t]}}{n}+\sqrt{\frac{n}{T}}\right),
\end{aligned}
$$

where the last equality is obtained from the following observations: the four ratios involved in the last inequality are all close to 1 . The two first ratios

imsart-aos ver. 2014/10/16 file: JS_AP_SY__CBMC_-arXiv.tex date: July 31, 2019 
capture the error due to the fact that our estimated clusters are not the true clusters, i.e., $\mathcal{V}_{a}^{[t]} \neq \mathcal{V}_{a}$. However, by assumption, we have $\mid N_{\hat{\mathcal{V}}_{a}^{[t]}, \hat{\mathcal{V}}_{b}^{[t]}} / N_{\mathcal{V}_{a}, \mathcal{V}_{b}}-$ $1 \mid=O_{\mathbb{P}}\left(e_{n}^{[t]} / n\right)$. The same inequality holds for the second ratio. To control the two last ratios, we use the concentration result (45) (this result is uniform over

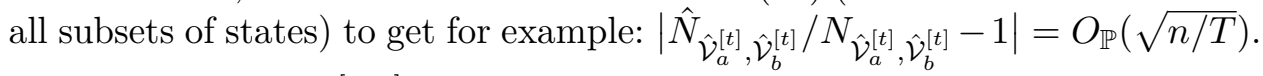

Next, since $x \in \mathcal{E}_{\mathcal{H}}^{[t+1]}$ implies that $x \in \Gamma$, it follows from (44) that

$$
\hat{N}_{x, \mathcal{V}}+\hat{N}_{\mathcal{V}, x}=O\left(\frac{T}{n} \ln \frac{T}{n}\right) .
$$

with high probability. Then from (167) and (168) it follows that

$$
\left|E_{3}\right|=O_{\mathbb{P}}\left(e_{n}^{[t+1]} \sqrt{\frac{T}{n}} \ln \frac{T}{n}+e_{n}^{[t+1]} \frac{e_{n}^{[t]}}{n} \frac{T}{n} \ln \frac{T}{n}\right) .
$$

This completes the proof.

LEMma 15. We have $\left|E_{4}\right|=O_{\mathbb{P}}\left(\sqrt{\frac{T}{n}} e_{n}^{[t+1]}+\frac{e_{n}^{[t]}}{n} \frac{T}{n} e_{n}^{[t+1]}\right)$.

Proof. Let $k \in\{1, \ldots, K\}$ to examine any one of the summands in $E_{4}$. We (i) center and use the triangle inequality to bound all summands as

$$
\begin{aligned}
\left|\frac{\hat{N}_{\hat{\mathcal{V}}_{k}^{[t]}, \mathcal{V}}}{\left|\hat{\mathcal{V}}_{k}^{[t]}\right|}-\frac{\hat{N}_{\mathcal{V}_{k}, \mathcal{V}}}{\left|\mathcal{V}_{k}\right|}\right| \stackrel{(\mathrm{i})}{\leq} \mid \frac{\hat{N}_{\hat{\mathcal{V}}_{k}^{[t]}, \mathcal{V}}-N_{\hat{\mathcal{V}}_{k}^{[t]}, \mathcal{V}}|+| \frac{\hat{N}_{\mathcal{V}_{k}, \mathcal{V}}-N_{\mathcal{V}_{k}, \mathcal{V}}}{\left|\mathcal{V}_{k}\right|} \mid}{\left|\hat{\mathcal{V}}_{k}\right|} & +\left|\frac{N_{\hat{\mathcal{V}}_{k}^{[t]}, \mathcal{V}}}{\left|\hat{\mathcal{V}}_{k}^{[t]}\right|}-\frac{N_{\mathcal{V}_{k}, \mathcal{V}}}{\left|\mathcal{V}_{k}\right|}\right|=O_{\mathbb{P}}\left(\sqrt{\frac{T}{n}}+\frac{e_{n}^{[t]}}{n} \frac{T}{n}\right)
\end{aligned}
$$

where the last equality is obtained the same way as in the proof of Lemma 14 (i.e., from (45)). Thus, $\left|E_{4}\right|=O_{\mathbb{P}}\left(\sqrt{T / n} e_{n}^{[t+1]}+\frac{e_{n}^{[t]}}{n} \frac{T}{n} e_{n}^{[t+1]}\right)$.

\section{SM6. Supporting propositions.}

SM6.1. Properties of uniform vertex selection.

LEMMA 16. If a state $V^{*}$ is selected uniformly at random from two specific clusters $a, b \in\{1, \ldots, K\}, a \neq b$, and a state $V$ is selected uniformly at random from all states,

$$
\mathbb{P}_{\Phi}\left[V^{*} \in \mathcal{E}\right]=\mathbb{P}_{\Phi}\left[V \in \mathcal{E} \mid V \in \mathcal{V}_{a} \cup \mathcal{V}_{b}\right]
$$

imsart-aos ver. 2014/10/16 file: JS_AP_SY__CBMC__arXiv.tex date: July 31, 2019 
Proof. We have:

$$
\begin{aligned}
\mathbb{P}_{\Phi}\left[V^{*} \in \mathcal{E}\right] & =\sum_{v \in \mathcal{V}_{a} \cup \mathcal{V}_{b}} \mathbb{P}_{\Phi}\left[V^{*} \in \mathcal{E} \mid V^{*}=v\right] \mathbb{P}_{\Phi}\left[V^{*}=v\right] \\
& =\frac{1}{\left|\mathcal{V}_{a}\right|+\left|\mathcal{V}_{b}\right|} \sum_{v \in \mathcal{V}_{a} \cup \mathcal{V}_{b}} \mathbb{P}_{\Phi}[v \in \mathcal{E}]
\end{aligned}
$$

and

$$
\begin{aligned}
& \mathbb{P}_{\Phi}\left[V \in \mathcal{E} \mid V \in \mathcal{V}_{a} \cup \mathcal{V}_{b}\right]=\frac{\sum_{v \in \mathcal{V}} \mathbb{P}_{\Phi}\left[V \in \mathcal{E}, V \in \mathcal{V}_{a} \cup \mathcal{V}_{b} \mid V=v\right] \mathbb{P}_{\Phi}[V=v]}{\mathbb{P}_{\Phi}\left[V \in \mathcal{V}_{a} \cup \mathcal{V}_{b}\right]} \\
& \quad=\frac{\sum_{v \in \mathcal{V}_{a} \cup \mathcal{V}_{b}} \mathbb{P}_{\Phi}[v \in \mathcal{E}] /|\mathcal{V}|}{\left(\left|\mathcal{V}_{a}\right|+\left|\mathcal{V}_{b}\right|\right) /|\mathcal{V}|}=\frac{1}{\left|\mathcal{V}_{a}\right|+\left|\mathcal{V}_{b}\right|} \sum_{v \in \mathcal{V}_{a} \cup \mathcal{V}_{b}} \mathbb{P}_{\Phi}[v \in \mathcal{E}] .
\end{aligned}
$$

The lemma follows.

LEMma 17. If a state $V$ is selected uniformly at random from all states, then $\mathbb{E}_{\Phi}[|\mathcal{E}|]=n \mathbb{P}_{\Phi}[V \in \mathcal{E}]$.

Proof. We have:

$$
\mathbb{E}_{\Phi}[|\mathcal{E}|]=\mathbb{E}_{\Phi}\left[\sum_{v \in \mathcal{V}} \mathbb{1}[v \in \mathcal{E}]\right]=\sum_{v \in \mathcal{V}} \mathbb{E}_{\Phi}[\mathbb{1}[v \in \mathcal{E}]]=\sum_{v \in \mathcal{V}} \mathbb{P}_{\Phi}[v \in \mathcal{E}]
$$

and

$$
\begin{aligned}
n \mathbb{P}_{\Phi}[V \in \mathcal{E}] & =n \sum_{v \in \mathcal{V}} \mathbb{P}_{\Phi}[V \in \mathcal{E} \mid V=v] \mathbb{P}_{\Phi}[V=v] \\
& =n \sum_{v \in \mathcal{V}} \mathbb{P}_{\Phi}[v \in \mathcal{E}] \frac{1}{|\mathcal{V}|}=\sum_{v \in \mathcal{V}} \mathbb{P}_{\Phi}[v \in \mathcal{E}]
\end{aligned}
$$

which completes the proof.

SM6.2. Asymptotic comparisons between $P$ and $Q$ 's entries. Recall that $R_{x, y}=Q_{x, y} / P_{x, y}$ for $x, y \in \mathcal{V}$.

Lemma 18. The following properties hold:

(i) $R_{x, y}=1+n^{-1}\left(\mathbb{1}\left[\sigma(y)=\sigma\left(V^{*}\right)\right] / \alpha_{\sigma(y)}-q_{\sigma(x), 0} /\left(p_{\sigma(x), \sigma(y)} K\right)\right)+O\left(n^{-2}\right)$ for $x, y \neq V^{*}$,

(ii) $R_{x, V^{*}}=q_{\omega(x), 0} \alpha_{\sigma\left(V^{*}\right)} / p_{\omega(x), \sigma\left(V^{*}\right)}+O\left(n^{-1}\right)$ for $x \in \mathcal{V} \backslash\left\{V^{*}\right\}$,

(iii) $R_{V^{*}, y}=q_{0, \omega(x)} / p_{\sigma\left(V^{*}\right), \omega(x)}+O\left(n^{-1}\right)$ for $y \in \mathcal{V} \backslash\left\{V^{*}\right\}$.

imsart-aos ver. 2014/10/16 file: JS_AP_SY__CBMC__arXiv.tex date: July 31, 2019 
Proof. Let $x, y \in \mathcal{V} \backslash\left\{V^{*}\right\}$. Using a Taylor expansion (i), we find that:

$$
R_{x, y} \stackrel{(4,25)}{=} \frac{p_{\sigma(x), \sigma(y)}-\frac{q_{\sigma(x), 0}}{K n}}{p_{\sigma(x), \sigma(y)}} \cdot \frac{\left|\mathcal{V}_{\sigma(y)}\right|-\mathbb{1}[\sigma(x)=\sigma(y)]}{\left|\mathcal{V}_{\sigma(y)}\right|-\mathbb{1}\left[\sigma(y)=\sigma\left(V^{*}\right)\right]-\mathbb{1}[\sigma(x)=\sigma(y)]}
$$

$$
\stackrel{(\mathrm{i})}{=} 1+\frac{1}{n}\left(\frac{\mathbb{1}\left[\sigma(y)=\sigma\left(V^{*}\right)\right]}{\alpha_{\sigma(y)}}-\frac{q_{\sigma(x), 0}}{p_{\sigma(x), \sigma(y)} K}\right)+O\left(\frac{1}{n^{2}}\right) .
$$

Similarly for $x \in \mathcal{V} \backslash\left\{V^{*}\right\}$

$$
R_{x, V^{*}} \stackrel{(4,25)}{=} \frac{q_{\omega(x), 0}}{p_{\sigma(x), \sigma\left(V^{*}\right)}} \cdot \frac{\left|\mathcal{V}_{\sigma\left(V^{*}\right)}\right|-\mathbb{1}\left[\sigma(x)=\sigma\left(V^{*}\right)\right]}{n} \stackrel{(\mathrm{i})}{=} \frac{q_{\omega(x), 0} \alpha_{\sigma\left(V^{*}\right)}}{p_{\sigma(x), \sigma\left(V^{*}\right)}}+O\left(\frac{1}{n}\right),
$$

and for $y \in \mathcal{V} \backslash\left\{V^{*}\right\}$

$$
R_{V^{*}, y} \stackrel{(4,25)}{=} \frac{q_{0, \omega(y)}}{p_{\sigma\left(V^{*}\right), \sigma(y)}} \cdot \frac{\left|\mathcal{V}_{\sigma(y)}\right|-\mathbb{1}\left[\sigma\left(V^{*}\right)=\sigma(y)\right]}{\left|\mathcal{W}_{\omega(y)}\right|} \stackrel{(\mathrm{i})}{=} \frac{q_{0, \omega(y)}}{p_{\sigma\left(V^{*}\right), \sigma(y)}}+O\left(\frac{1}{n}\right) .
$$

This completes the proof.

Recall that $S_{x, y, u, v}=\ln R_{x, y} \cdot \ln R_{u, v}$ for $x, y, u, v \in \mathcal{V}$.

COROLlary 2. The following properties hold:

(i) $S_{x, y, u, v}=O\left(n^{-2}\right)$ if all $x, y, u, v \neq V^{*}$,

(ii) $S_{x, y, u, v}=O\left(n^{-1}\right)$ if one of $x, y, u, v$ is $V^{*}$,

(iii) $S_{x, y, u, v}=O(1)$ if two of $x \neq y, u \neq v$ are $V^{*}$.

PROOF. These properties are all direct consequences of Lemma 18, which can be seen by using the Taylor expansion $\ln (1+x)=x+O\left(x^{2}\right)$ for $x \approx 0$ and expanding the product. Consider for example the case $x, y, u, v \in \mathcal{V} \backslash\left\{V^{*}\right\}$ :

$$
S_{x, y, u, v}=\ln R_{x, y} \cdot \ln R_{u, v}=\ln \left(1+O\left(\frac{1}{n}\right)\right) \cdot \ln \left(1+O\left(\frac{1}{n}\right)\right)=O\left(\frac{1}{n^{2}}\right) .
$$

The remaining cases follow similarly.

SM6.3. The KL-divergence and the log-square expression.

LEMmA 19. When $\sum_{x \in \mathcal{X}} p_{x}=\sum_{x \in \mathcal{X}} q_{x}=1$ and $p_{x} \geq 0$ and $q_{x} \geq 0$ for all $x \in \mathcal{X}, \sum_{x \in \mathcal{X}} p_{x}\left(\ln \frac{p_{x}}{q_{x}}\right)^{2} \leq 2\left(\max _{x} \frac{p_{x} \vee q_{x}}{p_{x} \wedge q_{x}}\right)^{2} \mathrm{KL}(p \| q)$. 
Proof. Let $\mathcal{X}_{+} \triangleq\left\{x \in \mathcal{X}: p_{x}>q_{x}\right\}$ and $\mathcal{X}_{-} \triangleq\left\{x \in \mathcal{X}: p_{x}<q_{x}\right\}$. For any given $x \in \mathcal{X}_{+}$and $x^{\prime} \in \mathcal{X}_{-}$, for all $p_{x}>a>q_{x}$ and $p_{x^{\prime}}<b<q_{x^{\prime}}$

$$
\begin{aligned}
& \frac{d}{d \varepsilon}\left((a+\varepsilon) \ln \frac{a+\varepsilon}{q_{x}}+\right.\left.(b-\varepsilon) \ln \frac{b-\varepsilon}{q_{x^{\prime}}}\right)\left.\right|_{\varepsilon=0}=\ln \frac{a}{q_{x}}-\ln \frac{b}{q_{x^{\prime}}} \\
& \geq \frac{a-q_{x}}{a}+\frac{q_{x^{\prime}}-b}{q_{x^{\prime}}} \geq \frac{a-q_{x}}{p_{x} \vee q_{x}}+\frac{q_{x^{\prime}}-b}{p_{x^{\prime}} \vee q_{x^{\prime}}}
\end{aligned}
$$

Therefore,

$$
\mathrm{KL}(p \| q)=\sum_{x \in \mathcal{X}} p_{x} \ln \frac{p_{x}}{q_{x}} \geq \frac{1}{2} \sum_{x \in \mathcal{X}} \frac{\left(p_{x}-q_{x}\right)^{2}}{p_{x} \vee q_{x}} .
$$

Then, we have

$$
\begin{aligned}
& \sum_{x \in \mathcal{X}} p_{x}\left(\ln \frac{p_{x}}{q_{x}}\right)^{2} \leq \sum_{x \in \mathcal{X}} p_{x} \frac{\left(p_{x}-q_{x}\right)^{2}}{\left(p_{x} \wedge q_{x}\right)^{2}} \\
& \quad \leq \sum_{x \in \mathcal{X}}\left(\max _{x} \frac{p_{x} \vee q_{x}}{p_{x} \wedge q_{x}}\right)^{2} \frac{\left(p_{x}-q_{x}\right)^{2}}{\left(p_{x} \vee q_{x}\right)} \stackrel{(\mathrm{i})}{\leq} 2\left(\max _{x} \frac{p_{x} \vee q_{x}}{p_{x} \wedge q_{x}}\right)^{2} \mathrm{KL}(p \| q),
\end{aligned}
$$

where the last inequality marked (i) stems from (181).

SM6.4. Spectral norm bound for sums of elements of matrices.

Lemma 20. For any matrix $B \in \mathbb{R}^{n \times n}$ and subsets $\mathcal{A}, \mathcal{C} \subseteq\{1, \ldots, n\}$, we have $\sum_{r \in \mathcal{A}} \sum_{c \in \mathcal{C}} B_{r c}=1_{\mathcal{A}}^{\mathrm{T}} B 1_{\mathcal{C}}$. Furthermore, $1_{\mathcal{A}}^{\mathrm{T}} B 1_{\mathcal{C}} \leq\|B\| \sqrt{|\mathcal{A}||\mathcal{C}|}$.

Proof. We have:

$$
\begin{aligned}
1_{\mathcal{A}}^{\mathrm{T}} B 1_{\mathcal{C}} & =1_{\mathcal{A}} \mathrm{T}\left(\sum_{r=1}^{n}\left(\sum_{c=1}^{n} B_{r c} \mathbb{1}[c \in \mathcal{C}] e_{n, r}\right)\right) \\
& =\sum_{c^{\prime}=1}^{n} \mathbb{1}\left[c^{\prime} \in \mathcal{A}\right] e_{n, c^{\prime}} \mathrm{T}\left(\sum_{r=1}^{n}\left(\sum_{c \in \mathcal{C}} B_{r c} e_{n, r}\right)\right)=\sum_{c^{\prime} \in \mathcal{A}} \sum_{r=1}^{n} \sum_{c \in \mathcal{C}} B_{r c} e_{n, c^{\prime}} \mathrm{T}_{e, r} \\
& =\sum_{c^{\prime} \in \mathcal{A}} \sum_{r=1}^{n} \sum_{c \in \mathcal{C}} B_{r c} \mathbb{1}\left[c^{\prime}=r\right]=\sum_{r \in \mathcal{A}} \sum_{c \in \mathcal{C}} B_{r c},
\end{aligned}
$$

which proves the first statement.

For the second statement, first note that (i) $1_{\mathcal{A}}{ }^{\mathrm{T}} B 1_{\mathcal{C}} \in \mathbb{R}$ and therefore $1_{\mathcal{A}}^{\mathrm{T}} B 1_{\mathcal{C}} \leq\left|1_{\mathcal{A}}^{\mathrm{T}} B 1_{\mathcal{C}}\right|$. By (ii) applying the Cauchy-Schwarz inequality twice, and (iii) the consistency of subordinate norms, we obtain

$$
1_{\mathcal{A}}^{\mathrm{T}} B 1_{\mathcal{C}} \stackrel{(\mathrm{i})}{\leq}\left|1_{\mathcal{A}}^{\mathrm{T}} B 1_{\mathcal{C}}\right| \stackrel{\text { (ii) }}{\leq}\left\|1_{\mathcal{A}}\right\|_{2}\left\|B 1_{\mathcal{C}}\right\|_{2} \stackrel{\text { (iii) }}{\leq}\left\|1_{\mathcal{A}}\right\|_{2}\|B\|\left\|1_{\mathcal{C}}\right\|_{2} .
$$

imsart-aos ver. 2014/10/16 file: JS_AP_SY__CBMC__arXiv.tex date: July 31, 2019 
Lastly for any set $\mathcal{A} \subseteq\{1, \ldots, n\}$, we have that $1_{\mathcal{A}} \in\{0,1\}^{n}$, and therefore $\left\|1_{\mathcal{A}}\right\|_{2}=\sqrt{\left\|1_{\mathcal{A}}\right\|_{1}}=\sqrt{|\mathcal{A}|}$. Applying this bound for the sets $\mathcal{A}, \mathcal{C}$ concludes the proof.

SM6.5. Stochastic boundedness properties. Recall that when we write $X_{n}=O_{\mathbb{P}}\left(a_{n}\right)$ for a sequence of random variables $\left\{X_{n}\right\}_{n=1}^{\infty}$ and some deterministic sequence $\left\{a_{n}\right\}_{n=1}^{\infty}$, this is equivalent to saying

$$
\forall_{\varepsilon>0} \exists_{\delta_{\varepsilon}, N_{\varepsilon}}: \mathbb{P}\left[\left|\frac{X_{n}}{a_{n}}\right| \geq \delta_{\varepsilon}\right] \leq \varepsilon \forall_{n>N_{\varepsilon}}
$$

Lemma 21. Let $\cup_{n=1}^{\infty}\left\{X_{n}\right\}_{n \geq 0}, \cup_{n=1}^{\infty}\left\{Y_{n}\right\}$ denote two families of random variables with the properties that $X_{n}, Y_{n} \geq 0, X_{n}=O_{\mathbb{P}}\left(x_{n}\right)$, and $Y_{n}=O_{\mathbb{P}}\left(y_{n}\right)$, where $\left\{x_{n}\right\}_{n=1}^{\infty},\left\{y_{n}\right\}_{n=1}^{\infty}$ denote two deterministic sequences with $x_{n}, y_{n} \in$ $[0, \infty)$. Then $X_{n} Y_{n}=O_{\mathbb{P}}\left(x_{n} y_{n}\right)$. Similarly if $X_{n}=\Omega_{\mathbb{P}}\left(x_{n}\right), Y_{n}=\Omega_{\mathbb{P}}\left(y_{n}\right)$, then $X_{n} Y_{n}=\Omega_{\mathbb{P}}\left(x_{n} y_{n}\right)$.

Proof. Let $\varepsilon>0$. Choose $\delta_{\varepsilon}^{X}, N_{\varepsilon}^{X}$ and $\delta_{\varepsilon}^{Y}, N_{\varepsilon}^{Y}$ such that $\mathbb{P}\left[X_{n} \geq \delta_{\varepsilon}^{X} x_{n}\right] \leq$ $\varepsilon / 3$ for $n>N_{\varepsilon}^{X}$ and $\mathbb{P}\left[Y_{n} \geq \delta_{\varepsilon}^{Y} y_{n}\right] \leq \varepsilon / 3$ for $n>N_{\varepsilon}^{Y}$. Pick any $\delta_{\varepsilon}>\delta_{\varepsilon}^{X} \delta_{\varepsilon}^{Y}$. With these choices,

$$
\begin{aligned}
\mathbb{P}\left[\left|\frac{X_{n} Y_{n}}{x_{n} y_{n}}\right| \geq \delta_{\varepsilon}\right]= & \mathbb{P}\left[\left|\frac{X_{n} Y_{n}}{x_{n} y_{n}}\right| \geq \delta_{\varepsilon}, X_{n} \geq \delta_{\varepsilon}^{X} x_{n}, Y_{n} \geq \delta_{\varepsilon}^{Y} y_{n}\right] \\
& +\mathbb{P}\left[\left|\frac{X_{n} Y_{n}}{x_{n} y_{n}}\right| \geq \delta_{\varepsilon}, X_{n} \geq \delta_{\varepsilon}^{X} x_{n}, Y_{n}<\delta_{\varepsilon}^{Y} y_{n}\right] \\
& +\mathbb{P}\left[\left|\frac{X_{n} Y_{n}}{x_{n} y_{n}}\right| \geq \delta_{\varepsilon}, X_{n}<\delta_{\varepsilon}^{X} x_{n}, Y_{n} \geq \delta_{\varepsilon}^{Y} y_{n}\right] \\
& +\mathbb{P}\left[\left|\frac{X_{n} Y_{n}}{x_{n} y_{n}}\right| \geq \delta_{\varepsilon}, X_{n}<\delta_{\varepsilon}^{X} x_{n}, Y_{n}<\delta_{\varepsilon}^{Y} y_{n}\right] \leq \varepsilon .
\end{aligned}
$$

We have shown that

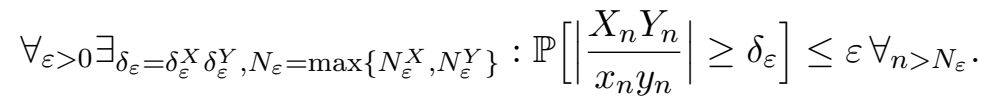

This completes the proof.

The following lemma can be proved similarly:

Lemma 22. Let $\cup_{n=1}^{\infty}\left\{X_{n}\right\}_{n \geq 0}, \cup_{n=1}^{\infty}\left\{Y_{n}\right\}$ denote two families of random variables with the properties that $X_{n}, Y_{n} \geq 0, X_{n} / Y_{n}=\Omega_{\mathbb{P}}\left(y_{n}\right)$, and $X_{n}=$ $O_{\mathbb{P}}\left(x_{n}\right)$, where $\left\{x_{n}\right\}_{n=1}^{\infty},\left\{y_{n}\right\}_{n=1}^{\infty}$ denote two deterministic sequences with $x_{n}, y_{n} \in[0, \infty)$. Then $Y_{n}=O_{\mathbb{P}}\left(x_{n} / y_{n}\right)$.

imsart-aos ver. 2014/10/16 file: JS_AP_SY__CBMC_-arXiv.tex date: July 31, 2019 
Lemma 23. Let $\left\{s_{n}\right\}_{n=1}^{\infty}$ denote a deterministic sequence with $s_{n} \in$ $\mathbb{N}_{+}$. Let $\cup_{n=1}^{\infty} \cup_{m=1}^{s_{n}}\left\{X_{m, n}\right\}$ denote a family of random variables with the properties that $X_{m, n} \geq 0$, and $\exists_{\delta, N}: \mathbb{E}\left[X_{m, n}\right] \leq \delta x_{n} \forall_{m=1, \ldots, s_{n}} \forall_{n>N}$. Then $S_{n}=\sum_{m=1}^{s_{n}} X_{m, n}=O_{\mathbb{P}}\left(s_{n} x_{n}\right)$.

Proof. Let $\varepsilon>0, \delta_{\varepsilon}^{\Sigma}>0$. Since (i) $X_{m, n}>0$ for all $m, n$, by (ii) Markov's inequality

$$
\mathbb{P}\left[\left|\frac{S_{n}}{s_{n} x_{n}}\right| \geq \delta_{\varepsilon}^{\Sigma}\right] \stackrel{(\mathrm{i})}{=} \mathbb{P}\left[\frac{1}{s_{n} x_{n}} \sum_{m=1}^{s_{n}} X_{m, n} \geq \delta_{\varepsilon}^{\Sigma}\right] \stackrel{(\mathrm{ii})}{\leq} \frac{\sum_{m=1}^{s_{n}} \mathbb{E}\left[X_{m, n}\right]}{\delta_{\varepsilon}^{\Sigma} s_{n} x_{n}} .
$$

By assumption $\exists_{\delta, N}: \mathbb{E}\left[X_{m, n}\right] \leq \delta x_{n} \forall_{m=1, \ldots, s_{n}} \forall_{n>N}$. Choose $\delta, N$ as such. Specify $\delta_{\varepsilon}^{\Sigma}=\delta / \varepsilon$. By (187), we have thus shown that

$$
\forall_{\varepsilon>0} \exists_{\delta_{\varepsilon}^{\Sigma}=\delta / \varepsilon, N_{\varepsilon}=N}: \mathbb{P}\left[\left|\frac{S_{n}}{s_{n} x_{n}}\right| \geq \delta_{\varepsilon}^{\Sigma}\right] \leq \varepsilon \forall_{n>N_{\varepsilon}} .
$$

Equivalently, $S_{n}=O_{\mathbb{P}}\left(s_{n} x_{n}\right)$. This completes the proof.

Lemma 24. Let $\cup_{n=1}^{\infty} \cup_{m=1}^{n}\left\{X_{m, n}\right\}$ denote a family of random variables with the properties that $X_{m, n} \geq 0$, and $\exists_{\delta, N}: \mathbb{E}\left[X_{m, n}\right] \leq \delta x_{n} \forall_{m=1, \ldots, n} \forall_{n>N}$. If $\left\{Y_{n}\right\}_{n=1}^{\infty}$ is a sequence of random variables with the properties that $Y_{n} \in$ $\{1, \ldots, n\}$, and $Y_{n}=O_{\mathbb{P}}\left(y_{n}\right)$ for some deterministic sequence $\left\{y_{n}\right\}_{n=1}^{\infty}$ with $y_{n} \in \mathbb{N}_{+}$, then $Z_{n}=\sum_{m=1}^{Y_{n} \wedge n} X_{m, n}=O_{\mathbb{P}}\left(\left(y_{n} \wedge n\right) x_{n}\right)$.

Proof. Let $\varepsilon>0, \delta_{\varepsilon}^{Z}>0$. Then

$$
\begin{aligned}
\mathbb{P}\left[\left|\frac{Z_{n}}{y_{n} x_{n}}\right| \geq \delta_{\varepsilon}^{Z}\right] & =\mathbb{P}\left[\left|\frac{Z_{n}}{y_{n} x_{n}}\right| \geq \delta_{\varepsilon}^{Z},\left|\frac{Y_{n}}{y_{n}}\right| \geq \delta_{\varepsilon}^{Y}\right]+\mathbb{P}\left[\left|\frac{Z_{n}}{y_{n} x_{n}}\right| \geq \delta_{\varepsilon}^{Z},\left|\frac{Y_{n}}{y_{n}}\right|<\delta_{\varepsilon}^{Y}\right] \\
& (189) \quad \leq \mathbb{P}\left[\left|\frac{Y_{n}}{y_{n}}\right| \geq \delta_{\varepsilon}^{Y}\right]+\mathbb{P}\left[\left|\frac{1}{y_{n} x_{n}} \sum_{m=1}^{\left(\delta_{\varepsilon}^{Y} y_{n}\right) \wedge n} X_{m, n}\right| \geq \delta_{\varepsilon}^{Z}\right] .
\end{aligned}
$$

By assumption $Y_{n}=O_{\mathbb{P}}\left(y_{n}\right)$, so we can choose $\delta_{\varepsilon}^{Y} \in \mathbb{N}_{+}, N_{\varepsilon}^{Y}>0$ such that $\mathbb{P}\left[\left|Y_{n} / y_{n}\right| \geq \delta_{\varepsilon}^{Y}\right] \leq \varepsilon / 2$ for all $n>N_{\varepsilon}^{Y}$. Write $\delta_{\varepsilon}^{Z}=\delta_{\varepsilon}^{Y} \delta_{\varepsilon}^{\Sigma}$, and we will specify $\delta_{\varepsilon}^{\Sigma}$ in a moment. Presently, we are at

$$
\begin{aligned}
\mathbb{P}\left[\left|\frac{Z_{n}}{y_{n} x_{n}}\right| \geq \delta_{\varepsilon}^{Z}\right] & \leq \frac{\varepsilon}{2}+\mathbb{P}\left[\left|\frac{1}{\left(\delta_{\varepsilon}^{Y} y_{n}\right) x_{n}} \sum_{m=1}^{\left(\delta_{\varepsilon}^{Y} y_{n}\right) \wedge n} X_{m, n}\right| \geq \delta_{\varepsilon}^{\Sigma}\right] \\
& \leq \frac{\varepsilon}{2}+\mathbb{P}\left[\left|\frac{1}{\left(\delta_{\varepsilon}^{Y} y_{n} \wedge n\right) x_{n}} \sum_{m=1}^{\delta_{\varepsilon}^{Y} y_{n} \wedge n} X_{m, n}\right| \geq \delta_{\varepsilon}^{\Sigma}\right] .
\end{aligned}
$$

imsart-aos ver. 2014/10/16 file: JS_AP_SY__CBMC_-arXiv.tex date: July 31, 2019 
The assumptions on the family $\left\{X_{m, n}\right\}_{m, n=1}^{\infty}$ now allow us to apply Lemma 23: specifically, there exist $\delta_{\varepsilon}^{\Sigma}, N_{\varepsilon}^{\Sigma}$ such that the final term is bounded by $\varepsilon / 2$ for all $n>N_{\varepsilon}^{\Sigma}$. Summarizing, we have shown that

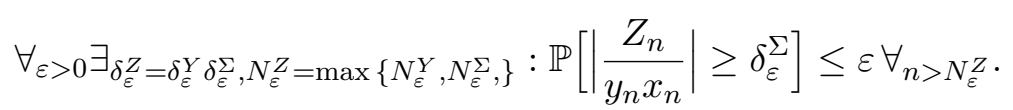

Equivalently, $Z_{n}=O_{\mathbb{P}}\left(y_{n} x_{n}\right)$.

Lemma 25. Let $\cup_{n=1}^{\infty}\left\{X_{n}\right\}_{n \geq 0}, \cup_{n=1}^{\infty}\left\{Y_{n}\right\}$ denote two families of random variables with the properties that $\mathbb{P}\left[X_{n} \leq Y_{n}\right]=1, X_{n}=\Omega_{\mathbb{P}}\left(x_{n}\right)$, and $Y_{n}=O_{\mathbb{P}}\left(y_{n}\right)$, where $\left\{x_{n}\right\}_{n=1}^{\infty},\left\{y_{n}\right\}_{n=1}^{\infty}$ denote two deterministic sequences with $x_{n}, y_{n} \in \mathbb{R}$. Then, $x_{n}=O\left(y_{n}\right)$.

Proof. We prove the result by contradiction. Recall first that the assumptions imply that for every $\varepsilon^{X}, \varepsilon^{Y}>0$, there exist $\delta_{\varepsilon}^{X}, \delta_{\varepsilon}^{Y}>0$ such that

$$
\lim _{n \rightarrow \infty} \mathbb{P}\left[X_{n} \leq \delta_{\varepsilon}^{X} x_{n}\right] \leq \varepsilon^{X}, \quad \lim _{n \rightarrow \infty} \mathbb{P}\left[Y_{n} \geq \delta_{\varepsilon}^{Y} y_{n}\right] \leq \varepsilon^{Y} .
$$

Also note that by (i) definition of conditional probability, (ii) the De Morgan laws, and (iii) $\mathbb{P}\left[\left\{X_{n} \leq \delta^{X} x_{n}\right\} \cap\left\{Y_{n} \geq \delta^{Y} y_{n}\right\}\right] \geq 0$, it follows that

$$
\begin{aligned}
& 0= \mathbb{P}\left[X_{n}>Y_{n}\right] \geq \mathbb{P}\left[\left\{X_{n}>Y_{n}\right\} \cap\left\{X_{n}>\delta^{X} x_{n}\right\} \cap\left\{Y_{n}<\delta^{Y} y_{n}\right\}\right] \\
& \stackrel{(\mathrm{i})}{=} \mathbb{P}\left[X_{n}>Y_{n} \mid\left\{X_{n}>\delta^{X} x_{n}\right\} \cap\left\{Y_{n}<\delta^{Y} y_{n}\right\}\right] \times \cdots \\
& \quad \times\left(1-\mathbb{P}\left[\left(\left\{X_{n}>\delta^{X} x_{n}\right\} \cap\left\{Y_{n}<\delta^{Y} y_{n}\right\}\right)^{\mathrm{c}}\right]\right) \\
& \stackrel{(\text { ii) }}{=} \mathbb{P}\left[X_{n}>Y_{n} \mid\left\{X_{n}>\delta^{X} x_{n}\right\} \cap\left\{Y_{n}<\delta^{Y} y_{n}\right\}\right] \times \cdots \\
& \quad \times\left(1-\mathbb{P}\left[\left\{X_{n} \leq \delta^{X} x_{n}\right\} \cup\left\{Y_{n} \geq \delta^{Y} y_{n}\right\}\right]\right) \\
& \stackrel{(\text { iii) }}{\geq} \mathbb{P}\left[X_{n}>Y_{n} \mid\left\{X_{n}>\delta^{X} x_{n}\right\} \cap\left\{Y_{n}<\delta^{Y} y_{n}\right\}\right] \times \cdots \\
& \quad \times\left(1-\mathbb{P}\left[\left\{X_{n} \leq \delta^{X} x_{n}\right\}\right]-\mathbb{P}\left[\left\{Y_{n} \geq \delta^{Y} y_{n}\right\}\right]\right) .
\end{aligned}
$$

Now suppose that $x_{n}=\omega\left(y_{n}\right)$. By then taking the limit $n \rightarrow \infty$ both left and right, we obtain the inequality $0 \geq 1-\varepsilon^{X}-\varepsilon^{Y}$, which is a contradiction. Hence it must be that $x_{n}=O\left(y_{n}\right)$.

(This concludes the supplementary material).

imsart-aos ver. 2014/10/16 file: JS_AP_SY__CBMC__arXiv.tex date: July 31, 2019 
KTH Royal Institute of Technology

School of Electrical Engineering

Dept. of Automatic Control

OSQULdASVÄG 10, Stockholm 10044, Sweden

E-MAIL: alepro@kth.se

Delft University of Technology

Faculty of Electrical Engineering, Mathematics \& Computer Science Dept. of Quantum \& Computer Engineering

Mekelweg 4, 2628CD Delft, The Netherlands

E-MAIL: j.sanders@tudelft.nl

Korea Advanced Institute of Science and Technology

Dept. of Industrial \& Systems EngineERING

291 Daehak-ro, Eoeun-dong, Yuseong-Gu, South Korea

E-MAIL: yunseyoung@kaist.ac.kr 